\title{
Crystal Structures of a Bacterial Isocitrate Dehydrogenase and the Human Sulfamidase:
}

\section{Pushing the Limits of Molecular Replacement}

\author{
Dissertation zur Erlangung des \\ mathematisch-naturwissenschaftlichen Doktorgrades \\ "Doctor rerum naturalium" \\ der Georg-August-Universität Göttingen \\ im Promotionsprogramm Chemie der \\ Georg-August University School of Science (GAUSS)
}

vorgelegt von

Navdeep Singh Sidhu

aus Daudhar, Indien

Göttingen, 2013 


\section{Betreuungsausschuss}

Prof. Dr. George M. Sheldrick, FRS, Institut für Anorganische Chemie

Prof. Dr. Ralf Ficner, Institut für Mikrobiologie und Genetik

\section{Mitglieder der Prüfungskommission}

Referent: Prof. Dr. George M. Sheldrick

Koreferent: Prof. Dr. Ralf Ficner

weitere Mitglieder

Prof. Dr. Kai Tittmann

Prof. Dr. Hartmut Laatsch

Priv. Doz. Dr. Birger Dittrich

Dr. Francesca Fabbiani

Tag der mündlichen Prüfung: 09.01.2014 


"If, in some cataclysm, all of scientific knowledge were to be destroyed, and only one sentence passed on to the next generation of creatures, what statement would contain the most information in the fewest words? I believe it is the atomic hypothesis (or the atomic fact, or whatever you wish to call it) that all things are made up of atoms-little particles that move around in perpetual motion, attracting each other when they are a little distance apart, but repelling upon being squeezed into one another. In that one sentence [...] there is an enormous amount of information about the world, if just a little imagination and thinking are applied."

-Richard P. Feynman 



\section{Danksagung}

I am indebted to Prof. George Sheldrick for all his caring mentoring and support. He provided me with an inspiring and stimulating educational environment imbued with the Enlightenment spirit. It has been a great privilege to learn from discussions with him on my research and the many dimensions of crystallography and its practice, including the worlds of large and small molecules. German has just the right word for him, Doktorvater. I am indebted also to Prof. Isabel Usón from the Instituto de Biologia Molecular de Barcelona, Barcelona, Spain. She taught me most of what I know about practical macromolecular structure solution using molecular replacement-after I thought I knew anything about it. Discussions with her were crucial in selecting an optimum strategy for solving the sulfamidase structure, and in designing a search fragment. She ran the search model through ARCIMBOLDO at a computer grid to obtain an initial rotationtranslation search solution. I doubt I would have succeeded in further processing this initial solution and coaxing the result to refine, which proved to be a major task, and in ultimately solving the sulfamidase structure without her invaluable advice and inspiring guidance. I thank George and Isabel for, to borrow a phrase from David Harker, "sparing neither criticism nor encouragement" in their guidance.

I am grateful to Prof. Ralf Ficner for being my Koreferent and for his critique and comments on my work. I thank him and the rest of my thesis committee for generously finding time for me in their busy schedules.

I express my deepest appreciation to Prof. Robert Steinfeld and Dr. Ralph Krätzner of the Department of Neuropediatrics, University of Göttingen for their valuable support and advice during my work on sulfamidase. I have benefitted greatly from discussions with them on many aspects of the project. I also thank Prof. Jutta Gärtner for financial support. Kathrin Schreiber purified sulfamidase with expertise, giving me an excellent starting point for further work on crystallization experiments. Marcel Grapp helped me find my way around instruments and material in the lab.

My sincere thanks go also to Dr. Stefan Becker from the Max Planck Institute for Biophysical Chemistry, Göttingen, for allowing me to use the crystallization robots. I owe my thanks to him and to the late Dr. Kevin Pröpper for teaching me how to use them. Dr. Tim Grüne accompanied me and helped during a lowerresolution data collection trip. Kevin, Stefan and Priv. Doz. Birger Dittrich gave me a generous helping hand by collecting higher-resolution data on some of my crystals, including two datasets that were used to solve the sulfamidase structure. I am grateful to Birger also for teaching me much about data collection and for discussions on many practical aspects of small molecule crystallography. I sincerely thank Dr. Regine Herbst-Irmer for her warm support and for giving me my first 
lessons on handling twinned crystals. Tim has always been highly generous in advising me about macromolecular data processing and in helping me jump through computer software and hardware hoops. Dr. Andrea Thorn got me started with TLS refinement of IDH. I could always rely on her for generous and warm-hearted suggestions. Dr. Christian Grosse guided me through my first small molecule (and twinned) crystal structure and was a great work (and off-work) mate to many of us. Dr. Tobias Beck and Tim gave helpful advice on parallel projects and critique of a draft manuscript. Dr. Christian Hübschle was generous with his advice on ShelXle and was a great desk neighbor besides. Jens Luebben gave me his generous comments on a section of the draft thesis. Claudia Orben was often a source of encouragement in the course of sulfamidase experiments. Dr. Kathrin Meindl helped me get on my feet in PostScript, just as Dr. Julian Holstein encouraged me to get them wet in LATEX. I thank Claudia Millán for her supportive company and discussions. Dr. Daniel Kratzert gave me useful advice on small-molecule data collection. Dalila Griffin always had a beaming smile for us despite all the secretarial work she had to help us with. Helmut Dehnhardt kept the diffractometer running despite all we put it through. I also benefited much from discussions with past Sheldrick group members, especially Drs. Madhumati Sevvana, Marianna Biadene and Honorata Czapinska.

I sincerely thank Prof. Laatsch, Prof. Roesky and Prof. Diederichsen and their groups, especially Drs. Amit Singh, Prinson Samuel and Kartik Mondal for scientific collaborations. I learned much from Dr. Sowa Heidrun's crystallography exercises and from Dr. Francesca Fabbiani and her group's seminar contributions on high-pressure crystallography. Dr. Claudia Stückl and Frau Hampel generously helped with much paperwork. I thank anonymous reviewers of the IDH and sulfamidase papers for their excellent critique and suggestions; and Louise Jones for her generous help with permission to reprint published figures.

I also owe my gratitude to the late Prof. Louis Delbaere, University of Saskatchewan, Saskatoon, Canada, for his expert and warm-hearted guidance on the pre-refinement, and part of refinement, work on isocitrate dehydrogenase. I am indebted to Prof. Bernhard Eikmanns, Ulm University, Ulm, Germany, for so generously shipping me the cloned IDH gene. I thank Fumie Imabayashi for purified IDH and Dr. Sanjukta Aich for practical advice. I have learned much from interactions with Prof. Rob Warrington, who was a constant source of heartwarming inspiration and encouragement. Drs. Pawel Grochulski and Michel Fodje were most generous with help and advice on data collection at the Canadian Light Source. I greatly appreciate helpful discussions with Prof. Hugh Goldie on protein overexpression in bacteria. Yvonne Leduc happily shared many crystallization suggestions. Dr. Lata Prasad gave helpful advice on refinement. Prof. $\mathrm{Yu}$ Luo generously allowed me to learn from his expertise in crystallography. I also express my sincere thanks to Frau Gabriele Schatte for her support and help in 
keeping the in-house diffractometer up and running during many crystal optimization trials. I especially thank Rob, Derek Tannis, Prof. Len Findlay, Prof. David Kaplan and Prof. William Albritton (then Dean, College of Medicine), Gabriele and Yvonne for their kind-hearted support and encouragement following Louis' untimely death. Last, but not least, I thank the aboriginal people of Saskatoon and Saskatchewan, Canada. They taught me so much about what is important in life.

I thank my former teachers, especially Profs. Bob Deschenes, Vladislav Eybl, Jaroslav Racek, Subramanian Ramaswamy and Earle Stellwagen. Rams was also my first X-ray crystallography teacher, who got me excited about this fascinating area of science. I also thank my medical school teachers and former patients in the Czech Republic. I learned much from both of them. My patients partly inspired the pursuit of scientific research to address medically relevant questions.

I am grateful to friends from three continents who minimized-in the mathematical sense-many a burden and maximized many a joy. They often made it possible for me to work with much greater ease than I otherwise would have been able to. I especially remember Achim, Aditya, Agustin, Alejandra, Amrik, Andrew, Anne, Arash, Bhagwant, Bob, Carlos, Christian, Daniela, David, Fanouš, Gurpreet, Honza, Imran, Iveta, Jake, Jasbir, Jitka, Josef, Luboš, Mateen, Navin, Paramjit, Paramjit, Pavan, Pepper, Peter, Petra, Praful, Ray, Rita, Rosie, Rosťa, Stefan, Sukhpal, Sylvia, Tariq, Tracey and Yogi. A German housing manager kind-heartedly rented me a place to stay and later offered to accept payment due only after I got my first paycheck-without asking when that would be. She kept her word despite my telling her. (What a gentle recipe to lower the human cost of a housing bubble-burst.) My heartfelt thanks go to all of them.

Taran, Miranda, Flavia, Candela and Little Tim always lit up Göttingen with their joyous presence every time they visited. Miranda was also my guardian angel. Gurnoor, Kristina, Lucie, Barborka and Katka brought heart-warming smiles with their adorable stories from afar.

I thank Priti, Chiku, Jorge, Barbara, Oma, Julia, Sebastian, Susan, Friedi and Henni, and all of our family members round the world for their continuous love and support.

This work is dedicated to the memory of my father and grandparents; to my mother, arguably my first science teacher; to Anja, with whom I can share so much, for everything; to Kikkar Singh, Taran, Gurnoor, Mio, Miranda and their generation; to my childhood friend Laxman, who loved geometry but was born into poverty; and to the aboriginal singers and dancers of the planet. 



\section{Contents}

Danksagung vii

List of Abbreviations $\quad$ xv

List of Figures $\quad$ xix

List of Tables $\quad$ xxi

\begin{tabular}{ll}
\hline Overview & 1
\end{tabular}

1 Structure determination by X-ray crystallography: A background 3

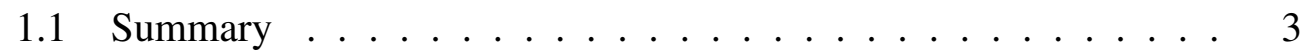

1.1.1 Introduction: The phase problem. . . . . . . . . . . 4

1.1 .2 The Patterson method $\ldots \ldots \ldots$. . . . . . . 6

1.1 .3 Direct methods . . . . . . . . . . . . . . 8

1.2 Macromolecular structure determination . . . . . . . . . . 8

1.2.1 Experimental phasing. . . . . . . . . . . . . . . . 8

1.3 Molecular replacement $\ldots \ldots \ldots$. . . . . . . . . . . . 9

1.3 .1 Rotation search . . . . . . . . . . . . . . . 11

1.3 .2 Translation search . . . . . . . . . . . . . 11

$1.3 .3 \quad$ Bootstrapping the target structure . . . . . . . . . 12

1.4 Density modification $\ldots \ldots \ldots \ldots . \ldots . \ldots 14$

2 Structural study of coenzyme specificity in an isocitrate dehydroge-

nase 17

2.1 Summary . . . . . . . . . . . . . . . . . . . . . 17

2.2 Introduction . . . . . . . . . . . . . . . . . . . 18

2.2.1 Citric acid cycle and isocitrate dehydrogenase . . . . . . 18

$2.2 .2 \quad$ Coenzyme specificity . . . . . . . . . . . . . 19

2.2 .3 Structural studies of coenzyme binding in IDHs . . . . . 20

2.3 Materials and methods . . . . . . . . . . . 20

2.3 .1 Crystallization . . . . . . . . . . . . 20

2.3 .2 Data collection . . . . . . . . . . . . . 22

2.3 .3 Structure solution and refinement . . . . . . . . . . . 22

2.3 .4 Analysis of refined structure . . . . . . . . . . . 23

2.4 Results . . . . . . . . . . . . . . . 23

2.4 .1 Structure solution . . . . . . . . . . . . . 23

2.4 .2 Model quality . . . . . . . . . . . . . . . . . . . . . 24

$2.4 .3 \quad$ Overall structure . . . . . . . . . . . . . . 26

2.4.4 Comparison of CgIDH Holo, Apo1 and Apo2 forms . . . 26 
2.4 .5 Comparison of CgIDH and AvIDH . . . . . . . . . . 29

2.4 .6 The N Hinge and the isocitrate binding site . . . . . . . . . 29

$2.4 .7 \quad \mathrm{NADP}^{+}$binding $\ldots \ldots \ldots \ldots \ldots . \ldots \ldots$

$2.4 .8 \mathrm{Mg}^{2+}$ binding . . . . . . . . . . . . . . . . . . . . . . . . . . . . 34

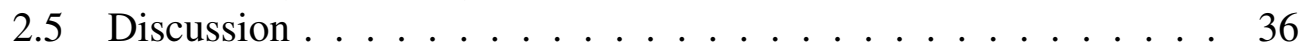

$2.5 .1 \quad$ Cell parameters . . . . . . . . . . . . . . . 36

2.5 .2 Overall structure . . . . . . . . . . . . . . . . . 37

$2.5 .3 \quad$ Hinge bending . . . . . . . . . . . . . . . . 37

$2.5 .4 \quad$ Active site access . . . . . . . . . . . . . . . 38

2.5 .5 Possible regulatory and biological implications . . . . . 39

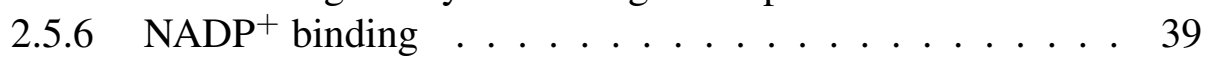

$2.5 .7 \quad \mathrm{NADP}^{+}$specificity . . . . . . . . . . . . . . . . 40

2.5 .8 Nicotinamide binding and disorder. . . . . . . . . . . . 41

2.5 .9 Models for active site access in IDHs . . . . . . . . . . . 43

2.5 .10 Apoenzyme in the asymmetric unit . . . . . . . . . . 43

2.5 .11 Limitations and prospects . . . . . . . . . . . . 45

2.6 Conclusions . . . . . . . . . . . . . . . . . . 45

\begin{tabular}{|lll}
\hline 3 & Crystal structure of human sulfamidase & 47
\end{tabular}

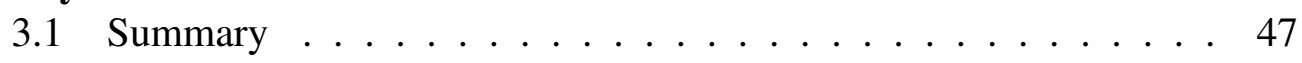

3.2 Introduction . . . . . . . . . . . . . . . . . . . . . . 49

3.3 Materials and Methods . . . . . . . . . . . . . . . . 51

3.3 .1 The sulfamidase gene. . . . . . . . . . . . . 51

3.3 .2 Protein expression and purification . . . . . . . . . . . 52

3.3 .3 Protein crystallization trials $\ldots \ldots \ldots . . \ldots . . \ldots 52$

3.3 .4 Data collection . . . . . . . . . . . . . . 53

3.3 .5 Structure refinement . . . . . . . . . . . . . 54

3.4 Results . . . . . . . . . . . . . . . . . . . 55

3.4.1 Buffer exchange and crystallization . . . . . . . . . 55

3.4 .2 Structure solution of crystal form $\mathrm{S}$. . . . . . . . 57

3.4.3 Refinement of form $\mathrm{S}$ and structure solution of form $\mathrm{L}$. . 62

3.4 .4 Model quality . . . . . . . . . . . . . . . . 62

3.4 .5 Sulfamidase monomer . . . . . . . . . . . . . . . 63

3.4 .6 The homodimer . . . . . . . . . . . . . . . . 69

$3.4 .7 \quad$ Active site $\ldots \ldots \ldots \ldots$. . . . . . . . . . . . . . 69

3.4 .8 Comparison with O-sulfatases . . . . . . . . . . . 77

3.4.9 Comparison of the final model with the rotation-translation solution ................... 83

3.5 Discussion $\ldots \ldots \ldots \ldots \ldots \ldots$

3.5 .1 Sulfamidase monomer . . . . . . . . . . . . . . . . 83

3.5 .2 Sulfamidase dimer . . . . . . . . . . . . 84 
3.5 .3 Active site . . . . . . . . . . . . . . . 84

3.5 .4 Enzymatic reaction mechanism . . . . . . . . . . . . 86

3.5 .5 Glycosylation sites . . . . . . . . . . . . . . . . . 88

3.5 .6 Disease-causing mutations . . . . . . . . . . . . . . . 89

3.5.7 Molecular basis of mutations with a geographical distribution bias ................... 94

3.5 .8 Mutations affecting the dimer interface . . . . . . . . . . 97

3.5 .9 Limitations and prospects . . . . . . . . . . . . . . . . . 98

3.6 Conclusions $\ldots \ldots \ldots \ldots . \ldots \ldots 9 . \ldots \ldots$

\begin{tabular}{ll}
\hline Appendix & 103
\end{tabular}

\begin{tabular}{ll}
\hline Literature & 105
\end{tabular} 



\section{List of Abbreviations}

$\mu \quad$ micro

$\AA$

angstrom, $10^{-10} \mathrm{~m}$

$\mathrm{a}, \mathrm{b}, \mathrm{c}, \beta$

unit cell parameters for a monoclinic cell

ADP

adenosine diphosphate

Ala

alanine

$\operatorname{Arg}$

arginine

ASA

ASB

arylfulfatase A, also known as human lysosomal cerebroside-3-sulfate 3-sulfohydrolase

arylsulfatase B, also known as human lysosomal $\mathrm{N}$-acetylgalactosamine-4-sulfate 4-sulfohydrolase

Asn

asparagine

Asp

aspartate

asu

asymmetric unit

AvIDH

Azotobacter vinelandii isocitrate dehydrogenase

AvIDH-Holo

AvIDH holoenzyme

AvIDH-Iso

AvIDH in complex with isocitrate

$\mathrm{BcPMH}$

Burkholderia caryophilli PG2952 phosphonate monoester hydrolase

$\mathrm{CCP} 4$

Collaborative Computational Project, Number 4

$\mathrm{CgIDH}$

Corynebacterium glutamicum isocitrate dehydrogenase

CgIDH-Apo1

$\mathrm{CgIDH}$ apoenzyme 1

CgIDH-Apo2

CgIDH apoenzyme 2

CgIDH-Holo

CgIDH holoenzyme

Cys

cysteine 
EcIDH

ES

FGly

GAG

GALNS

Gln

Glu

Gly

$\mathrm{H}$ bond(ing)

His

IDH

Ile

$\mathrm{kDa}$

$\mathrm{L}$

Leu

Lys

M

Met

ML

MPS

MR

$\mathrm{NAD}^{+}$

$\mathrm{NADH}$

$\mathrm{NADP}^{+}$
Escherichia coli isocitrate dehydrogenase

human estrone/dehydroepiandrosterone sulfatase

formylglycine ( $\mathrm{L}-\mathrm{C}_{\alpha}$-formylglycine)

glycosaminoglycan; also known as mucopolysaccharide

human lysosomal ( $\mathrm{N}$-acetyl)galactosamine-6-sulfatase

glutamine

glutamate

glycine

hydrogen bond(ing)

histidine

isocitrate dehydrogenase

isoleucine

kilodalton

large cell (crystal form of SGSH)

leucine

lysine

molar

methionine

maximum likelihood

mucopolysaccharidosis

molecular replacement

nicotinamide adenine dinucleotide

nicotinamide adenine dinucleotide (reduced form)

nicotinamide adenine dinucleotide phosphate 
$\mathrm{NADPH}$

NASA

NCS

PAS

PDB

PEG

Phe

Pro

R-T

ReIDH

RMSD

S

SAXS

sc

SDS-PAGE

Ser

SGSH

$\mathrm{T}$

Thr

Tris

$\operatorname{Trp}$

Tyr nicotinamide adenine dinucleotide phosphate (reduced form)

normalized accessible surface area

non-crystallographic symmetry

Pseudomonas aeruginosa sulfatase

Protein Data Bank

polyethylene glycol

phenylalanine

proline

rotation-translation

isocitrate dehydrogenase from Ralstonia eutropha

root mean square deviation

small cell (crystal form of SGSH)

small-angle X-ray scattering

side chain

sodium dodecylsulfate polyacrylamide gel electrophoresis

serine

$\mathrm{N}$-sulfoglucosamine sulfohydrolase, also known as sulfamidase, sulfamate sulfohydrolase and heparan $\mathrm{N}$-sulfatase

$3_{10}$ helix

threonine

tris(hydroxymethyl)aminomethane

tryptophan

tyrosine 
valine

$\mathrm{w} / \mathrm{v}$

weight per volume 


\section{List of Figures}

$1.1 \quad$ X-ray interference pattern $\ldots \ldots \ldots \ldots$. . . . . . . . . . 4

1.2 An atomic model built into an electron density map . . . . . . . . 5

2.1 Reaction catalyzed by isocitrate dehydrogenase . . . . . . . . . 18

2.2 Coenzymes NADP ${ }^{+}$and NAD ${ }^{+} \ldots \ldots \ldots \ldots$

2.3 Crystal optimization trials . . . . . . . . . . . . . . . . . . . 21

2.4 Asymmetric unit with holo- and apoenzyme models of IDH. . . . 25

2.5 Domains and secondary structure elements in CgIDH-Holo . . . . 28

2.6 Comparison of CgIDH-Holo and -Apo1 and -Apo2 forms . . . . . 29

$2.7 \quad$ CgIDH-Holo and AvIDH-Holo . . . . . . . . . . . . . . . 30

2.8 Comparison of CgIDH-Holo and AvIDH-Holo $\ldots \ldots . . . . .30$

2.9 Hypothetical model of isocitrate in binding site at an interdomain

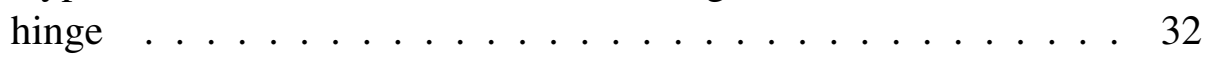

2.10 Hinge motion close to the isocitrate binding site. . . . . . . . . 32

$2.11 \mathrm{NADP}^{+}$omit map . . . . . . . . . . . . . . . 33

2.12 Stereo view of $\mathrm{NADP}^{+}$-binding interactions $\ldots \ldots \ldots$

2.13 Disruption of coenzyme binding in the apoenzyme in the asymmetric unit ...................... 44

3.1 Schematic structure of heparan sulfate . . . . . . . . . . 50

3.2 Sulfamidase purity $\ldots \ldots \ldots \ldots \ldots$. . . . . . . . . 52

3.3 High-resolution diffraction from a sulfamidase crystal . . . . . . . 54

3.4 Sulfamidase crystallization trials $\ldots \ldots \ldots \ldots$

3.5 Model bias at the rotation-translation solution stage . . . . . . . . 59

$3.6 \quad$ Model bias in another region of the map . . . . . . . . . . . . 60

3.7 Density pointing in biased, correct and noisy directions . . . . . . 61

3.8 Sequence mapped to secondary structure, SGSH . . . . . . . . . . 65

3.9 Topology diagram of SGSH $\ldots \ldots \ldots 7$

3.10 Secondary structure elements in a subunit . . . . . . . . . . 68

3.11 SGSH dimer, front view $\ldots \ldots \ldots$. . . . . . . . . . . . . . . . . . . . . . . . . .

3.12 SGSH dimer, top view . . . . . . . . . . . . . . . . 71

3.13 SGSH dimer, bottom view . . . . . . . . . . . . . 71

3.14 SGSH dimer, side view . . . . . . . . . . . . . . 72

3.15 SGSH dimers in crystal form L . . . . . . . . . . . . . . 73

3.16 SGSH dimer in front surface view with active site mapped onto the surface. . . . . . . . . . . . . . 74

3.17 Active site pocket in surface cleft. . . . . . . . . . . 75

3.18 Active site pocket. . . . . . . . . . . . . . 75

3.19 Stereo view of the active site. . . . . . . . . . . . . . . 76

3.20 Amino acid sequence comparison of the human sulfamidase . . . 79

3.21 Overlay of sulfamidase with sulfatases. $\ldots \ldots \ldots$. . . . . 80 
3.22 Comparison of SGSH with PAS . . . . . . . . . . . . 81

3.23 Comparison of SGSH with PAS, active site region. . . . . . . . . 82

3.24 Sulfamidase and the rotation-translation solution . . . . . . . . 83

3.25 Proposed reaction mechanism of SGSH. . . . . . . . . . . . . 87

3.26 Disease-causing missense mutations mapped onto SGSH structure. 95

3.27 Structural environment of Arg74, the site of a common mutation in MPS IIIA $\ldots \ldots \ldots \ldots 6$

3.28 Structural environment of Arg245, the site of a common mutation in MPS IIIA . . . . . . . . . . . . . . . . . . 97 


\section{List of Tables}

2.1 Data collection and refinement statistics, IDH . . . . . . . . 27

2.2 Comparison of CgIDH-Holo with other monomeric IDHs. . . . . 31

3.1 Data collection and refinement statistics, SGSH . . . . . . . . . 64

3.2 Structurally equivalent active site residues in SGSH and O-sulfatases 78

3.3 Missense mutations in MPS IIIA $\ldots \ldots \ldots$. . . . . . . . . . . . 90 



\section{Overview}

In an X-ray crystallographic experiment, intensities and directions of X-rays scattered by electrons in a crystal are measured. However, the relative phase information in the interference pattern is lost. If we knew the phases, we could calculate the electron density in the crystal, fit an atomic model to the density and thus solve the three-dimensional structure of the substance forming the crystal. One method of deriving the phase information is called molecular replacement (MR). In this method, the diffraction data are used to correctly position in the crystal a search model that is derived from a known atomic structure believed to be similar to the unknown target structure. Phases calculated for the correctly positioned model can then be combined with X-ray intensity data for the unknown structure to calculate a more or less hybrid electron density map. A crucial test is whether we are able to identify features corresponding to the target structure in this map as the phases come from a different structure. For proteins, if the sequence identity between the search and target structure is below $30 \%$, the method is often difficult or impossible to use and has typically failed. However, recent research is expanding this threshold and it continues to be an area of active research. Here, the method was used to attempt to solve crystal structures of two protein enzymes. In the first, the shared sequence identity between the search and target structures was $100 \%$, making the method straightforward to apply. In the second, the sequence identity was approximately $22 \%$. The method was successfully applied also in this case.

The first enzyme was the Krebs cycle enzyme isocitrate dehydrogenase from the bacterium Corynebacterium glutamicum. It is of special interest as it has been shown to recognize with high specificity its coenzyme nicotinamide adenine dinucleotide phosphate from the closely similar coenzyme nicotinamide adenine dinucleotide, which lacks a phosphate group that the former has, and has a hydroxyl group instead. Crystals of the enzyme were grown in the presence of the coenzyme. The crystal structure was solved to attempt to better understand how the high-specificity recognition is achieved at the atomic level.

Heparin and heparan sulfate are glycosaminoglycans (or mucopolysaccharides)-polysaccharides made up of a repeating disaccharide structural unit with many covalently bound sulfate groups. Their degradation in the body occurs in the cell organelles lysosomes. The deficient activity of one of the enzymes involved causes a progressive neurodegenerative disease known as mucopolysaccharidosis IIIA. It typically manifests itself in childhood, often leading to premature death in the second or third decade of life. Many mutations associated with the disease have been described. However, our understanding of how these mutations affect enzyme structure and function has been limited, one of 
the major hurdles being that the three-dimensional structure of the enzyme was unknown. Here, crystals of the enzyme were grown. The first structure of a sulfamidase, specifically the human enzyme, was solved using an MR search model that shared a low sequence identity with the target enzyme. The structural effects of the mutations are described, and an enzyme mechanism proposed. 


\section{Structure determination by X-ray crystallography: A background}

\subsection{Summary}

Ripples on the still surface of a pond help us figure out where perhaps a stone was dropped. Somewhat similarly, if we shoot X-rays through a crystal, the electrons in the crystal scatter X-rays. By measuring the direction and intensity attributes of the resulting interference pattern (Fig. 1.1), we can often infer the electron density at different points in the crystal. A 3-dimensional, often even atomic, model may then be fitted into the electron density, yielding a structure model of the substance forming the crystal (Fig. 1.2). As many different types of substances occur as crystals, including the sand under our feet, diamonds and gemstones, organic matter such as proteins and nucleic acids inside our bodies and even whole viruses, they are all in principle amenable to 3-dimensional or atomic structure determination using this method.

However, before we are able to do so, we have a problem to solve: In measuring only the intensities and directions of the interference pattern, we lose important information about the relative phase differences between the "reflections" (spots) in the interference pattern. The problem stems from the fact that, although detectors to measure X-ray intensities exist, those to measure the needed relative phases do not (Sherwood and Cooper, 2011). In an optical microscope, the optical lense recombines scattered light rays in appropriate phase relationships to produce an image that we can observe. Unfortunately, appropriate lenses for Xrays are not available either. Thus, we face what is known as the "crystallographic phase problem".

One method of solving the phase problem is to first identify (part of) a molecule (called a search model fragment) with a known structure that is similar to (a part of) the target structure that we wish to determine. Second, an attempt is then made, using the measured intensities, to place the search model fragment in the crystal in a position closely similar to the position occupied by the target molecule. Phases can now be calculated based on the atomic positions of the search model. Search model phases can then be combined with information derived from the measured intensities of the target structure to calculate a hybrid 3-dimensional electon density map. However, since the phases, which are crucial, were from a different structure, success depends on how well we are able to identify features of the target structure in this hybrid map. If we succeed, we can (slowly) build a structure model corresponding to the target molecule. This method of solving structures is known as molecular replacement. If the target molecule shares only a relatively distant similarity with the search model, the structure can be difficult 


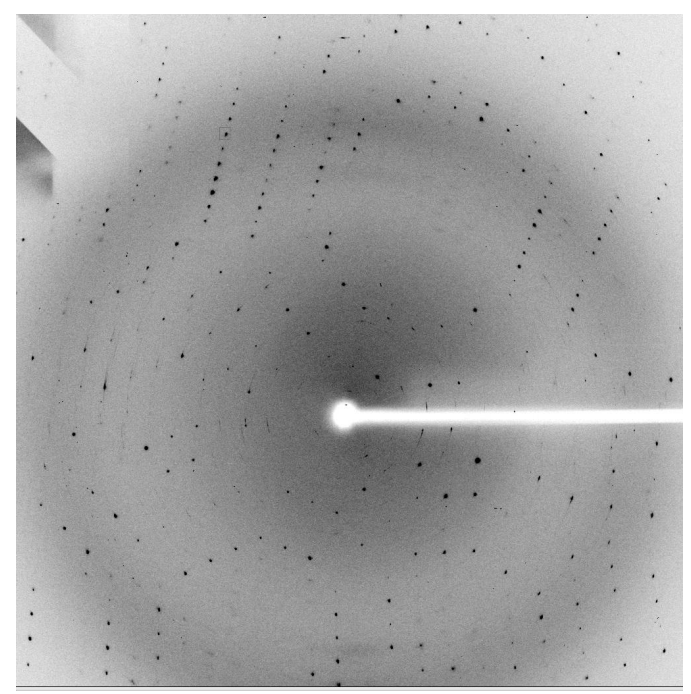

Figure 1.1: An X-ray interference pattern. X-rays diffracted by a protein crystal form an interference pattern with intensity peaks called reflections (black spots). The directions and intensities of reflections are measured. Crucial information on relative phases of reflections is lost.

or impossible to solve using this method.

\subsubsection{Introduction: The phase problem}

In contrast with ripples on the 2-dimensional surface of a pond, reflections in the $\mathrm{X}$-ray diffraction pattern can be thought of as populating a 3-dimensional space (reciprocal space). Each reflection in the interference pattern can be uniquely specified by three numbers known as its $h k l$ indices.

In the interference pattern, constructive interference occurs only in certain directions, resulting in reflections; interference being destructive in other directions. This can be understood as a consequence of Bragg's law, which states that

$$
\lambda=2 d \sin \theta
$$

where $\lambda$ is the wavelength of the radiation, $\theta$ is half of the angle by which the direction of the incoming radiation is changed, and $d$ is the distance between a set of parallel reflecting hkl planes in the crystal for a reflection with indices hkl. In other words, constructive interference only occurs for directions in which the right hand side of the equation takes values equal to integral multiples of the wavelength of the radiation. This is so because, in this case, the extra path travelled by the radiation in scattering from different planes in the given set of planes is an integral multiple of the wavelength. 


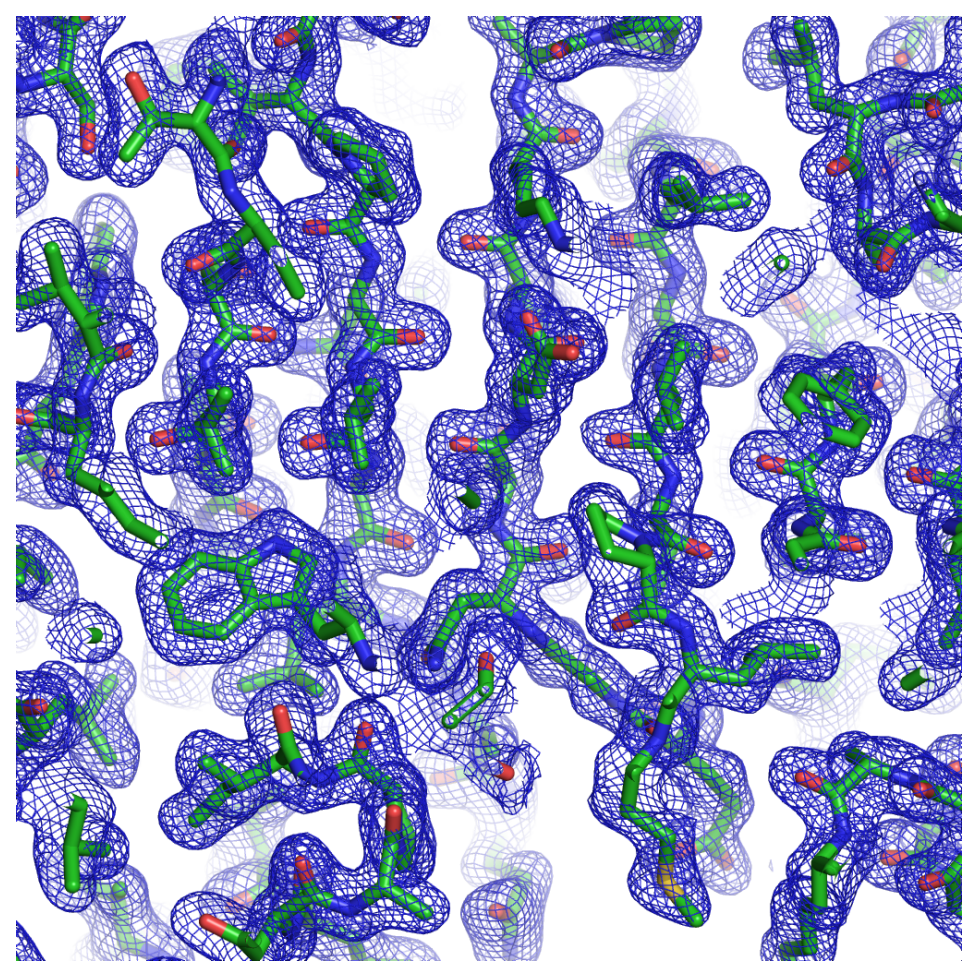

Figure 1.2: An atomic model built into an electron density map. The electron density map is shown as a blue mesh into which a 3-dimensional structure of a protein (green, red and blue sticks) was built. 
Theoretically, the electron density $\rho$ at a given point in the crystal with fractional coordinates $x y z$ can be calculated using the reverse Fourier transform equation as

$$
\rho(x y z)=\frac{1}{V} \sum_{h k l} F(h k l) \exp [-2 \pi i(h x+k y+l z)],
$$

where $\mathrm{V}$ is the volume of the unit cell of the crystal, and $F(h k l)$ is a property known as the structure factor of a reflection $h \mathrm{kl}$, which has two components, an amplitude $|F|$ and a phase $\phi$, both of which are explicitly expressed in the following form of equation (1.2),

$$
\rho(x y z)=\frac{1}{V} \sum_{h k l}|F(h k l)| \exp [i \phi(h k l)] \exp [-2 \pi i(h x+k y+l z)] .
$$

The structure factor amplitude is proportional to and derivable from the experimentally measured intensities $I$,

$$
|F(h k l)| \propto \sqrt{I} .
$$

However, the phases need to be determined before equation (1.3) can be used.

Fortunately, if we know the atomic structure of a substance, both its structure factor amplitudes and phases can be calculated using the forward Fourier transform equation (Clegg, 2006a). The structure factor $F$ of a given reflection $h k l$ is then given by

$$
F(h k l)=\sum_{j=1}^{N} f_{j} \exp \left[2 \pi i\left(h x_{j}+k y_{j}+l z_{j}\right)\right],
$$

where the sum is over all $\mathrm{N}$ atoms in the unit cell of the crystal, $f_{j}$ is the atomic scattering factor for the $j$ th atom with co-ordinates $x_{j}, y_{j}, z_{j}$. Thus, if we derived the phases from a structure that we believe is similar to another one that we wish to determine, we can use phases calculated from the known structure and try to interpret the resulting electron density in a reasonable manner to derive the target structure. This idea lies behind the molecular replacement method (below). However, in order to be able to do that, we first need to orient and place the known structure within the unit cell of the crystal of the unknown target structure. This is done using the Patterson method or using maximum-likelihood based methods.

\subsubsection{The Patterson method}

Although the first crystal structures were solved about a hundred years ago (see Bragg, 1914 for an overview), in the absence of a general theoretical solution to the phase problem, experimentally measured intensities were of rather limited use. Structures could only be determined for some of the simplest compounds 
(see Hauptman, 1991 for a review). As David Harker noted (Harker, 1936):

"The determination of the inner structure of a crystal by means of xrays has been, in most cases, a process of trial and error. Once the space group of the crystal is known, the direct use of the x-ray data is abandoned. A structure which satisfies the symmetry requirements of the space group is then assumed and the intensities of the diffracted $\mathrm{x}$ ray beams are calculated on this basis. If these do not agree with the experimentally determined intensities, another structure is assumed and the process is repeated. Systematic methods of carrying out this process have been devised which are remarkable for the rapidity and accuracy with which, in some cases, they lead to the elimination of every structure but the true one. In other cases, however, the systematic trial and error method breaks down and either fails completely or leads to the loss of much time."

Harker was writing here to contrast the method used to solve the first structures with the implications of a major discovery by AL Patterson, who showed (Patterson, 1934, 1935) that peaks of the function

$$
P(x y z)=\frac{1}{V} \sum_{h k l}|F(h k l)|^{2} \exp [2 \pi i(h x+k y+l z)]
$$

could be interpreted in 3 dimensions as those corresponding to interatomic vectors. Significantly, the peak height was directly proportional to the product of the atomic numbers of the atoms connected by the vector. Thus, if one or a few heavy atoms were present, peaks corresponding to these atoms dominated the map, and their coordinates could be determined directly or by some trial and error. At a time when Fourier series evaluation "to show the true form of the function $P(x, y, z)$ [had] never been carried out, the calculation involving a prohibitive amount of labor," Harker made the use of the Patterson method much more practical by showing that the Fourier series should be calculated for special planes (Harker planes) or lines that could be deduced from the space group. The solved structures were then validated by assessing the agreement between intensities calculated from the structure (above) and those observed experimentally.

Although this was a major development, it could only be used to determine structures of a small subset of compounds of interest. A major limitation of the method was that if no heavy atoms were present in a structure, attempts had to be made to incorporate one, with attendant issues. Another limitation had to do with the fact that for an electron density map with $n$ atomic peaks, the Patterson map has on the order of $n^{2}$ peaks. If more than a few heavy atoms were present, peak overlaps made atomic assignments difficult or impossible! 


\subsubsection{Direct methods}

It was only beginning in the 1950s that a general method to solve the phase problem was worked out (see Hauptman, 1991 for a review), especially including the tangent formula of Karle and Hauptman (1956). Briefly, if unknown phases could adopt arbitrary values, they would result in an arbitrary number of structures. However, the electron density in the crystal has non-negative values, which places constraints on values the phases can reasonably have. Additionally, for atomic resolution data, the density could be reasonably argued to consist of sharp peaks (atomic positions) separated by relatively flat regions (interatomic spaces), which is a powerful "atomicity" constraint. The number and types of atoms in a compound are often known and often the structure could be assumed to consist of more or less identical atoms (ignoring hydrogens to a first approximation as they only have a single electron each). These and other constraints translate into probabilistic relationships between measured intensities and phases on the one hand, and between phases of different reflections on the other. In combination with some trial and error, approximate phases for enough strong reflections are determined using these "direct methods" to produce an initial approximate electron density map. The density is then modified in real space (peaklist optimization) and phases refined in the reciprocal space using the tangent formula (dual space recycling) in many cycles to solve the structure. Currently, the structure is typically solved in the space group P1, which appears to help prevent phases from getting trapped in local minima (Sheldrick and Gould, 1995). The space group is determined subsequently (Burla et al., 2000; Palatinus and van der Lee, 2008).

The limitations of this otherwise powerful method are that it typically works for structures of up to approximately 1000 atoms, and data to approximately $1.2 \AA$ are in general needed (Sheldrick, 1990) due to the assumption of resolved atoms.

\subsection{Macromolecular structure determination}

Macromolecules often have orders of magnitude more atoms than small molecules and a much higher solvent content. Their crystals often diffract relatively poorly as compared with small molecules. However, the Patterson and direct methods and, more recently, maximum-likelihood based methods can be used to solve the phase problem for many macromolecules.

\subsubsection{Experimental phasing}

If two crystals differ mainly in the presence or absence of one or more marker atoms (e.g., heavy metals, halides, selenium as part of selenomethionine, or sulfur), the differences in structural factor amplitudes of reflections in the two crystals 
can be used as coefficients in the Patterson function to find the positions of these marker atoms in the Patterson map:

$$
P(u v w)=\frac{1}{V} \sum_{h k l}\left(F_{P M}-F_{P}\right)^{2}(h k l) \exp [2 \pi i(h x+k y+l z)]
$$

where $F_{P M}$ and $F_{P}$ are the structure factor amplitudes of the marker atom derivative and the native crystals, respectively. The phases of reflections derived from the marker atom positions in general constrain phases of the native dataset (without marker atom incorporated) reflections to one of two possibilities. The native phases can then be uniquely determined e.g. by making one or more marker atom derivatives, or using direct methods or density modification (Rupp, 2010, pp. 475-479), thus solving the macromolecular structure. The method may involve a single marker atom derivative (single isomorphous replacement, SIR) or multiple derivatives (multiple isomorphous replacement, MIR). Isomorphous replacement was used to solve the first macromolecular crystal structures.

Marker atoms also often cause small differences in the intensities of centrosymmetrically related reflections called Bijvoet pairs for a crystal measured at a single wavelength (single-wavelength anomalous dispersion, SAD). If these differences can be accurately measured, they indicate the positions of marker atoms in the Patterson function

$$
P(u v w)=\frac{1}{V} \sum_{h k l}\left(F_{P M}(h k l)-F_{P M}(-h-k-l)\right)^{2} \exp [2 \pi i(h x+k y+l z)],
$$

where $F_{P M}(h k l)$ and $F_{P M}(-h-k-l)$ are the structure factor amplitudes of the Bijvoet pairs. In addition, so-called dispersive differences in intensities for reflections can be obtained by collecting data at different wavelengths (multi-wavelength anomalous dispersion, MAD). Anomalous and dispersive differences can be used as such or in combination with isomorphous replacement (e.g. SIRAS, which is SIR combined with SAD) to uniquely determine phases of marker atoms and the native dataset. Finally, radiation-induced changes can also be used to derive phase information (RIP).

\subsection{Molecular replacement}

In molecular replacement (Rossmann and Blow, 1962), starting phases are derived from a molecule that is believed to be similar to the molecule whose structure we wish to solve. Assuming this assumption is valid, the search fragment needs to be accurately positioned first before the calculated phases can be used. This can be accomplished by using Patterson or maximum-likelihood (ML) based methods (for a review, see Evans and McCoy, 2008). The Patterson method will be 
described below, partly to continue its discussion and partly because (at least for the purposes of MR structure solution in crystallography) it lends itself to readier visualization as compared to the ML method thanks to the properties of the Patterson space. Following that, the methodological basis of the maximum-likelihood based method will be mentioned briefly.

A constraint that can at times be useful to solve structures is that peaks in a Patterson map derived from experimentally measured intensities of a target structure match those derived from all or part of a previously known atomic structure (search model fragment). This can be useful if we expect the two structures to share a significant degree of structural features. If successful, the constraint allows us to position the search model in the crystal such that its orientation and position are similar to those of the target molecule in the unknown structure and the derived phases can be used to calculate a map as indicated above. If our assumptions were correct, we may be able to identify features corresponding to the target structure in the resulting electron density, remodel the search model accordingly to calculate better phases and better maps in the next cycle, teasing out the target structure in an iterative procedure.

An important starting question then is: How do we identify structural similarities between two molecules when we do not know the structure of one of them?

Proteins are macromolecules that are composed typically of 20 types of amino acid units that covalently bind together to form linear chains (primary structure). The linear chains fold to form higher-order structures, including secondary, tertiary and quaternary structure. The amino acid sequence of two proteins can be compared to get an estimate of structural similarity. However, the sequence between related molecules can diverge significantly over the evolutionary time scale so that structural relationships can be hard or impossible to determine.

Chothia and Lesk (1986) found that the successful prediction of the structure of a protein on the basis of its sequence and the structure of a homologue can often be expressed as a function of the sequence identity. The root mean square deviation of main chain atoms, $\triangle$, is given by

$$
\triangle / \AA=0.40 \exp [1.87 H],
$$

where $H$ is the fraction of mutated residues. At approximately $20 \%$ homology, the structural changes could be large and impossible to predict. Additionally, $\beta$ sheet proteins were more variable than $\alpha$-helix proteins, likely as the former have a higher fraction of loops, which are more likely to undergo local refolding upon amino acid mutation.

In general, an appropriate search model needs to be both correctly oriented (3 degrees of rotational freedom) and located (3 degrees of translational freedom) in the unit cell. However, the properties of the Patterson space allow a simplification 
of the search for a solution in this 6-dimensional solution space. The search can be split into two sets of computationally easier 3-dimensional searches (Rupp, 2010), with three main steps (Rossmann, 1990): First, the molecule is rotated to correctly orient it (Rossman and Blow, 1962). Second, it is translated to the correct position in the unit cell. Third, the successfully oriented and translated molecule is used to determine starting, approximate phases as described above. When starting from a poor search model, a critical fourth step is whether it is feasible to bootstrap the target structure from a hybrid electron density map derived from target amplitudes but search model phases. The phases thus need to be improved.

\subsubsection{Rotation search}

In the Patterson map, peaks close to the origin correspond to intra-molecule interatomic vectors.

Intramolecular vectors in the Patterson space can be used to properly orient the search model. The molecule is rotated in small angular increments until an optimal agreement occurs between intramolecular vectors in a given orientation in the Patterson space and corresponding vectors calculated from the observed intensities. The optimal agreement can be measured using an overlap function (Rossmann, 2001) such as

$$
R\left(\theta_{1} \theta_{2} \theta_{3}\right)=\int_{U} P_{1}(\mathbf{x}) P_{2}\left(\mathbf{x}^{\prime}\right) d \mathbf{x}
$$

where $R\left(\theta_{1} \theta_{2} \theta_{3}\right)$ is the rotation function indicating a match between the two Patterson functions $\mathrm{P}_{1}(\mathbf{x})$ and $\mathrm{P}_{2}\left(\mathbf{x}^{\prime}\right)$ at rotation angles $\theta_{1}, \theta_{2}, \theta_{3}$ from an arbitrary starting orientation; the integration is over volume $\mathrm{U}$.

\subsubsection{Translation search}

In general, intermolecular vectors in Patterson space can serve to translate the oriented search molecule to the correct position with respect to the origin and various symmetry elements in the unit cell of a given space group. An exception is the space group P1, in which any point in the structure can serve as the origin (see e.g. Clegg, 2006b). Other space groups, e.g. P2 ${ }_{1}$, have a polar axis. The origin can float along this axis as there are no symmetry elements perpendicular to it that place constraints along this axis. The search model only needs to be correctly placed in the plane perpendicular to it; no search needs to be performed along one or more polar axes unless a part of the structure is being added to a previously placed part, in which case the new part needs to be placed in the correct relation to the previous one. Algorithms to perform translation search vary. Conceptually, 
the correctly oriented search fragment can be seen to be placed at different points in the cell and the calculated and observed Patterson maps compared.

Once a rotation-translation solution is found, its calculated phases are used in combination with target structure factor amplitudes to calculate an initial electron density map.

\subsubsection{Bootstrapping the target structure}

If we start from a poor search model and we are unable to use its calculated phases to identify features of the target structure in the resulting map, the "rotationtranslation" solution has to be abandoned.

Sources of errors in the MR solution include poor data quality, correlation between rotation and translation, invalid constraints (assumption of identical search and target molecules) and the ratio between the sizes of the search model and the unit cell (Navaza, 1994). Indicators of a poor search model include a search model that is significantly incomplete, inaccurate or imprecise, contains many independent domains, and a low (less than 30\%) sequence identity with the target structure (Fokine et al., 2003).

Apart from rebuilding the model, the model can also be improved by refinement. As the measured quantities are diffraction intensities but the properties of interest are atomic parameters, these have to be derived from the measured properties. One method of deriving best parameters from observed data is through least-squares refinement (see Main, 2006 for an overview). Given data to a high resolution (e.g. for small molecules), the least-squares method works well. However, it assumes a Gaussian error distribution that may not be valid for macromolecular data, where data are often only available to lower resolution. Here, the more general maximum-likelihood method is used, which uses a probabilistic approach. The basic premise here is that the best model is the one that explains the data given with the greatest probability (McCoy, 2004). It has been implemented for both molecular replacement and refinement.

The method is based on Bayes' theorem, which states that the probability of a hypothesis, or of a model in estimating parameters, $P(H)$, given data $\mathrm{D}$ and conditioning information I is given (Sivia \& Skilling, 2006) by

$$
P(H \mid D, I)=\frac{P(D \mid H, I) \cdot P(H \mid I)}{P(D \mid I)} .
$$

In other words, the posterior probability

$$
P(\text { hypothesis } \mid \text { data }, I) \propto P(\text { data } \mid \text { hypothesis }, I) \cdot P(\text { hypothesis } \mid I)
$$

where the first term on the right hand side is the likelihood function and the second 
the prior probability of the hypothesis before the data are taken into account.

An unobserved variable can be used in intermediate calculations and then integrated out:

$$
P(A \mid I)=\int_{-\infty}^{+\infty} P(A, B \mid I) d B .
$$

This allows for optimal estimation of errors, e.g. by taking phases into account in calculating probabilities of structure factors derived from a model, but then integrating them out for optimal comparison with the observed data (intensities) as the phases were not measured (McCoy, 2004).

In practice, for computational convenience, logarithms of probabilities are used. The log-likelihood function can then be calculated as the sum of the logarithms of individual probabilities (assuming independent experimental observations) (Murshudov et al., 1997). Maximum-likelihood-based molecular replacement and refinement are implemented in the CCP4 (Collaborative Computational Project, Number 4, 1994) programs PHASER (McCoy et al., 2007) and REFMAC (Murshudov et al., 1997, 2011), respectively.

In one mode of molecular replacement implemented in the program ARCIMBOLDO (Rodríguez et al., 2009, 2012), multiple solutions are found for model $\alpha$-helices using PHASER. Density modification and chain tracing in SHELXE (Thorn and Sheldrick, 2013) is then used in an attempt to expand the structure. Other approaches that have been successfully applied in MR at low sequence identity include chain tracing in combination with modelling based on energy calculations using ROSETTA (DiMaio et al., 2011). Tertiary structure information from the available structures in the PDB has been used for MR in the implementation called BORGES (Sammito et al., 2013).

To judge whether we are making progress over the course of model rebuilding and refinement, the statistic crystallographic residual ( $\mathrm{R}$ value) can be calculated. It is defined as

$$
R=\frac{\sum_{\mathbf{h}}\left|F_{o}-k F_{c}\right|}{\sum_{\mathbf{h}} F_{o}} .
$$

However, this statistic can be arbitrarily lowered by overfitting, i.e., by fitting the model to noise. The risk of overfitting is higher if high resolution data are not available. In macromolecular crystallography, cross-validation is performed using 
the statistic $\mathrm{R}_{\text {free }}$ (Brünger, 1992), which is defined as

$$
R_{T}^{\text {free }}=\frac{\sum_{\mathbf{h} \in T}\left|F_{o}-k F_{c}\right|}{\sum_{\mathbf{h} \in T} F_{o}} .
$$

Here, the data are divided into two subsets, a working set that consists of approximately $95 \%$ of the data and a test set that has the rest. $\mathrm{R}_{\text {free }}$ is calculated for the test set of reflections, $\mathrm{T}$. Model parameters are refined only against the working set. Reflections corresponding to the test set can then be calculated from the model and used for validation against their observed values unused in refinement.

\subsection{Density modification}

The starting electron density map often has many errors. It may conflict with reasonable expectations for good macromolecular electron density maps. In this case, reasonable constraints can be applied to it. These constraints can help improve the phases and make the map better interpretable for the next cycle of structuremodel building. Constraints are often applied iteratively. This procedure is in general called density modification (see Zhang et al., 2012 for a review). Not all constraints may be applied in all cases, depending partly also on differences in implementations in different software.

Crystallographic symmetry operations are applicable to the entire crystal, including all unit cells and the symmetry elements contained in them. However, often that is not all the symmetry that can be recognized in a crystal. There are some symmetry operations that may be applicable to only part of each asymmetric unit in the unit cell. A molecule with a 5-fold rotation axis is an obvious example of non-crystallographic symmetry (NCS). However, NCS may also include rotation axes that can, at other times, be symmetry elements of a crystal space group, including e.g. 2-fold rotation axes.

NCS offers the possibility of increasing the signal to noise ratio in a density map. To this end, the NCS operators are determined first. One possibility for their use is to substitute electron density values at equivalent points in the NCS-related molecules by the averaged value at these points for all the molecules. The power of this constraint increases with increasing number of NCS-related molecules. In the case of some viruses, for instance, phases can be determined ab initio by applying NCS constraints. In an alternative procedure incorporated in the program SHELXE (Sheldrick, 2002, 2008, 2010), NCS-averaging can be combined with automatic chain tracing by applying NCS to model fragments instead of the density. Thus, better-traced NCS molecules help in tracing those in worse density. 
As part of density modification in SHELXE, splicing of two merging traced chains such that the better half at each end is retained at the expense of the worse half has also been seen to efficiently improve map quality. Density may be modified depending on the probability that a particular volume element contains an atom based on density variance at a surface $2.42 \AA$ from the element (sphere-ofinfluence algorithm implemented in SHELXE; Sheldrick, 2010).

Among other procedures in density modification (Zhang et al., 2012), electron density in the solvent region may be identified, a solvent mask generated and this density flattened (solvent flattening) to minimize noise. The distribution of density values in the non-solvent, macromolecular part can be adjusted to better match ideal probability distribution derived from a structure that has a similar solvent content and resolution (histogram matching). The density can be interpreted as atoms and their parameters refined (atomization). Protein backbone may be assumed to be connected (skeletonization). Especially when atomic resolution is available, constraints similar to those used in direct methods can be applied, as mentioned above.

Another use of density modification has to do with addressing what is often a significant issue in molecular replacement. As the initial phases in molecular replacement are derived from the search model fragment, the resulting map is biased towards the search model rather than the target model. This is known as model bias. One way it can be reduced is by excluding a region from structure factor calculations (Hodel et al., 1992 and references therein) or by using a probabilistic function to adjust the density based on expectations for a macromolecule (Terwilliger, 2004; also see Read, 1986).

Programs used for density modification in X-ray crystallography include DM (Cowtan, 1994), SHELXC/D/E (Sheldrick, 2010), and RESOLVE (Terwilliger, 2000). 



\section{Structural study of coenzyme specificity in an isoc- itrate dehydrogenase}

\subsection{Summary}

Protein enzymes catalyze a diverse array of reactions in biology using a limited set of amino acid residues, a challenging constraint. To overcome this, helper small molecules that facilitate enzymatic function have co-evolved with enzymes, including "coenzymes" such as nicotinamide adenine dinucleotide phosphate $\left(\mathrm{NADP}^{+}\right)$. The helper function implies the plausible existence in the enzyme of a mechanism for molecular recognition of the "correct" coenzyme and for its differentiation from closely similar incorrect ones.

$\mathrm{NADP}^{+}$differs from the closely similar coenzyme $\mathrm{NAD}^{+}$in having a phosphate group covalently bound at the $2^{\prime}$ position of the adenosine ribose; the latter has a hydroxyl group at this position. The former coenzyme is typically preferred by the bacterial isocitrate dehydrogenase (IDH) enzymes. Indeed, the enzyme from the bacterium Corynebacterium glutamicum has one of the highest described specificities for its coenzyme $\mathrm{NADP}^{+}$, preferring it to $\mathrm{NAD}^{+}$by a factor of 50 000 .

In the present study, crystals of the enzyme were grown in the presence of the coenzyme and the structure solved using molecular replacement. Part of the coenzyme was found well bound to the enzyme, including the crucial phosphate of $\mathrm{NADP}^{+}$. The enzyme residues mediating specific interaction with the coenzyme could therefore be identified.

A chance finding was that two enzyme molecules of this monomeric enzyme were obtained together in the same crystal, both differing in conformation by an interdomain rotation of $13^{\circ}$. The rotation could be pinpointed to two hinge points, one of which is located in tight proximity to the substrate (isocitrate) binding site of the enzyme. A model is therefore proposed in which binding of a small substrate molecule leads to large-scale conformational changes in this enzyme, leading to a closed, active conformation. It is also suggested that large hinge movements allow possible diversification in monomeric IDHs with respect to the mode of coenzyme binding: An unbound nicotinamide moiety does not appear to be a necessary condition for the binding of the substrate, as appears to be the case in the dimeric enzyme from Escherichia coli. Rather, the large hinge movement allows for the coenzyme to be well bound before the substrate. This model is consistent with the observed mode of coenzyme binding in the only previous holoenzyme (enzyme bound to coenzyme) structure of a monomeric IDH. It is speculated that concerted inhibition by glyoxylate and oxaloacetate could also involve a change in conformation of the enzyme to an inactive conformation. 

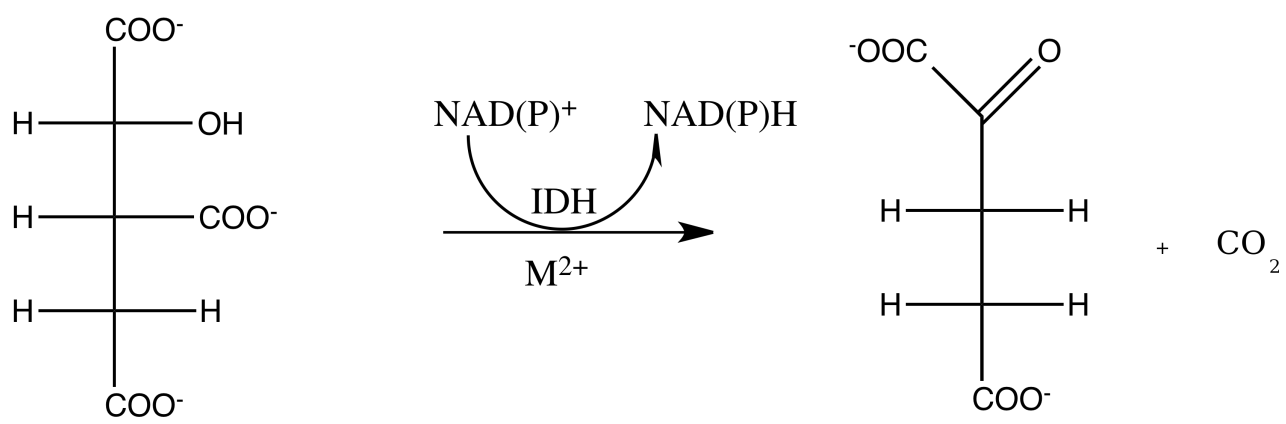

Figure 2.1: A schematic of the overall reaction catalyzed by IDH. Oxidative decarboxylation of isocitrate to produce 2-oxoglutarate and $\mathrm{CO}_{2}$. The coenzyme $\mathrm{NAD}(\mathrm{P})^{+}$is reduced to $\mathrm{NAD}(\mathrm{P}) \mathrm{H}$. A divalent metal ion $\left(\mathrm{M}^{2+}\right.$ acts as a cofactor.

\subsection{Introduction}

\subsubsection{Citric acid cycle and isocitrate dehydrogenase}

The citric acid cycle (Krebs and Johnson, 1937), also known as Krebs cycle or tricarboxylic acid cycle, is a key pathway of carbon metabolism in aerobic organisms. One molecule of a $\mathrm{C}_{2}$ (two-carbon) species, acetate, enters the cycle to be oxidized to two molecules of a $\mathrm{C}_{1}$ species, $\mathrm{CO}_{2}$. Other products of the cycle are utilized for metabolism, biosynthesis or energy production.

The first redox and $\mathrm{CO}_{2}$-generating steps involve the conversion of $2 R, 3 S$ isocitrate into 2-oxoglutarate, which is coupled to the reduction of the coenzyme nicotinamide adenine dinucleotide (phosphate) $\left(\mathrm{NAD}(\mathrm{P})^{+}\right)$to $\mathrm{NAD}(\mathrm{P}) \mathrm{H}$ (Fig. 2.1). Both steps are catalyzed by the enzyme isocitrate dehydrogenase (IDH) and utilize a divalent metal ion $\left(\mathrm{M}^{2+}\right)$, typically $\mathrm{Mg}^{2+}$ or $\mathrm{Mn}^{2+}$, as cofactor. Both atoms of carbon entering the citric acid cycle are lost to the environment as $\mathrm{CO}_{2}$, making the IDH step an important step of commitment. Indeed, it is a focus of regulation of the cycle (for a review for the enzyme used in the present study, see Gerstmeir et al., 2003). In order to be able to grow on acetate, or ethanol and fatty acids that are convertible to acetate, as the only sources of carbon, plants and bacteria can bypass the $\mathrm{CO}_{2}$-generating steps in a version of the cycle called the glyoxylate bypass, conserving both carbons (Kornberg and Krebs, 1957; see Kornberg, 1966 for a review). The first enzyme of the glyoxylate bypass, isocitrate lyase is important for the persistence of M. tuberculosis in mice (McKinney et al., 2000). For the bypass to run efficiently in the bacterial model E. coli, however, IDH needs to be inhibited. The Krebs cycle and the glyoxylate bypass may run in parallel to balance anabolic and catabolic requirements of E. coli (Dean and Golding, 1997). 


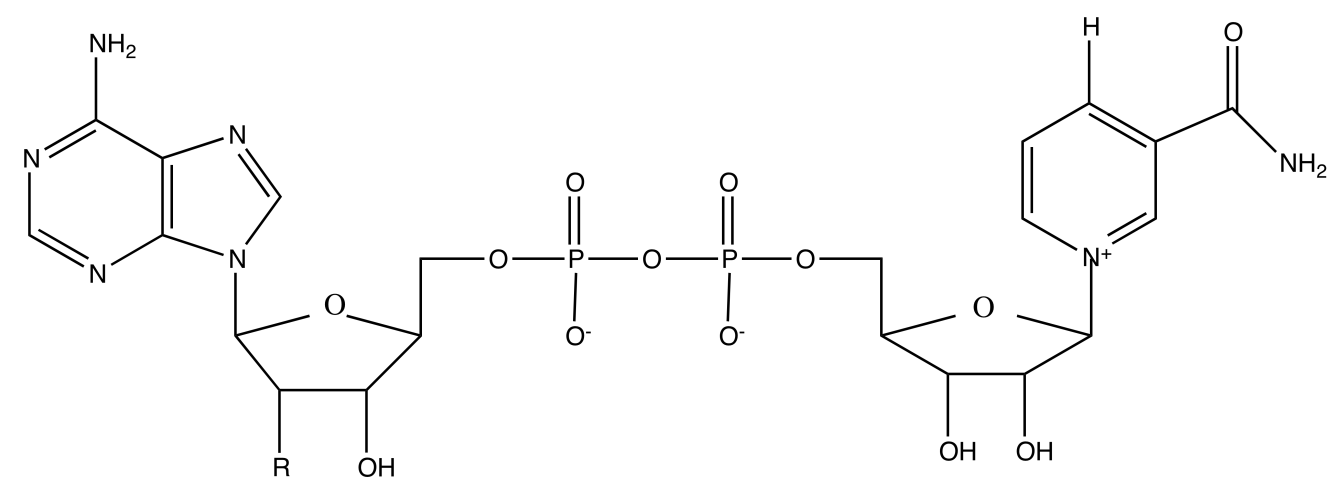

$\mathrm{NADP}^{+} \quad \mathrm{R}=\mathrm{O}-\stackrel{\mathrm{O}}{\mathrm{P}}_{\mathrm{O}}^{\mathrm{O}} \mathrm{O}$

$\mathrm{NAD}^{+} \quad \mathrm{R}=\mathrm{H}-\mathrm{O}$

Figure 2.2: A schematic of the structures of coenzymes $\mathrm{NADP}^{+}$and $\mathrm{NAD}^{+}$. $\mathrm{NADP}^{+}$has a phosphomonoester group (R) at the $2^{\prime}$ position of the adenosine ribose in contrast with the hydroxyl of $\mathrm{NAD}^{+}$.

\subsubsection{Coenzyme specificity}

Although IDHs as a set are able to use either $\mathrm{NADP}^{+}$or $\mathrm{NAD}^{+}$as the coenzyme in the redox step of the two-step reaction they catalyze, a given IDH tends to show a degree of preference for one or the other coenzyme, with bacterial IDHs typically preferring $\mathrm{NADP}^{+}\left(\mathrm{NADP}^{+}\right.$-IDH; EC 1.1.1.42) (Chen and Gadal, 1990). NADP ${ }^{+}$ and $\mathrm{NAD}^{+}$are similar; they differ in that the former has a phosphate group covalently attached to it at the $2^{\prime}$ position of the adenosine ribose while the latter has a hydroxyl group at this position (Fig. 2.2). Chen and Yang (2000) suggested that the phosphate group offers a greater possibility for specific interactions than a hydroxyl group, consistent with a greater coenzyme specificity observed in general for $\mathrm{NADP}^{+}$-dependent dehydrogenases as compared with $\mathrm{NAD}^{+}$-dependent ones. Among the former, $\mathrm{NADP}^{+}$-IDHs appeared to be especially specific to their coenzyme. Two of the most $\mathrm{NADP}^{+}$-specific IDHs that have been described are an $80 \mathrm{kDa}$ monomeric IDH product from the Streptomyces lividans (Zhang et al., 2009) and the monomeric IDH from Corynebacterium glutamicum (CgIDH) (Eikmanns et al., 1995; Chen and Yang, 2000). The former prefers $\mathrm{NADP}^{+}$to $\mathrm{NAD}^{+}$ by a factor of 60000 (with $\mathrm{Mg}^{2+}$ as cofactor) to 85000 (with $\mathrm{Mn}^{2+}$ ); the latter enzyme by 50000 (with $\mathrm{Mg}^{2+}$ ). 
Most bacterial IDHs are homodimeric (Chen and Gadal, 1990). Monomeric IDHs of approximately 80-100 kDa molecular weight have been described only in some bacteria, including A. vinelandii (Chung and Franzen, 1969), Colwellia maris (Ochiai et al., 1979; Ishii et al., 1987), R. vannielii (Leyland and Kelly, 1991), Vibrio parahaemolyticus (Fukunaga et al., 1992), C. glutamicum (Eikmanns et al., 1995) and Desulfobacter vibrioformis (Steen et al., 1998). CgIDH shares a relatively high sequence identity (of approximately 57-62\%) and similarity $(76-81 \%)$ with other monomeric IDHs. In contrast, monomeric IDHs share a low structure-based sequence homology of $7-8 \%$ with homodimeric IDHs (Yasutake et al., 2002). However, the tertiary structures of monomeric IDHs have been shown to be remarkably similar (Yasutake et al., 2002; Imabayashi et al., 2006) to that of the homodimeric EcIDH (Hurley et al., 1989).

\subsubsection{Structural studies of coenzyme binding in IDHs}

Structural studies of coenzyme binding have been described for many IDHs, including those for homodimeric ones such as NADP ${ }^{+}$-IDH from E. coli (EcIDH) (Hurley et al., 1991; Stoddard et al., 1993, 1998; Bolduc et al., 1995; Mesecar et al., 1997; Gonçalves et al., 2012), human cytosolic NADP ${ }^{+}$-IDH (Xu et al., 2004; Dang et al., 2009; Yang et al., 2010), NADP ${ }^{+}$-IDH from Aeropyrum pernix (Karlström et al., 2005), mitochondrial $\mathrm{NADP}^{+}$-IDH from Saccharomyces cerevesiae (Peng et al., 2008) and $\mathrm{NAD}^{+}$-IDH from Acidithiobacillus thiooxidans (Imada et al., 2008).

Two studies of coenzyme binding have been described in monomeric IDHs, for Azotobacter vinelandii $\mathrm{NADP}^{+}-\mathrm{IDH}$ (AvIDH) (Yasutake et al., 2003) and for the $\mathrm{NADP}^{+}$-IDH from Corynebacterium glutamicum (CgIDH) (Sidhu et al., 2011). Additionally, the crystal structure of AvIDH in complex with isocitrate (AvIDH-Iso) (Yasutake et al., 2002) and of CgIDH apoenzyme (CgIDH-Apo1) (Imabayashi et al., 2006) have also been described. Here, the structural mediators of the high $\mathrm{NADP}^{+}$specificity of CgIDH (Sidhu et al., 2011) are explored, and compared with some of the other IDHs.

\subsection{Materials and methods}

\subsubsection{Crystallization}

The gene for wild-type CgIDH, icd, was a gift from the laboratory of Prof. Bernhard Eikmanns, University of Ulm, Germany, where it was initially isolated and cloned (Eikmanns et al., 1995). Using this clone, the protein was overexpressed and purified as described (Audette et al., 1999; Bai et al., 1999; Imabayashi et al., 2006) by Fumie Imabayashi in the laboratory of Prof. Louis Delbaere. Pu- 


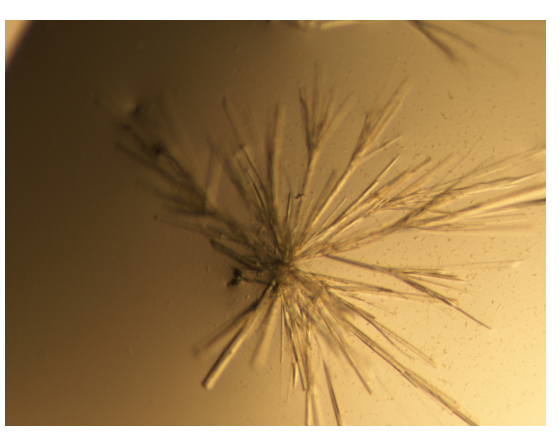

(a) Needles.

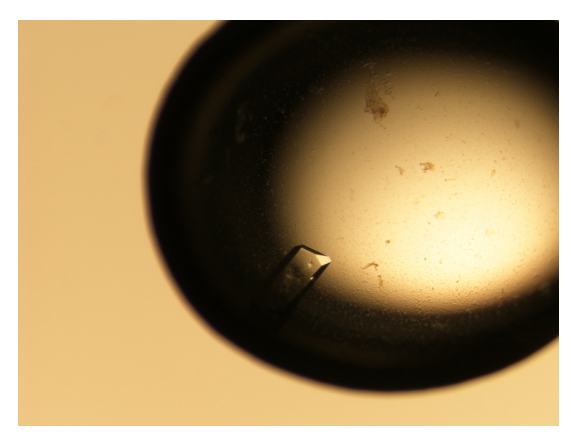

(b) Single crystal.

Figure 2.3: Crystal optimization trials. A fragment of the second crystal was used to solve the structure. The rod-shaped crystals grew to a maximum size of approximately $200 \mu \mathrm{m}$ x $50 \mu \mathrm{m}$ x $50 \mu \mathrm{m}$.

rified $\mathrm{CgIDH}$ was stored in small batches at $-80^{\circ} \mathrm{C}$ in a solution of concentration $10 \mathrm{mg} / \mathrm{mL}$ in $25 \mathrm{mM}$ 2-morpholineethanesulfonic acid buffer (pH 6.2) with $2.5 \mathrm{mM} \mathrm{MnSO} 4,2.5 \mathrm{mM}$ dithiothreitol and $10 \%$ glycerol.

For crystallization trials, solution from a batch was thawed, diluted with an equal volume of distilled autoclaved water (the $5 \mathrm{mg} / \mathrm{mL} \mathrm{CgIDH}$ solution) and used in entirety, without refreezing. The trials were conducted using the hangingdrop vapor diffusion method at a temperature of $295 \mathrm{~K}$. The reservoir (well) solution had a total volume of $0.5 \mathrm{~mL}$ and consisted of $25 \%(\mathrm{w} / \mathrm{v})$ polyethylene glycol 2000 monomethyl ether (PEG $2000 \mathrm{MME}$ ), 0.2 M tris(hydroxymethyl)amino methane $\cdot \mathrm{HCl}$ (Tris buffer) ( $\mathrm{pH} 7.3$ ), and $0.2 \mathrm{M} \mathrm{MgCl}_{2}$. To prevent ligand wastage, a second solution was made with the same composition as the reservoir solution but with $10 \mathrm{mM} \beta-\mathrm{NADP}^{+}$(Sigma-Aldrich) added. This second solution was mixed $1: 1$ with the $5 \mathrm{mg} / \mathrm{mL}$ CgIDH solution to form drops of a total volume of $2 \mu \mathrm{L}$. Crystals of different habits formed in three days to two months (Fig. 2.3a,b).

Crystals were difficult to cryoprotect, typically resulting in streaks in the Xray diffraction pattern, especially in the direction corresponding to the largest cell dimension in real space. In the successful trial (Fig. 2.3b), two solutions were used with the composition of the appropriate reservoir solution but with 20 and $30 \%$ glycerol added. To a drop with a CgIDH crystal, approximately $2 \mu \mathrm{L}$ of the reservoir solutions was added in two steps of increasing glycerol concentration. The crystal cracked into one large and two small fragments. Within 1 minute, the large fragment was harvested and flash-frozen in liquid $\mathrm{N}_{2}$. The gap between crystallization setup and freezing was 5 weeks for this crystal, which was successfully used for data collection. 


\subsubsection{Data collection}

Single crystal X-ray diffraction data were collected at the 08ID-1 beamline, Canadian Light Source, Saskatoon, University of Saskatchewan, using a wavelength of $0.9793 \AA$ on a MarMosaic $225 \mathrm{~mm}$ CCD detector. An oscillation range of $0.30^{\circ}$ per image was used to collect 633 images overall. The crystal diffracted to a resolution of $1.8 \AA$. However, due to a low completeness in the 1.8-1.9 $\AA$ resolution shell, data were processed only to $1.9 \AA$. Data reduction and scaling were performed using XDS (Kabsch, 2010) and XSCALE. The test set for cross validation was generated by randomly setting aside $5 \%$ of the data (5992 reflections). The crystal space group was determined to be $\mathrm{C} 2$, with 2 molecules in the asymmetric unit (data collection and processing statistics in Table 2.1).

\subsubsection{Structure solution and refinement}

The structure was solved using molecular replacement (MR) as implemented in the CCP4 (Collaborative Computational Project, Number 4, 1994) program PHASER (McCoy et al., 2007). The previously solved CgIDH apoenzyme structure (Imabayashi et al., 2006), Protein Data Bank (PDB) (Berman et al., 2000) accession code 2B0T (CgIDH-Apo1) was used as the search model. Using the coordinates of $\mathrm{CgIDH}-\mathrm{Apo} 1$ as a whole was unsuccessful. The coordinates were then divided into two rigid domains, one consisting of residues 2-138 and 558736 , and the second one of residues 139-557, leading to a solution with no major clashes.

Structure visualization and building was performed using the program COOT (Emsley et al., 2010); maximum-likelihood refinement of the coordinates and B factors against the working set data using the program REFMAC 5.5 (Murshudov et al., 1997), part of the CCP4 suite. The starting $\mathrm{R}_{\text {work }}$ and $\mathrm{R}_{\text {free }}$ were $32.0 \%$ and $31.5 \%$, respectively. In the beginning of the refinement, 2 -fold noncrystallographic symmetry (NCS) averaging with tight restraints was used. The restraints were then relaxed; no restraints were used in the final cycles of refinement. Protein $\phi$ and $\psi$ angles were not restrained during refinement. Indicators used to decide on optimal refinement and model building strategy included $\mathrm{R}_{\text {free }}$ (Brünger, 1992), free log likelihood, and results of model validation using the MOLPROBITY server (Davis et al., 2007; Chen et al., 2010).

Water molecules were added using the program COOT either automatically or manually. Waters were removed, as a rule, if the refined position of the nearest atom was either shorter than $2.3 \AA$ (except for $\mathrm{Mg}^{2+}$ and its ligands and for correlated half occupancies) or farther than $3.5 \AA$, and if there was poor electron density or the water $B$ factors refined to high values.

Towards the end, hydrogen atoms were added in riding positions and TLS 
anisotropic displacement parameters (Schomaker and Trueblood, 1968) refined. The TLSMD server (Painter and Merritt, 2006) was used to ascertain the approximately optimal number of TLS groups. Three TLS groups were defined for each monomer in the asymmetric unit: residues 2-138, 139-557 and 558-736. Upon the convergence of TLS parameters, atomic coordinates and individual B factors were refined (Winn et al., 2001). The TLSMD server was also used to validate the final TLS parameters, which were analyzed using the program TLSANL (Howlin et al., 1993), part of the CCP4 suite. TLS refinement and the addition of hydrogens led to significantly better values of $\mathrm{R}_{\text {free }}$.

$\mathrm{NADP}^{+}$coordinates were imported from the REFMAC monomer library as implemented in COOT. Its occupancy was estimated at 0.9 using trial and error, indicators used included the difference electron density and refined B factors of the ligand and neighboring protein atoms. A few partial occupancy side-chain rotamers were built into the model.

\subsubsection{Analysis of refined structure}

Secondary structure assignment was performed based on the DSSP algorithm (Kabsch and Sander, 1983) as implemented on the WHATIF server at http://swift.cmbi.ru.nl/whatif/. The latter was also used to determine crystal packing contacts. Hinge points between protein domains were determined using the program DYNDOM (Hayward and Berendsen, 1998), part of the CCP4 suite. Structure overlays and RMSD calculation was done using LSQKAB (Kabsch, 1976), part of CCP4. To calculate changes in inter-domain angles between monomers, their Domains I were superimposed followed by the calculation of the rotation required to superimpose Domains II. To evaluate hydrogen bonding, hydrogens were added using the MOLPROBITY server. Distances and angles were calculated using COOT; approximate angles between aromatic ring planes were calculated using an in-house program (NSS, unpublished). Figures were rendered using the program PYMOL (DeLano, 2002).

Sequences of (putative) monomeric IDHs from 21 species (referred to below as "representative sequences") were found using the National Center for Biotechnology Information (NCBI) server. Sequence alignments were done using the program INDONESIA (Madsen et al., 2002).

\subsection{Results}

\subsubsection{Structure solution}

The crystal structure of monomeric isocitrate dehydrogenase from Corynebacterium glutamicum ( $\mathrm{CgIDH})(80 \mathrm{kDa} ; 738$ amino acids) as co-crystallized with 
$\mathrm{NADP}^{+}$and $\mathrm{Mg}^{2+}$ was solved and refined against data to a resolution of $1.9 \AA$. The structure was solved using molecular replacement (MR) using the previously solved apoenzyme structure for the enzyme (CgIDH-Apo1; Imabayashi et al., 2006; PDB accession code 2B0T) as the search model. There are two enzyme molecules in the asymmetric unit (Fig. 2.4), one of them in the holoenzyme form, the other in as the apoenzyme. The coordinates and structure factor data were deposited in the PDB (accession code 3MBC).

Each enzyme chain has 735 amino acid residues (2-736 in the sequence). A partially modeled $\mathrm{NADP}^{+}$molecule is bound to chain A (the holoenzyme, CgIDH-Holo), none to chain B (the apoenzyme, CgIDH-Apo2). $\mathrm{A} \mathrm{Mg}^{2+}$ ion occupies the metal binding site in each enzyme molecule. A total of 614 oxygen atoms of water molecules were modeled into chain A, 182 in chain B, totaling 796 (number unadjusted for occupancy).

\subsubsection{Model quality}

Electron density for the holoenzyme chain was of significantly better quality (also with more waters modeled) than that for the apoenzyme. Part of the reason appears to be that, in the former, 50 residues form crystal packing contacts whereas only 30 do so in the latter. Significantly poor density was observed for the surface residue Asp306 in both chains but especially so in the apoenzyme; it was included in the model. Unincluded, however, were parts of some surface side-chains in the apoenzyme, which lay in poor density.

Electron density for a third of the coenzyme was clear and sharp; density corresponding to the middle third was of a partially disordered quality; no electron density was observed for the distal third. The first two thirds were judged for inclusion into the model but without disorder; the last third was not included in the model. The first third corresponds to the adenosine- $2^{\prime}$-phosphomonoester part of $\mathrm{NADP}^{+}$. Poorer density was observed in the neighborhood of the C5 atom of the ribose. The density corresponding to the $5^{\prime}$-diphosphate moiety was better again but less ordered than for the adenosine ribose part. No clear density corresponding to the nicotinamide nucleoside moiety of $\mathrm{NADP}^{+}$was observed. Attempts to model this moiety were unsuccessful.

To utilize them as indicators of model quality, protein $\phi / \psi$ angles were not restrained during the refinement. The Ramachandran plot (Ramakrishnan and Ramachandran, 1965; Lovell et al., 2003) displays 97.9\% of amino acid residues in the favored, $2.1 \%$ in the allowed, and none in the outlier region. The final $\mathrm{R}_{\text {free }}$ and $\mathrm{R}_{\text {work }}$ values were $22.9 \%$ and $18.9 \%$, respectively; the MOLPROBITY score 1.47 (96th percentile) (Davis et al., 2007; Chen et al., 2010). Table 2.1 gives the data processing, refinement and validation statistics. The structure of the CgIDHHolo (Holo) will be described below by default. Differences with the apoenzyme 


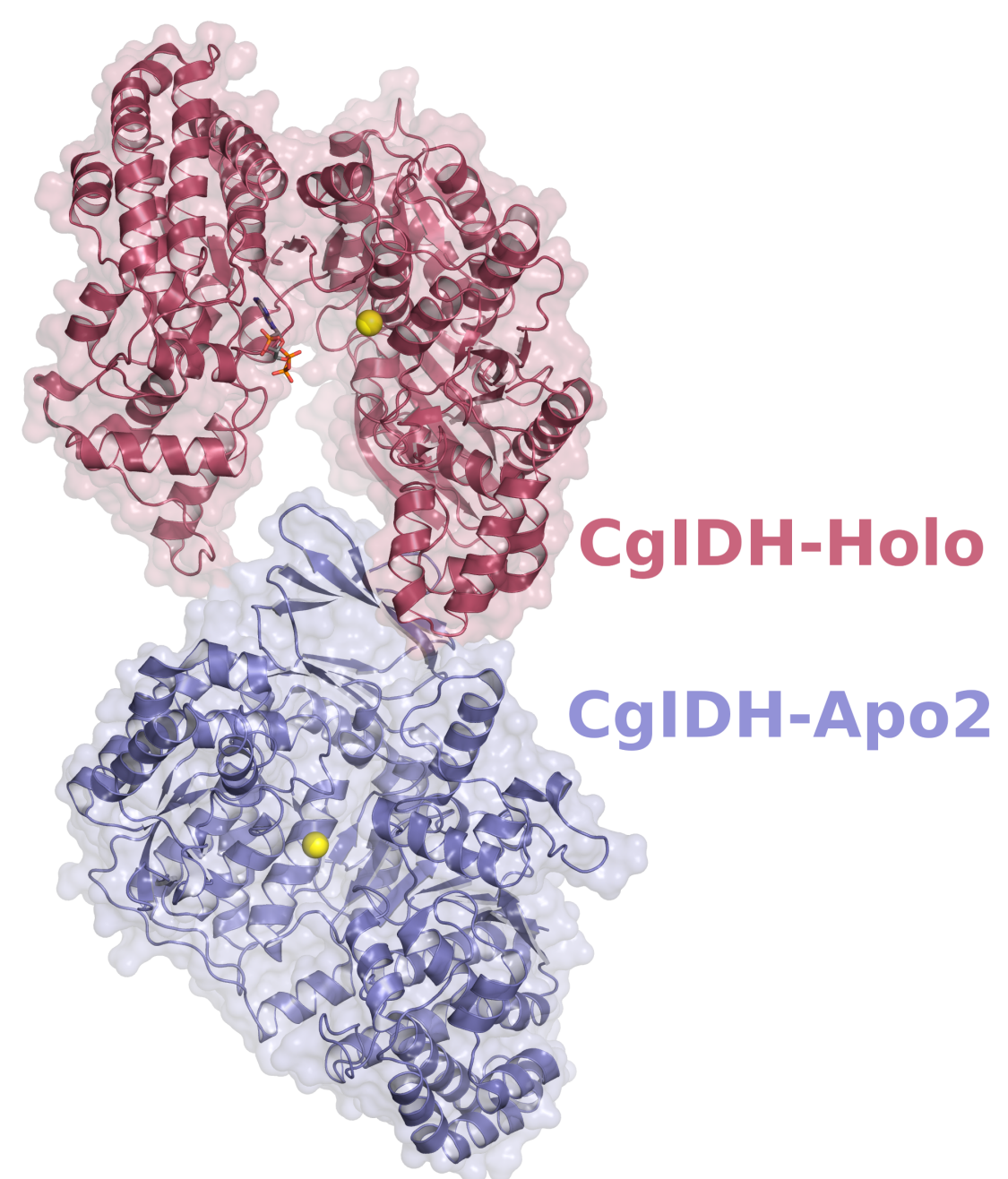

Figure 2.4: Asymmetric unit with holo- and apoenzyme models of the monomeric IDH and their relationship in the crystal. Holoenzyme (CgIDH-Holo, maroon), apoenzyme (CgIDH-Apo2, blue), $\mathrm{NADP}^{+}$(stick model in interdomain cleft of Holo form, standard colors) and $\mathrm{Mg}^{2+}$ (a yellow ball each in both molecules) are shown. Figure published in Sidhu et al. (2011); copyright International Union of Crystallography (IUCr); used with permission. 
chain will be pointed out where appropriate.

\subsubsection{Overall structure}

The overall structure of the Holo form is similar to the previously determined structures of monomeric IDHs CgIDH-Apo1 (Apo1), AvIDH-Iso and AvIDHHolo. It consists of two domains: a small Domain I (313 residues overall; residues 1-138 and 564-738 in the amino acid sequence) and a large Domain II (425 residues; 139-563) (Fig. 2.5). The $\mathrm{NADP}^{+}$-binding site lies in Domain I; the metal-binding site in Domain II. The N-and C-termini both lie in Domain I. The protein chain starts in Domain I, crosses over to form the complete Domain II before returning to form the rest of Domain I. The first cross-over point lies in a loop ( $\alpha 6-\beta 4$; residues $138-139)$, and the second inside a strand $\beta$ strand ( $\beta 23$; 563-564). A 10 -stranded $\beta$ sheet consisting of mostly parallel strands $(\beta 1-\beta 4$, $\beta 15$, and $\beta 20-\beta 24)$ spans the two domains.

Overall, there are $26 \alpha$ helices $(\alpha 1-\alpha 26)$ and $24 \beta$ strands ( $\beta 1-\beta 24)$. Of these, $13 \alpha$ helices $(\alpha 1-\alpha 6, \alpha 20-\alpha 26), 4$ entire $\beta$ strands $(\beta 1-\beta 3, \beta 24)$ and part of one ( $\beta 23)$ lie in Domain I; $13 \alpha$ helices $(\alpha 7-\alpha 19), 19$ entire $\beta$ strands $(\beta 4-\beta 22)$ and part of one $(\beta 23)$ lie in Domain II. All of the Doman I $\beta$ strands belong to the interdomain $\beta$ sheet. A pseudo-twofold axis relates parts of Domain II, as previously described for other monomeric IDHs (Yasutake et al., 2002; Yasutake et al., 2003; Imbayashi et al., 2006).

\subsubsection{Comparison of CgIDH Holo, Apo1 and Apo2 forms}

The Holo and the previously described Apo1 forms of the enzyme adopt relatively similar open conformations while the Apo 2 form in the current structure adopts a half-open conformation. The inter-domain rotation occurs at two hinge points. Results of comparisons between CgIDH-Holo with the Apo1 and Apo2 forms is summarized in a part of Table 2.3. The relative positions of the two domains in the Holo form differ from those in Apo1 and Apo2 by a rotation of approximately $1^{\circ}$ (Fig. 2.6a) and $13^{\circ}$ (Fig. 2.6b), respectively. The domains of the Holo form are superimposable on equivalent domains of Apo1 and Apo 2 forms with $C_{\alpha}$ RMSDs of $0.41 \AA$ and $0.43 \AA$ for Domains I, and $0.26 \AA$ and $0.39 \AA$ for Domains II.

To find out if the interdomain rotation was focused in a particular part, the structure was analyzed using the program DYNDOM (Hayward and Berendsen, 1998). Two mechanical hinges were located. The first is at the position of residues Glu138 and Gly139 (N Hinge) that lie in the loop $\alpha 6-\beta 4$; the second at Ser563 and Val564 (C Hinge), which lie in the middle of strand $\beta 23$ (Leu562-565), which in turn is the central strand in the 10-strand $\beta$-sheet that spans the two domains. Of the hinge residues, Gly139 and Ser563 are strictly conserved in representative 
Table 2.1: Data collection and refinement statistics, IDH

\begin{tabular}{|c|c|}
\hline PDB ID & $3 \mathrm{MBC}$ \\
\hline Space group & $\mathrm{C} 2$ \\
\hline \multicolumn{2}{|l|}{ Unit cell parameters } \\
\hline $\mathrm{a}(\AA)$ & 128.8 \\
\hline $\mathrm{b}(\AA)$ & 52.7 \\
\hline$c(\AA)$ & 236.4 \\
\hline$\beta\left({ }^{\circ}\right)$ & 103.4 \\
\hline \multicolumn{2}{|l|}{ Data Collection } \\
\hline Wavelength $(\AA)$ & 0.9793 \\
\hline Resolution range $(\AA)$ & $19.7-1.9(1.95-1.90)$ \\
\hline No. of reflections measured & 438308 \\
\hline No. of unique reflections & 119827 \\
\hline Redundancy & $3.66(3.25)$ \\
\hline $\mathrm{R}_{\text {meas }}$ & $0.103(0.364)$ \\
\hline Completeness (\%) & $97.8(85.4)$ \\
\hline Mean $I / \sigma(I)$ & $9.94(3.54)$ \\
\hline \multicolumn{2}{|l|}{ Refinement } \\
\hline Resolution limit $(\AA)$ & 19.7-1.90 \\
\hline $\mathrm{R}_{\text {free }}$ (5992 reflections) & 0.229 \\
\hline $\mathrm{R}_{\text {work }}$ & 0.189 \\
\hline Total no. of reflections (working set) & 113834 \\
\hline Solvent content $(\%)$ & 49.5 \\
\hline Number of molecules in the asymmetric unit & 2 \\
\hline No. of non-H atoms refined & 12027 \\
\hline No. of water molecules refined & 794 \\
\hline Mean B factor, protein atoms $\left(\AA^{2}\right)$ & 38.2 \\
\hline Mean B factor, backbone atoms $\left(\AA^{2}\right)$ & 37.0 \\
\hline Mean B factor, side-chain atoms $\left(\AA^{2}\right)$ & 39.5 \\
\hline Mean B factor, $\mathrm{NADP}^{+}\left(\AA^{2}\right)$ & 43.6 \\
\hline Mean B factor, water atoms $\left(\AA^{2}\right)$ & 36.3 \\
\hline Mean B factor, protein atoms, $\mathrm{A}$ chain $\left(\AA^{2}\right)$ & 26.3 \\
\hline Mean B factor, protein atoms, B chain $\left(\AA^{2}\right)$ & 50.2 \\
\hline \multicolumn{2}{|l|}{ RMSDs from ideal geometry } \\
\hline Bond length $(\AA)$ & 0.012 \\
\hline Bond angles $\left(^{\circ}\right)$ & 1.3 \\
\hline \multicolumn{2}{|l|}{ Ramachandran statistics } \\
\hline Favored region $(\%)$ & 97.9 \\
\hline Allowed region $(\%)$ & 2.1 \\
\hline Outlier region $(\%)$ & 0.0 \\
\hline
\end{tabular}

Values in parentheses indicate the outermost resolution shell. Number of atoms represent the sum of occupancies; B factors are the occupancy-weighted means. Table adapted from Sidhu et al. (2011). 

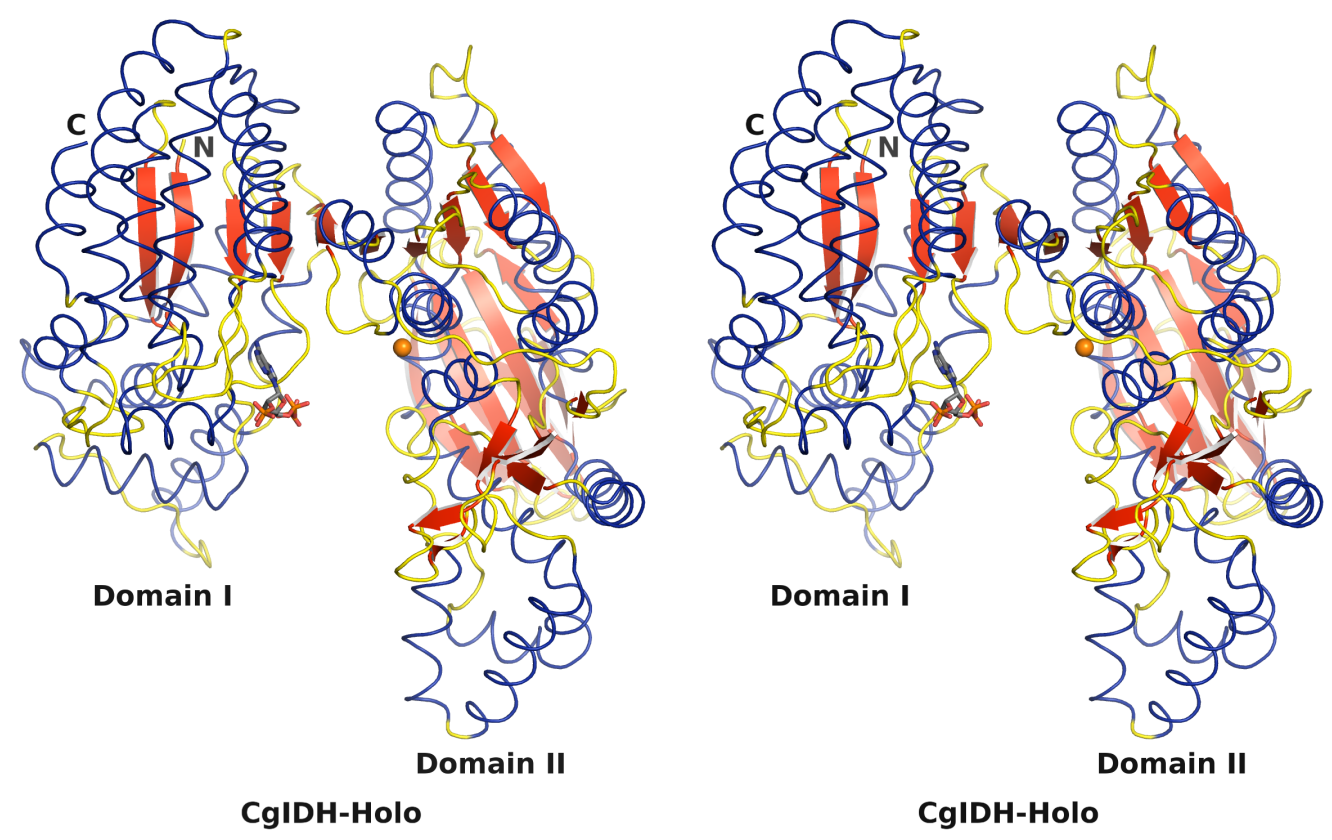

Figure 2.5: Stereo view of the location of secondary structure elements in the CgIDH Holo form. The two domains are labeled (Domain I and II). Helices (blue), $\beta$ strands (red), loops (yellow). The $\mathrm{N}$ - and $\mathrm{C}$-terminus are labeled ( $\mathrm{N}$ and $\mathrm{C}$, respectively; upper left). Coenzyme (stick model in standard colors) binds to Domain I; $\mathrm{Mg}^{2+}$ (orange ball) to Domain II. Figure published in Sidhu et al. (2011); copyright IUCr; used with permission. 


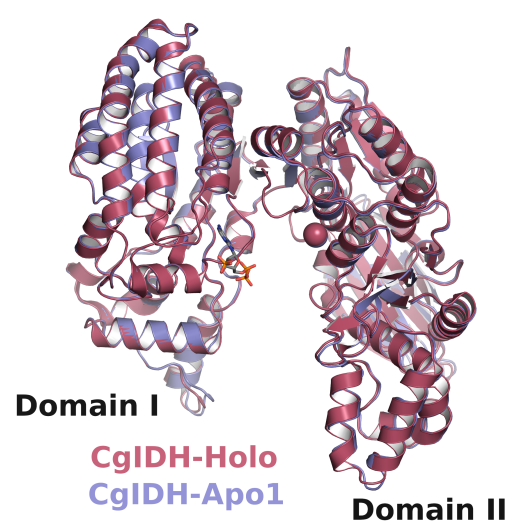

(a) CgIDH-Holo and -Apo1

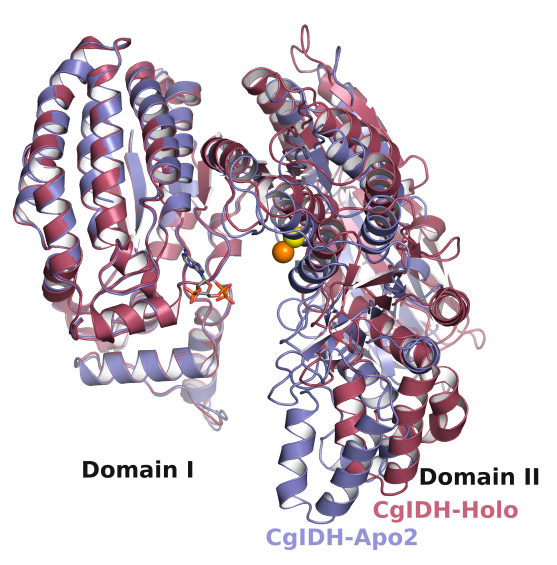

(b) CgIDH-Holo and -Apo2

Figure 2.6: Comparison of CgIDH-Holo and -Apo1 and -Apo2 forms. The Holo and Apo2 forms are present in the same crystal. Shown are: CgIDH-Holo (maroon); -Apo1 (blue) and -Apo2 (blue); $\mathrm{Mg}^{2+}$ bound to Holo (maroon ball) and Apo2 (orange ball). Fig. in (b) published in Sidhu et al. (2011); copyright IUCr; used with permission.

monomeric IDH sequences (described further in the Methods section). The hydrogen bonding pattern in the sheet is in general conserved over the hinge motion observed.

\subsubsection{Comparison of CgIDH and AvIDH}

Large open/close conformational differences are observed between the Holo form of $\mathrm{CgIDH}$ and the two previously determined AvIDH structures, with the $\mathrm{CgIDH}$ structure being in a more open conformation than AvIDH (Fig. 2.7). The N Hinge lies within the isocitrate-binding site. Results of comparisons of $\mathrm{CgIDH}-\mathrm{Holo}$ with AvIDH structures are summarized in Table 2.3. The relative positions of the two domains in the Holo form differ from those in AvIDH-Holo and AvIDH-Iso by a rotation of approximately $36^{\circ}$ and $24^{\circ}$, respectively. The domains of the Holo form are superimposable on equivalent domains of these AvIDH structures with $\mathrm{C}_{\alpha}$ RMSDs of $1.4 \AA$ and $0.76 \AA$ for Domains I, respectively, and $1.5 \AA$ and $1.3 \AA$ for Domains II.

\subsubsection{The $\mathrm{N}$ Hinge and the isocitrate binding site}

The 9 putative isocitrate binding residues (identified based on AvIDH-Iso, with a distance cutoff of $3.5 \AA$ ) are strictly conserved in the monomeric IDH representative sequences. For CgIDH, these residues are: Ser130, Asn133 and Arg137 in 


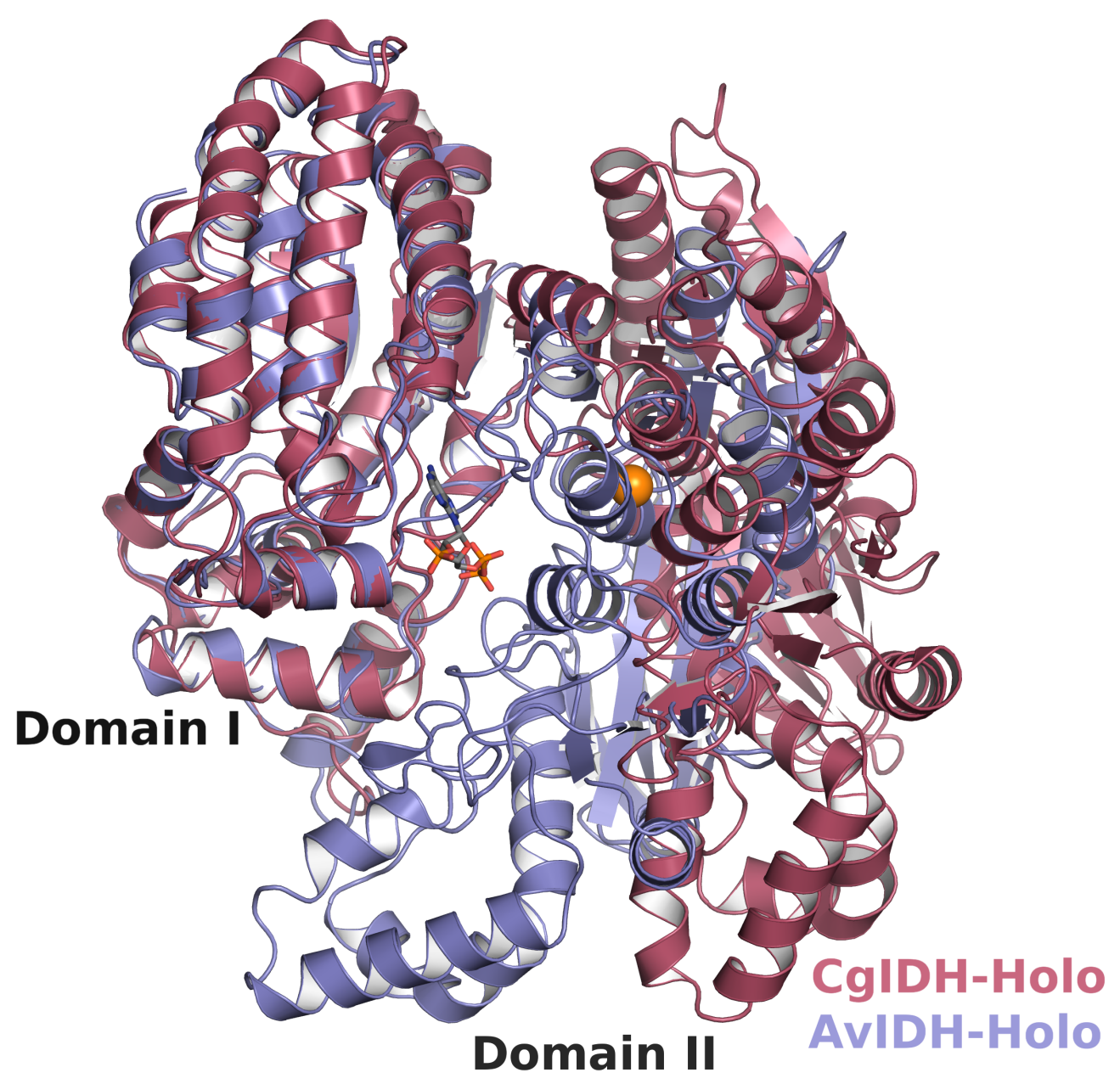

Figure 2.7: CgIDH-Holo and AvIDH-Holo

Figure 2.8: Comparison of CgIDH-Holo and AvIDH-Holo. In the superposition, the distance between Domains II of the CgIDH and AvIDH holo forms is up to 34 $\AA$, with CgIDH-Holo in an open conformation and AvIDH-Holo in a closed one. Figure published in Sidhu et al. (2011); copyright IUCr; used with permission. 
Table 2.2: Comparison of CgIDH-Holo with other monomeric IDHs.

\begin{tabular}{lcccc}
\hline & CgIDH & CgIDH & AvIDH & AvIDH \\
& -Apo1 & -Apo2 & -Holo & -Iso \\
Domain I RMSD / A & 0.41 & 0.43 & 1.4 & 0.76 \\
Domain II RMSD / A $^{-1}$ & 0.26 & 0.39 & 1.5 & 1.3 \\
Angle / $^{\circ}$ & 1 & 13 & 36 & 24
\end{tabular}

Superpositions and RMSDs are for $\mathrm{C}_{\alpha}$ atoms. Angle stands for the interdomain rotation angle relative to that of CgIDH-Holo. For Domains I, RMSDs listed are for 310 of 313 Holo $\mathrm{C}_{\alpha}$ atoms; for Domains II for 422 (for comparison with CgIDH-Apo2) or 423 (all except CgIDH-Apo2) of 425 atoms. For Domain II RMSD calculations against AvIDH, CgIDH-Holo residues 139-305 and 306-563 were overlaid, respectively, on AvIDH residues 141-307 and 310-567.

Domain I, and Arg143, Lys253, Asp346, Tyr416, Arg543 and Asp544 in Domain II. Fig. 2.8 shows the close relationship of the isocitrate binding site with the $\mathrm{N}$ Hinge in a hypothetical structure in which isocitrate- $\mathrm{Mn}^{2+}$ is bound to $\mathrm{CgIDH}-$ Holo based on a Domain II overlay with AvIDH-Iso. The isocitrate binding site (e.g. residues Arg137 and Arg143) flanks the N Hinge (Glu138/Gly139) both in sequence and 3-D structure. Residues from both domains contribute to the site.

To achieve an appreciation of possible domain motions in monomeric IDHs, $\mathrm{CgIDH}-\mathrm{Holo}$ and -Apo2 forms in the current structure and AvIDH-Iso were all superimposed on their Domains II. All three structures appear to diverge from each other in the two hinge regions (Fig. 2.9). The divergence point appears to be more focused for the $\mathrm{N}$ Hinge, which lies in a loop, and less so for the $\mathrm{C}$ Hinge, which is part of the interdomain $\beta$-sheet.

Domain motions are large and appear to lead to a significant opening and closing of the isocitrate binding site. If the CgIDH and AvIDH holoenzyme structures are superposed on Domains I, the extreme points of their Domains II are separated by as much as $33 \AA$ (distance between Leu305 $\mathrm{C}_{\alpha}$ in $\mathrm{CgIDH}$ and the structurally equivalent Leu307 $\mathrm{C}_{\alpha}$ in AvIDH). The entry to the isocitrate binding site is relatively widely accessible in the open conformation ( $\mathrm{CgIDH}-\mathrm{Holo}$ ) but closed in the closed conformation (AvIDH-Holo). For example, at what appears to be a narrow point in the access route to the binding site, the two domains are separated by $11.6 \AA$ in the open conformation but only $4.2 \AA$ in the closed one (Ser130 $\mathrm{C}_{\beta}$ to Met $256 \mathrm{C}_{\beta}$ distance in $\mathrm{CgIDH}-\mathrm{Holo}$ compared with the structurally equivalent Ser132 to Met258 distance in AvIDH-Holo). 


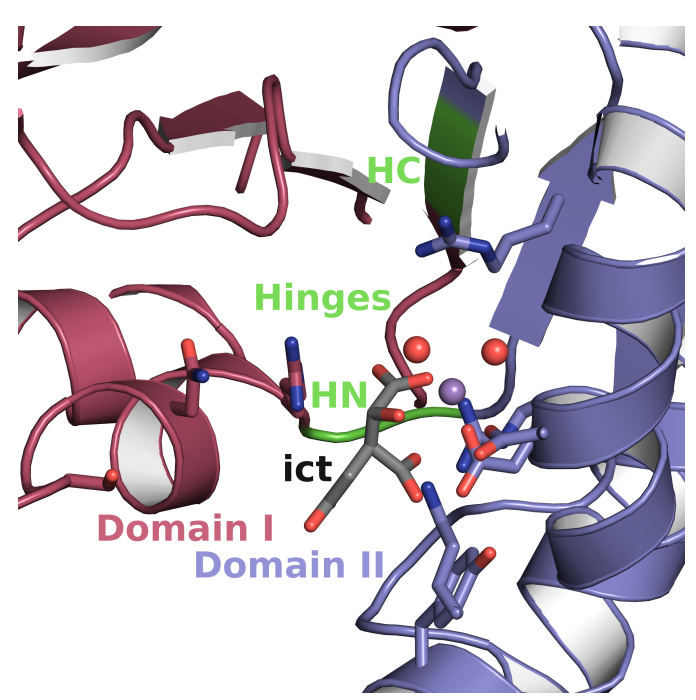

Figure 2.9: Hypothetical model of isocitrate in binding site at an interdomain hinge. Domain I (maroon), Domain II (blue); isocitrate (stick model; ict), putative isocitrate binding residues (stick models, oxygen red, nitrogen blue), divalent metal (blue ball), water oxygens (red balls); interdomain hinges (labeled as Hinges $\mathrm{HN}$ and HC in green). Figure published in Sidhu et al. (2011); copyright IUCr; used with permission.

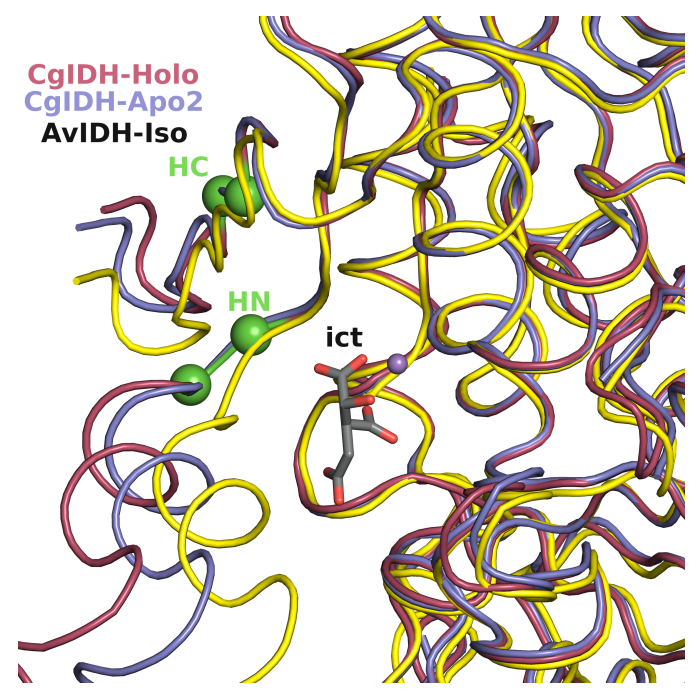

Figure 2.10: Hinge motion close to the isocitrate binding site. The models of CgIDH-Holo (maroon) and -Apo2 (blue) from the present structure and the previously described AvIDH-Iso (with the bound isocitrate and divalent metal cation) are superimposed on Domains II. Figure published in Sidhu et al. (2011); copyright IUCr; used with permission. 


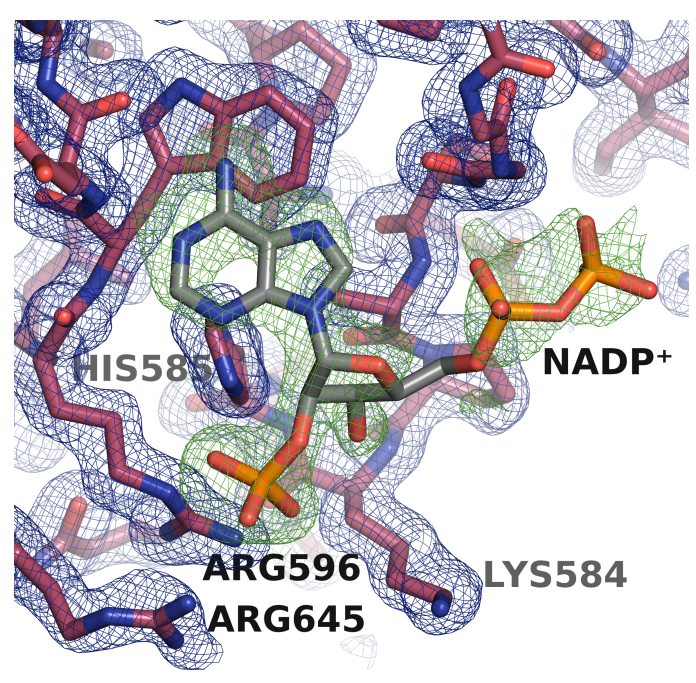

Figure 2.11: $\mathrm{NADP}^{+}$omit map. Electron density and stick model built into it (protein carbons maroon, the rest and $\mathrm{NADP}^{+}$in standard colors). The coenzyme and major residues seen to mediate coenzyme specificity are labeled. The map was calculated before the addition of the coenzyme part to the model. Figure published in Sidhu et al. (2011); copyright IUCr; used with permission.

\subsubsection{NADP $^{+}$binding}

The NADP ${ }^{+}$binding site lies in Domain I in the interdomain cleft. $\mathrm{NADP}^{+}$is bound in a conformation of the enzyme that leaves the nicotinamide half of the molecule highly solvent-exposed. Fig. 2.10 displays the electron density in the coenzyme binding site, including the omit density for the coenzyme. A clear variation in the quality of the electron density is seen from one end of the coenzyme to the other. Density for the adenosine- $2^{\prime}$-phosphomonoester part was observed to be well-formed with sharp features. B factors for this part of the molecule refined to low values (to a minimum of $13 \AA^{2}$, occupancy 0.9 ), approximately equivalent to those of neighboring protein atoms. On the other hand, density corresponding to the $5^{\prime}$-diphosphate moiety was observable but of a more disordered quality, and the coenzyme B factors were higher (up to $71 \AA^{2}$ ) than for the neighboring protein atoms. No clear density could be spotted for nicotinamide and the ribose it is attached to. These moieties are not part of the structure model.

Three surface loops and the N-terminal end of an $\alpha$ helix contribute directly binding residues to the site (Fig. 2.11). Binding of the adenine moiety of the coenzyme is stabilized through $\pi$-stacking interactions and direct and water-mediated hydrogen bonds. The ribose makes only one indirect, water-mediated hydrogen bond. The diphosphate backbone forms less well-defined direct and water- 
mediated connections. Binding of the $2^{\prime}$-phosphate moiety of the coenzyme is, in contrast, very well defined, with major contributions from salt bridges and direct hydrogen bonds with 3 positively charges residues ( 2 arginines and 1 histidine), and a minor contribution from and indirect hydrogen bond with a lysine.

The secondary structure elements involved are Helix $\alpha 20$, and loops $\beta 24-\alpha 20$, $\alpha 20-\alpha 21$ and $\alpha 22-\alpha 23$. The residues Gly580, His585, Arg596, Trp597, Asp598 and Arg645 are involved in binding directly, while Thr576, Gly579, Ala582, Lys584, Gly601 and Glu602 contribute water-mediated interactions.

All 3 nitrogen atoms on the edge of the adenine ring opposite from the $\mathrm{N}$ glycosidic bond are involved in direct or water-mediated hydrogen bonds. Apparent $\pi$-stacking interactions occur amongst an aromatic triad formed by the adenine ring, Trp597 and His585. The angle between the ring plane of adenine and that of Trp597 and His585 is approximately $25^{\circ}$ and $85^{\circ}$, respectively, and that between the latter two rings $75^{\circ}$. The adenine ring is set in an offset arrangement in relation to Trp597 and His585 rings.

The 3'-hydroxyl group of ribose is stabilized through a water-mediated hydrogen bond with the backbone amide of Lys584. The adenine ring and the endocyclic oxygen of ribose are mutually related by an anti conformation (Klyne and Prelog, 1960) around the N-glycosidic bond. The ribose furanoid ring has an approximately half chair $\mathrm{C}^{\prime}$-endo/C2'-exo conformation (Sundaralingam, 1969). The diphosphate backbone of $\mathrm{NADP}^{+}$forms direct and indirect hydrogen bonds with backbone amides of Gly580 and Ala582.

The 2 -phosphate group forms salt bridges and direct hydrogen bonds with the side chains of His585, Arg596, Arg645 and a water-mediated hydrogen bond to Lys584.

The NADP ${ }^{+}$-binding region of the Holo and Apo1 forms has a very similar structure. In contrast, the Holo and Apo2 forms of the enzyme in the same asymmetric unit in the current structure display significant differences between each other. In Apo2, the coenzyme binding site is exposed even in the half-open conformation of the enzyme such that it allows close approach to a symmetry-related molecule in the crystal, apparently disrupting binding: The side chain of the crucial coenzyme binding residues Arg596 forms a salt bridge and double hydrogen bond with the side chain of Asp506 from this symmetry-related molecule.

The side chain of Arg645 appears to occupy part of the space where the coenzyme is in the Holo form. The side chains of Arg645 and Arg596 also form potential salt bridges and hydrogen bonds with a disordered side chain of Glu508.

\subsection{8 $\mathrm{Mg}^{2+}$ binding}

The divalent metal ion binding site lies in Domain II. The residues (demonstrably or putatively) involved are strictly conserved in the sequences of all 21 represen- 


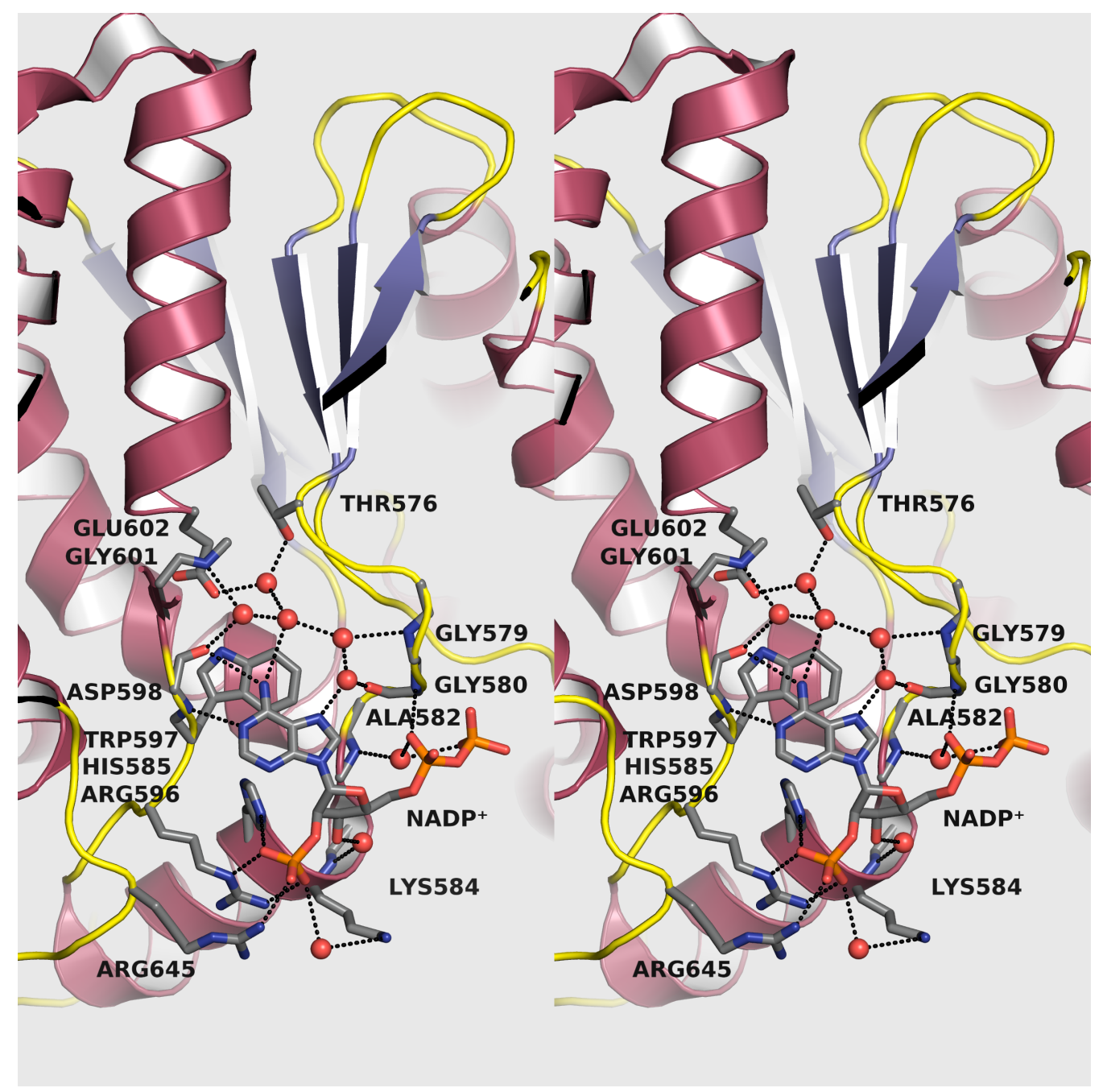

Figure 2.12: Stereo view of $\mathrm{NADP}^{+}$-binding interactions. $\mathrm{NADP}^{+}$and protein residues involved in coenzyme binding interactions are shown as stick models in standard colors (carbon gray, oxygen red, nitrogen blue, phosphorus orange); oxygen atoms of water molecules mediating binding as red balls. Helices (maroon), $\beta$ strands (blue), loops (yellow). Figure published in Sidhu et al. (2011); copyright IUCr; used with permission. 
tative monomeric IDHs.

Both Holo and Apo 2 forms of the enzyme in the asymmetric unit of the current structure each have a $\mathrm{Mg}^{2+}$ ion bound at this site. The cation is hexa-coordinated in an approximately octahedral manner to oxygen ligands. These ligands are Asp544 (bidentate binding with the backbone carbonyl $\mathrm{O}$ and sidechain carboxylate), a sidechain carboxylate oxygen each from Asp346 and Asp548, and two water oxygens.

An overlay of the Holo form with AvIDH-Iso shows that, in the latter, 1) $\mathrm{O} 2$ and $\mathrm{O} 7$ oxygens of isocitrate occupy the water sites in the former; 2) two waters replace the backbone carbonyl of Asp544 and the side chain carboxylate of Asp548; and 3) the positions of the side chain oxygens of Asp346 and Asp544 are similar to those in the latter.

\subsection{Discussion}

The crystal structure of the monomeric isocitrate dehydrogenase from Corynebacterium glutamicum (CgIDH) co-crystallized with its coenzyme $\mathrm{NADP}^{+}$and cofactor $\mathrm{Mg}^{2+}$ establishes the molecular basis of coenzyme and cofactor binding in this highly isocitrate- and $\mathrm{NADP}^{+}$-specific enzyme. One of the 2 enzyme molecules in the asymmetric unit is in the coenzyme-bound, holoenzyme form (CgIDH-Holo). The other molecule is in the coenzyme-unbound, apoenzyme form (CgIDH-Apo2). An apoenzyme structure for the enzyme has been described previously (CgIDH-Apo1; Imabayashi et al., 2006; PDB accession code 2B0T). Each enzyme molecule has $\mathrm{Mg}^{2+}$ ion bound at the putative cofactor binding site.

The coenzyme is bound at the putative coenzyme binding site. It lies significantly solvent-exposed in a highly open conformation of the enzyme. Approximately a third of the coenzyme is well ordered, another third less well ordered, and another third apparently disordered. Crystal contacts disrupt coenzyme binding in the apoenzyme.

The enzyme consists of two domains. Two hinge points were identified at the junction of the two domains. The conformations of the holo- and apoenzyme molecules in the same crystal differ by a hinge rotation of $13^{\circ}$, indicating hinge bending that had previously been suggested for this enzyme (Yasutake et al., 2003; Imabayashi et al., 2006). One of the hinge points lies withing the isocitrate binding site, suggesting a substrate-induced conformational change in the enzyme.

\subsubsection{Cell parameters}

The enzyme crystallized in the same space group, C2, as for the Apo1 structure described previously. The solvent content is also similar. However, in this case, there are 2 molecules in the asymmetric unit rather than 1 , which was the case for 
Apo1. The unit cell in this crystal is thus approximately twice as large as that for the previous structure, essentially through the doubling of the unit cell dimension c (236.4 $\AA$ in contrast with $124.0 \AA$ A before).

\subsubsection{Overall structure}

Conformational change upon coenzyme binding was suggested for this enzyme on the basis of a change in the intensity of fluorescence upon coenzyme addition (Imabayashi et al., 2006). This would appear to be consistent with the different conformations of the holo- and apoenzyme in the same crystal in the present structure. The two conformations differ by a hinge bending of $13^{\circ}$. However, the conformation of the Holo form in the present crystal is highly similar to that of the Apo1 form in the previously described apoenzyme structure for this enzyme under quite similar crystallization conditions. The interpretation of the overall conformation and its relationship to coenzyme binding is complicated by a couple of factors. First, as mentioned, this enzyme has a highly flexible hinge. This is also suggested by the $36^{\circ}$ hinge rotation between the holoenzyme structures of $\mathrm{CgIDH}$ and its homologue AvIDH.

Second, an examination of the asymmetric unit shows that the two molecules lie with their Domains II inserted into each other's interdomain cleft (Fig. 2.4). This would suggest that changes in conformation in the enzyme could also be induced by crystal packing. This caveat has been suggested previously for AvIDHHolo. Crystal structure and small-angle X-ray scattering results in a more distant homologue, the dimeric IPMDH from Thermus thermophilus (Kadono et al., 1995), also pointed to this interpretation in that enzyme. Thus, ascribing observed overall conformation changes in crystal structure to ligand binding in an apparently flexible enzyme is considered unwarranted for the present structure.

\subsubsection{Hinge bending}

In AvIDH, which is a homologue of CgIDH, hinge movements have been described based on a comparison of two different crystal structures of the enzyme (AvIDH-Holo and AvIDH-Iso). These movements occurred in 3 groups of residues that were part of 2 loops, in 11 residues overall (residues 137-138, 142-144 and 560-565, AvIDH numbering; Yasutake et al., 2003). Isocitrate was found to bind to both domains. Hence, its role in fixing the two domains was suggested.

In $\mathrm{CgIDH}$, since the enzyme amino acid chain forms part of Domain I and then the whole of Domain II before crossing back to form the rest of Domain I, at least two inter-domain hinges were predicted. The prediction could be confirmed for molecules that were part of the same crystal, indicating that the conformational changes take place under a single set of solution conditions and are not an artifact 
of different solution conditions. The Holo and Apo2 forms in the present crystal display a $13^{\circ}$ rigid body hinge bending with respect to each other. The hinges could be pinpointed to two groups, each consisting of 2 residues.

Putative isocitrate binding residues are highly conserved in monomeric IDHs. In $\mathrm{CgIDH}$, the N Hinge (Glu138-Gly139) is flanked in sequence and 3-D structure, by putative isocitrate-binding residues belonging to both domains. A model linking isocitrate binding and conformational change could thus be proposed: The binding of a small molecule (the substrate isocitrate) close to and on both sides of a pinpoint mechanical hinge is partly responsible for the large-scale change in enzyme conformation from an open to a closed one. The structures CgIDH-Holo and AvIDH-Iso approximately capture these two conformations, respectively, that differ by a $24^{\circ}$ hinge movement.

Four different conformations differing by $9-13^{\circ}$ in hinge movements have been observed for monomeric IDHs in the 4 structures solved to date, corroborating the suggestion (Yasutake et al., 2003; Imabayashi et al., 2006) that these enzymes are highly flexible. The flexibility appears to be focused at the interdomain hinge regions. Hinge movements have been described in other proteins (see Gerstein et al., 1994, for a review), including EcIDH (Gonçalves et al. and references therein).

\subsubsection{Active site access}

In AvIDH-Holo, the only previously described holoenzyme structure for any monomeric IDH, the enzyme was in a closed, apparently inactive conformation in which access for the substrate isocitrate to the isocitrate binding site was blocked by the coenzyme. Lack of sufficient space for the substrate in the binding site of the flexible enzyme was ascribed to crystal packing forces. This interpretation appears plausible also on the basis of results from kinetics experiments that indicated that the binding of the first substrate should not significantly change the binding of the second for the random order binding in the forward direction (Wicken et al., 1972). The situation appears to be similar in EcIDH, where the binding of $\mathrm{NADP}^{+}$does not influence the $\mathrm{K}_{m}$ for isocitrate (Dean and Koshland, 1993).

The present structure shows that $\mathrm{NADP}^{+}$-bound enzyme can still retain a conformation in which the substrate binding site is accessible to isocitrate: $\mathrm{CgIDH}$ is in a large, $36^{\circ}$ hinge-open state as compared with AvIDH-Holo. It appears plausible that the present conformation is close to a physiological one. Indeed, the putative isocitrate binding residues lie too distant from each other. Productive isocitrate binding would be expected to favor a closed conformation of the enzyme, which then is presumed to be the catalytic conformation comparable to the one seen in AvIDH-Iso. A conformation intermediate between the open and closed ones is displayed by $\mathrm{CgIDH}-\mathrm{Apo} 2$ in the present structure. The overall 
description of conformational change upon isocitrate binding is consistent with previous results in $\mathrm{CgIDH}$ that showed changes in fluorescence upon the addition of isocitrate (Imabayashi et al., 2006).

\subsubsection{Possible regulatory and biological implications}

Different modes of regulation have been described in different IDHs. The homodimeric Escherichia coli IDH (EcIDH) is enzymatically inhibited by reversible phosphorylation of an active site serine residue (Ser113) (Garnak and Reeves, 1979; LaPorte and Koshland, 1982; Hurley et al., 1990; Zheng and Jia, 2010). Hetero-oligomeric $\mathrm{NAD}^{+}$-IDHs in eukaryotic are allosterically regulated (Taylor et al., 2008). Human cytosolic $\mathrm{NADP}^{+}$-IDH has been suggested to be selfregulated (Xu et al., 2004). In addition, concerted inhibition by glyoxylate and oxaloacetate has also been reported in dimeric and monomeric IDHs (Shiio and Ozaki, 1968; Ochiai et al., 1979; Leyland and Kelly, 1991), including CgIDH (Eikmanns et al., 1995). They have also been shown to be concerted competitive inhibitors of IDHs with respect to isocitrate binding (Shiio and Ozaki, 1968). Although the need for the inhibition of CgIDH appears to be necessary, the mechanism of inihibition is unclear (Reinscheid et al., 1994; Eikmanns et al., 1995). Since isocitrate and divalent metal-binding residues are highly conserved between monomeric and dimeric IDHs (Yasutake et al., 2002), it is speculated that the concerted inhibitors bind to similar residues also in monomeric IDHs, that is, in competition with isocitrate. Large hinge bending appears to accompany isocitrate binding (e.g. the $24^{\circ}$ hinge bending between CgIDH-Holo and AvIDH-Iso). Whether a similar movement accompanies the binding of the inhibitors may be testable in solution by small-angle X-ray scattering (SAXS).

It has been suggested (Banerjee et al., 2004) that IDHs from M. tuberculosis could serve as potential drug targets against $\mathrm{TB}$, which is a leading cause of infectious disease mortality. If this suggestion is confirmed, glyoxylate and oxaloacetate and their analogues could be explored as candidate drugs.

\subsection{6 $\mathrm{NADP}^{+}$binding}

Residues that directly bind $\mathrm{NADP}^{+}$in $\mathrm{CgIDH}$ belong to an $\alpha$ helix and three loops, which is different from the Rossmann fold (Rossmann et al., 1974) whose typical motif is $\beta \alpha \beta \alpha \beta$. In characterized monomeric IDHs from $C$. glutamicum, A. vinelandii, $C$. maris, $R$. vannielii, and $V$. parahaemolyticus, coenzyme-binding residues are in general strictly conserved. The aromatic rings of adenine, Trp597 and His585 make $\pi$-stacking interactions with each other. Adenine and Trp597 rings come together in offset and T-shaped (edge-on) arrangements. The interaction of His585 with the other two aromatics is approximately of the T-shaped 
type. Both these components are consistent with an attractive interaction geometry (Hunter and Sanders, 1990). Coenzyme binding in $\mathrm{CgIDH}$ is in general similar to that observed in AvIDH. However, the present structure is based on data to $1.9 \AA$ in contrast with the AvIDH one, where the data resolution was only up to $3.2 \AA$. In the $\mathrm{CgIDH}$ structure, a network of waters is shown to play an important role in anchoring the outer edge of the adenine moiety to the binding site through watermediated hydrogen bonds. The coenzyme beyond the ribose on the diphosphate side is less well ordered. It appears to form relatively few interactions with the protein in CgIDH. A similar observation was made in AvIDH-Holo and EcIDH (Bolduc et al., 1995; PDB ID 1IDE).

\subsubsection{NADP $^{+}$specificity}

$\mathrm{NADP}^{+}$differs from $\mathrm{NAD}^{+}$in having a $2^{\prime}$-phosphate group, which is absent in the latter. Thus, recognition of this group by the enzyme would appear to be important in achieving the exquisite specificity of this enzyme for its coenzyme $\mathrm{NADP}^{+}$.

The phosphate group is surrounded by 3 well-ordered side chains, two of them arginines and one histidine, that form salt bridges and hydrogen bonds. These side chains are His585, Arg596 and Arg645. A fourth residue, Lys584, forms a watermediated hydrogen bond.

Lys584 and His585 lie close to the N-terminal end of Helix $\alpha 20$. Thus, the helix dipole moment could contribute in their phosphate-binding interactions (Wierenga et al., 1985). These two residues were predicted to be involved in phosphate binding by Chen and Yang (2000).

His585, Arg596 and Arg645 are highly conserved in representative IDHs. An interesting one of two excpetions is the putative monomeric IDH from Ralstonia eutropha (ReIDH). In it, the residue at the position equivalent to Arg645 is a proline (Pro652, ReIDH numbering). When expressed in E. coli, ReIDH lacked IDH activity (Wang et al., 2003). Although unproven, the switch could be a possible reason for the inactivity.

Chen and Yang (2000) suggested a divergent evolution for CgIDH and EcIDH, which prefers $\mathrm{NADP}^{+}$to $\mathrm{NAD}^{+}$by a factor of 7,000. In EcIDH, NADP ${ }^{+}$specificity is mainly due to Arg395, Tyr345, Tyr391 and Arg292' (the prime denotes the second subunit in the dimer) (Hurley et al., 1991; Chen et al., 1995). While the two tyrosines present in EcIDH are replaced in CgIDH by the His and Lys residues from an $\alpha$ helix, there are many similarities between the two enzymes. Structurally equivalent residues in EcIDH/CgIDH include the following: Arg395/Arg596, Arg292'/Arg645, Gly340/Gly580, Ala342/Ala582 and Lys344/Lys584.

Overall, the structure appears to support a common ancestor for the two enzymes.

A monomeric IDH that is not highly specific for $\mathrm{NADP}^{+}$is that Rhodomicro- 
bium vannielii (RvIDH), which prefers $\mathrm{NADP}^{+}$by a factor of 400 (Leyland and Kelly, 1991). It shares a high sequence similarity with $\mathrm{CgIDH}$. The three residues directly interacting with the $2^{\prime}$-phosphate are strictly conserved between the two. This suggests that as yet undetermined factors may play an important role in determining specificity. These could include "second layer" residues (Hurley et al., 1996) that interact with the $2^{\prime}$-phosphate via "first layer" residues, long-range interactions (Chen et al., 1997a), and molecular dynamics that may not be captured in the time-averaged picture offered by the crystal structure.

$\mathrm{CgIDH}$ binds the coenzyme with a high specificity in a relatively solventaccessible site. This appears to be consistent with the high specificity and performance achieved by the enzyme.

\subsubsection{Nicotinamide binding and disorder}

The nicotinamide moiety of the coenzyme participates directly as an electrontransferring group in enzymatic reactions. Different enzymes appear to employ different modes of binding for the nicotinamide part in the holoenzyme. In one mode, it is bound by the enzyme itself. In another, the enzyme binds a ligand that then forms at least part of the binding site for it. In the former mode, the nicotinamide may be ordered in the crystal structure. In the latter, it may be ordered or disordered depending on whether the binding site is present.

An ordered nicotinamide has been observed in many holoenzymes, including IDHs and AvIDH. AvIDH displays a high turnover number of $930 \mathrm{~s}^{-1}$ at $37{ }^{\circ} \mathrm{C}$ (Barrera and Jurtshuk, 1970) and $250 \mathrm{~s}^{-1}$ at $25^{\circ} \mathrm{C}$ (Wicken et al., 1972). For comparison, the turnover rate of CgIDH and EcIDH is $87 \mathrm{~s}^{-1}$ and $81 \mathrm{~s}^{-1}$, respectively $\left(21^{\circ} \mathrm{C}\right.$; Chen and Yang, 2000). AvIDH has been proposed to bind the nicotinamide mononucleotide moiety such that, upon the binding of the substrate, the reaction can take place without further time delay (Yasutake et al., 2003). The enzyme (Glu87) has been suggested to bind nicotinamide also in IPMDH from Thermus Thermophilus (Dean and Dvorak, 1995).

A disordered nicotinamide nucleotide, with or without disorder of the second 5'-phosphate, of the coenzyme has been observed in EcIDH crystal structures for wild-type (Hurley et al., 1991, PDB accession code 9ICD; Stoddard and Koshland, 1993; Stoddard et al., 1998, PDB accession code 1BL5) and mutant enzyme (Bolduc et al., 1995; Hurley et al., 1996, PDB code 1ISO) in the absence of the substrate isocitrate. However, when isocitrate was simultaneously present, electron density corresponding to the whole $\mathrm{NADP}^{+}$molecule was observed (Stoddard et al., 1993; Bolduc et al., 1995). The enzymatic residue Ser113 was found to form a crucial hydrogen bond with the $\gamma$-carboxylate of isocitrate, stabilizing it (Dean et al., 1996). The carboxylate then bound the positively charged nicotinamide (PDB accession code 1IDE; Bolduc et al., 1995), plausi- 
bly aligning it in hydride transfer.

$\mathrm{CgIDH}$ has been previously found to show a similar substrate specificity towards substrate analogues carrying different substituents at a position equivalent to the $\gamma$ carbon of isocitrate (Chen and Yang, 2000). Indeed, in comparison with with EcIDH, CgIDH was more specific towards isocitrate by an order of magnitude. It was proposed that this was due to the $\gamma$-carboxylate of the substrate forming the binding site for nicotinamide in this enzyme as in EcIDH.

This model offers one explanation for there being no observable electron density corresponding to the nicotinamide nucleoside (i.e., nicotinamide and the adjacent ribose) in the present structure. However, other possibilities cannot be ruled out. First, it is possible that the marked solvent exposure of the coenzyme bound to an open conformation of the enzyme is partly responsible. For comparison, the coenzyme bound to a closed conformation of the enzyme in AvIDH was found to be ordered.

Second, partial decomposition of the coenzyme also appears plausible. At higher $\mathrm{pH}\left(\mathrm{pH}\right.$ less than 14), the related coenzyme $\mathrm{NAD}^{+}$decomposes by and large through the base-catalyzed hydrolysis of the N-glycosidic bond. The products of this chemical reaction are adenosine-diphosphate-ribose (ADP-ribose) and nicotinamide (Oppenheimer, 1987). The rate is directly proportional to $\mathrm{pH}$ within the $\mathrm{pH}$ range of 11.5 to 9 . At lower $\mathrm{pH}$ ( $\mathrm{pH} 7$ to 2), a residual $\mathrm{pH}$-independent component of the hydrolysis manifests itself. This occurs at a low but constant rate at constant temperature, with the hydrolysis rate varying also depending on the type and concentration of salt (Lowry et al., 1961). Tris buffer, which was utilized in the present study, does not increase the rate of hydrolysis (Anderson and Anderson, 1963). It appears plausible that partial hydrolysis has occurred approximately in the $\mathrm{pH}$-independent regime, affecting the electron density for the nicotinamide. The ribose next to it could then be disordered.

The enzyme appears to be in good density in general and in the coenzyme binding site in particular. The $2^{\prime}$-phosphoadenosine moiety of $\mathrm{NADP}^{+}$also lies in good density, except for the ribose atoms neighboring the diphosphate backbone, which lie in poorer density. The enzyme-coenzyme interactions are chemically reasonable and are by and large similar to those seen in AvIDH and, to a lesser extent, many other more distant IDHs. Results in EcIDH indicated that, when isocitrate was absent, $\mathrm{NADP}^{+}$bound to the coenzyme binding site as a result of the affinity of its adenine moiety (Stoddard et al., 1993). Also, the position of the adenosyl ring of $\mathrm{NADP}^{+}$in the coenzyme binding site was similar when the nicotinamide nucleotide moiety was ordered as when it was disordered (Bolduc et al., 1995; PDB accession code 1IDE). The main determinants of binding of the coenzymes $\mathrm{NADP}^{+}$or NADPH were the adenosyl-ribose and, in $\mathrm{NADP}^{+}$, the $2^{\prime}$-phosphate (Stoddard et al., 1998). Taken together, it appears reasonable to conclude that the observed coenzyme binding interactions in the present crystal 
have not been significantly changed as a result of the presumed partial hydrolysis of nicotinamide and the disorder of the ribose.

\subsubsection{Models for active site access in IDHs}

In EcIDH, the disorder in the nicotinamide nucleotide part of the coenzyme in the absence of the substrate isocitrate (Hurley et al., 1991) was proposed to have another functional significance in an enzyme in which the substrate and coenzyme could bind in random order (Dean and Koshland, 1993): when the coenzyme bound first, the disorder in it appeared to allow isocitrate access to the active site (Stoddard et al., 1993).

A previous kinetics study indicated that a random-order substrate/coenzyme addition model is applicable in the forward direction also in the monomeric AvIDH (Wicken et al., 1972). However, assuming the same applies to $\mathrm{CgIDH}$, disorder in the coenzyme, when it binds first, does not appear to be necessary to allow the substrate access to the active site. This is due to the large hinge movement demonstrated above in CgIDH. Future studies should be able to test the speculation that this may hold for monomeric IDHs in general. In conjunction with the discussion above, the presence of the large hinge movement may have then allowed some monomeric IDHs, such as AvIDH, to evolve into enzymes with a high turnover number while conserving the random order ligand addition model for maximum efficiency. In other monomeric IDHs, such as $\mathrm{CgIDH}$, achieving a higher turnover number appears to continue to be sacrificed on the altar of maintaining a high degree of substrate specificity.

\subsubsection{Apoenzyme in the asymmetric unit}

The concentration of the coenzyme that was used in the crystallization drop (10 $\mathrm{mM}$ ) in the current study was much higher than the $\mathrm{K}_{m}$ of CgIDH towards NADP ${ }^{+}$, which has been determined previously as $4 \mu \mathrm{M}$ (Chen and Yang, 2000) to $24 \mu \mathrm{M}$ (Eikmanns et al., 1995). However, $\mathrm{NADP}^{+}$was found to bind to only one of the two monomeric enzyme molecules in the asymmetric unit in the crystal structure, which indicates crystal packing to be responsible. Two arginines, Arg596 and Arg645, play a key role in binding the $2^{\prime}$-phosphate moiety of $\mathrm{NADP}^{+}$in the holoenzyme. In the Apo form in the crystal, however, Asp506 and Glu508 from a symmetry-related molecule in the crystal come into close proximity to these arginines (Fig 2.12). The former makes an ordered salt bridge and hydrogen bonds with Arg596; the latter is disordered. This interference disrupts coenzyme binding for one of the enzyme molecules in the structure. 


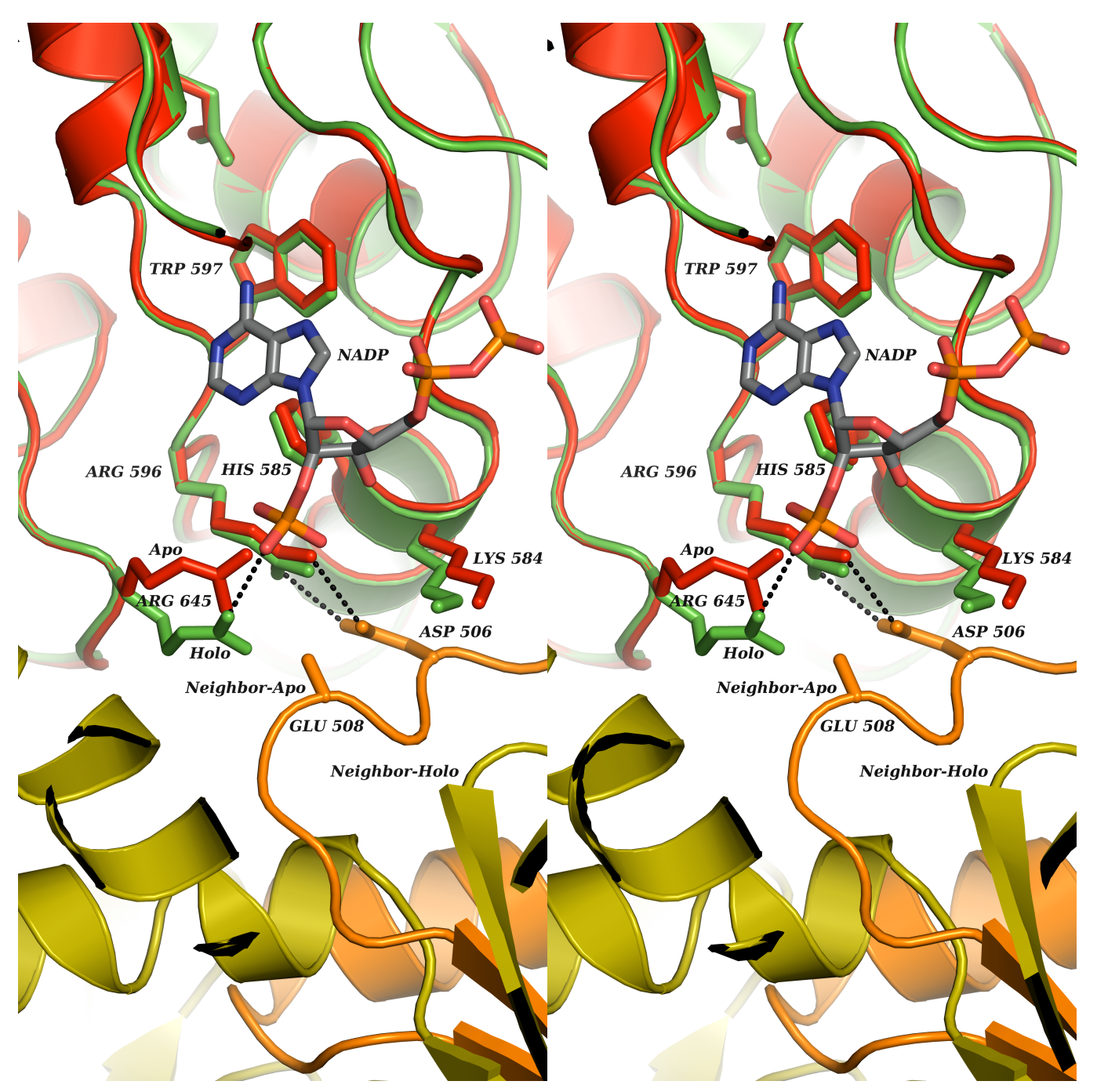

Figure 2.13: Disruption of coenzyme binding in apoenzyme. The Holo (green) and Apo2 (red) forms in the same crystal are overlayed onto each other in the coenzyme binding region. A symmetry-related molecule in the crystal approaches the exposed coenzyme binding site of the Apo 2 form and disrupts coenzyme binding. The symmetry-related molecule of the Holo form is much farther and displays no similar interference with coenzyme binding in that form. Figure published in Sidhu et al. (2011); copyright IUCr; used with permission. 


\subsubsection{Limitations and prospects}

Fully ordered electron density was observed corresponding to only about a third of the $\mathrm{NADP}^{+}$molecule. Although a part of the second third of the molecule appeared relatively well-ordered so that it was modeled, less confidence can be placed in this part of the model. It did not appear to be feasible to model the partial disorder in this distal part of the enzyme based on the residual density seen. It is speculated that $\mathrm{NADP}^{+}$adopts a partially flexible structure in this more distal region. Future experiments should confirm the more precise nature of binding interactions for the central and distal thirds of the coenzyme. The assumed partial hydrolysis of $\mathrm{NADP}^{+}$in this structure should also be addressed in future experiments. This includes through crystallization optimization, which was constrained by the limited availability of the enzyme in the present study. Crystallization and SAXS experiments (the latter as suggested above) with the substrates, other divalent metal ions and inhibitors with the wild-type and mutant enzymes would help to better determine structural determinants of function in this highly specific enzyme.

\subsection{Conclusions}

The highly $\mathrm{NADP}^{+}$-specific monomeric isocitrate dehydrogenase was co-crystallized with $\mathrm{NADP}^{+}$and its crystal structure solved. Specificity-mediating residues in the enzyme that directly interact with the crucial $2^{\prime}$ phosphate of the adenosine ribose in $\mathrm{NADP}^{+}$were identified. Two arginines and one histidine directly bind the negatively charged phosphate by making salt bridge and hydrogen bond interactions with it. In addition, a lysine interacts with the phosphate via water-mediated hydrogen bonds. The enzyme binds the coenzyme in an open conformation in which the coenzyme is highly solvent-exposed but does not block the substrate isocitrate from accessing the active site. Two two-residue hinge points are also indentified in the enzyme. These appear to be involved in conformational changes in the enzyme, as observed for the two molecules in the asymmetric unit in the current structure that differ by a $13^{\circ}$ hinge rotation in the same crystal. It appears significant that one of the hinge point lies in close proximity to the substrate binding site. The structure suggests that isocitrate-binding could involve a conformational change into a more closed conformation of the enzyme. A closed conformation also appears to be the active conformation in this enzyme. It appears plausible that competitive inhibitors of isocitrate could bind to the isocitrate binding site and bring about a conformational change into an inactive form of the enzyme. 



\section{Crystal structure of human sulfamidase}

\subsection{Summary}

Glycosaminoglycans (GAGs; also known as mucopolysaccharides) are linear polysaccharides with a varying degree of $\mathrm{N}$ - and O-sulfations. Their enzymatic degradation occurs in the cell organelles called lysosomes, involving many enzymes. The deficient activity of one of these enzymes leads to accummulation of GAGs and their degradation products in lysosomes, causing rare recessive diseases called mucopolysaccharidoses (MPS).

In the autosomal recessive mucopolysaccharidosis Type III A (MPS IIIA; also known as Sanfilippo A syndrome), the deficient enzyme is sulfamidase, a glycoprotein. The substrates of this enzyme are GAGs heparin and heparan sulfate that have a high negative charge density. The enzyme catalyzes the cleavage of a sulfate attached in an S-N linkage from 2-sulfoglucosamine at the non-reducing terminus of the GAGs. In this disease, typically children 2 to 6 years old present with progressive neurodegeneration that leads ultimately to severe mental retardation and premature death in the second or third decade of life. Milder forms of the disease exist, with delayed onset and slower progression, but have been described less commonly. More than 80 mutations in the sulfamidase gene are associated with this disorder. However, the molecular basis of disease has been poorly understood, a major impediment being the lack of an atomic structure of the enzyme.

In solving the crystal structure of a target molecule using molecular replacement (MR), an attempt is made to place a similarly structured search model in the correct orientation and position in the unit cell (see Chapter 1). Phases calculated for atomic positions of the search model are then combined with experimentally observed diffraction data for the target model to produce what might be described as a "hybrid" electron density map. This map is then interpreted to tease out, if at all possible, the structure of the target molecule. For proteins, MR has often been abandoned if the search model shares a low (typically less than 30\%) sequence identity with the target molecule as the map may be uninterpretable in terms of the target structure. Expanding the threshold for successful MR to search models with lower sequence identity is an area of active research. Here, MR as implemented in the programs ARCIMBOLDO/PHASER was employed to find a rotation-translation solution that was then successfully rebuilt and refined at a low, approximately $22 \%$ sequence identity between the search and target sequences.

Crystal structures of two crystal forms of glycosylated recombinant human sulfamidase were solved. The $2.0 \AA$ and $2.4 \AA$ structures contain between them a total of 10 enzyme monomer chains forming 5 independent homodimers. There are two active sites per dimer. Each monomer contributes one active site, which 
lies in a pocket at the bottom of a surface cleft. It contains a modified cysteine residue in the form of a formylglycine, an approximately octahedrally coordinated $\mathrm{Ca}^{2+}$ ion, and a sulfate binding site.

Despite the low overall sequence identity and significant structural differences between the S-O bond-cleaving sulfatases (hence called O-sulfatases) and the S$\mathrm{N}$ bond-cleaving sulfatase ( $\mathrm{N}$-sulfatase) sulfamidase, the sulfatase fold and 9 of 10 important active site residues are common to both, suggesting divergent evolution and a similar enzyme mechanism, which is proposed. The 10th residue is a lysine in O-sulfatases with known structures but an arginine in human sulfamidase. Models are proposed for how the substrate binds to the enzyme in the active site. Eighty missense mutations associated with disease are mapped onto the 3-dimensional structure of the enzyme, furthering a rational molecular basis to understand impaired enzyme function in this disease. 


\subsection{Introduction}

Glycosaminoglycans (GAGs; also called mucopolysaccharides) are linear polysaccharide chains composed of a repeating disaccharide structural unit with some variable covalent modifications. One of the monosaccharide residues in the disaccharide unit is D-glucosamine or galactosamine; the second tends to be a uronic acid, e.g. D-glucuronic acid or iduronic acid (Jackson et al., 1991). Variable Nand O-sulfations and acetylations of this general scaffold then yield a structurally heterogeneous set of highly negatively charged macromolecules. Major types of GAGs are heparan sulfate, heparin, chondroitin sulfate, dermatan sulfate, keratan sulfate and hyaluronic acid. Many of these GAG types can also occur in a form in which they are covalently attached to a protein: The reducing terminus forms an O-glycosidic bond to a serine or asparagine residue on the protein, forming a proteoglycan. Proteoglycans play diverse structural and functional roles at the cell surface (see Bernfield et al., 1999 for a review) and in the extracellular matrix (reviewed in Iozzo, 1998), including binding various proteins, forming structural components of the basement membrane, and functional roles in cell adhesion and cell signalling.

GAG heparin (5-40 kDa; average approximately $12 \mathrm{kDa})$ is similar in structure to GAG heparan sulfate $(5-50 \mathrm{kDa}$; average approximately $30 \mathrm{kDa})$ but the former tends to be much more heavily sulfated than the latter. Indeed, heparin is believed to possess the highest negative charge density amongst biological macromolecules (for reviews, see Capila and Linhardt, 2002; Linhardt, 2003). Heparin is also used medically as an anticoagulant. GAG heparan is thought to be present on most cell surfaces and appears to be targeted by at least some pathogens to gain entry into cells, e.g. by the dengue virus (Chen et al., 1997b).

In humans, the degradation of GAGs is catalyzed by lysosomal enzymes. The deficient activity of one of these enzymes can lead to an accummulation of the substrate GAGs and their alternative degradation products in the lysosome, resulting in lysosomal storage disorders called mucopolysaccharidoses (MPS). As of now, 11 different MPS disorders stemming from deficient activities of different enzymes have been well described (for reviews, see Neufeld and Muenzer, 2001; Giugliani, 2012).

A block in the degradation of heparan sulfate and heparin leads to clinical conditions called mucopolysaccharidoses III (MPS III; also known as Sanfilippo syndrome) in which CNS signs and symptoms predominate (for a review, see Valstar et al., 2008). Other organs are comparatively less affected, especially initially. In the autosomal recessive MPS III Type A (MPS IIIA; Sanfilippo A syndrome; MIM 252900) (Sanfilippo et al., 1963), the activity of the lysosomal enzyme sulfamidase (also known as N-sulfoglucosamine sulfohydrolase or SGSH, sulfamate sulfohydrolase and heparan N-sulfatase; EC 3.10.1.1; MIM 605270) is deficient. 


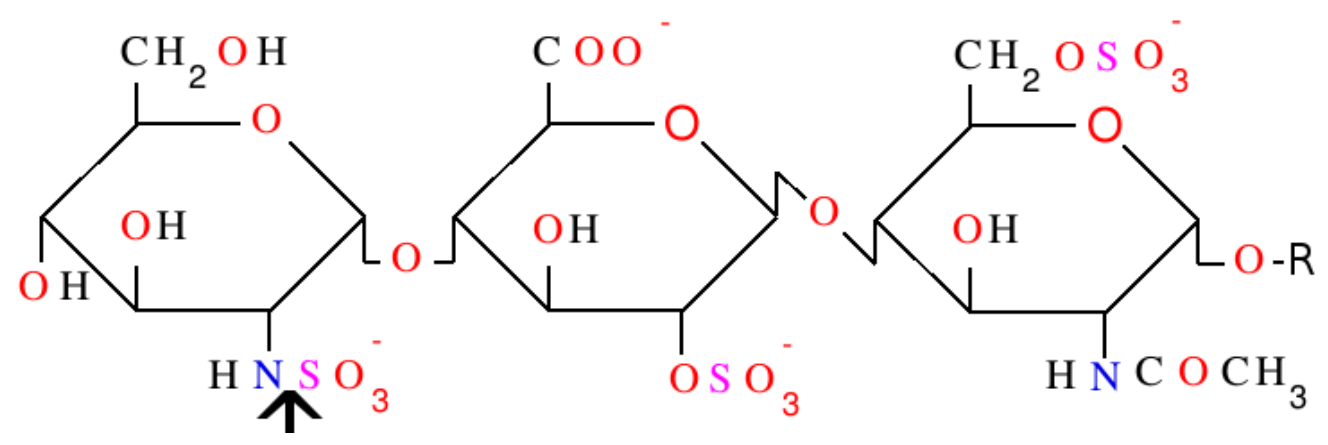

Figure 3.1: A representative schematic structure of heparan sulfate. The enzyme catalyzes the cleavage of the $\mathrm{S}-\mathrm{N}$ bond from the 2-sulfoglucosamine residue at the non-reducing terminus of the polysaccharidic glycosaminoglycans heparin and heparan sulfate. The location in the structure of the bond cleaved is indicated by an upward-pointing arrow. $\mathrm{R}$ (extreme right) represents the rest of the substrate chain.

MPS IIIA typically manifests itself in children at the age of 2 to 6 years. Initial neurological signs and symptoms are mild and can easily be missed. CNS degeneration worsens over time, ultimately leading to severe mental retardation. Premature death often occurs in the second or third decade of life. Less commonly, milder forms of the disease have been described that occur with a later onset and slower progression. MPS IIIA is currently incurable; therapy is supportive.

Over 80 mutations in the sulfamidase gene that are associated with the disease have been described. However, the molecular basis of how the mutations affect enzyme function is poorly understood. One of the major hurdles has hitherto been the lack of a 3-dimensional structure of the human, indeed any, sulfamidase. Overcoming this hurdle is the major focus of this thesis.

Sulfamidase is a glycoprotein that catalyzes the hydrolytic cleavage of Nlinked sulfate groups attached to the terminal 2-glucosamine residue at the nonreducing end of heparin and heparan sulfate (Fig. 3.1). The products of the reaction are the GAG with a desulfated glucosamine residue at the non-reducing end, and a free sulfate ion.

Sulfamidase belongs to the sulfatase family of enzymes (for reviews, see Hanson et al. 2004; Ghosh, 2007). A common feature of the arylsulfatase subfamily of sulfatases is a sulfatase fold with a highly conserved CXPSR sequence motif in the active site, where $X$ is a serine or a threonine residue. The cysteine in this motif is post-translationally modified to a formylglycine (FGly) residue that plays a crucial role in catalysis. This modification takes place enzymatically, in a reaction catalyzed by the FGly-generating enzyme (Dierks et al., 2005). In contrast with $\mathrm{O}$-sulfatases, which catalyze the cleavage of an S-O bond in O-linked sulfates, 
sulfamidase is an N-sulfatase that acts on an S-N bonded sulfate. Sulfamidase appears to be one of only two hydrolases EC-recognized as cleaving an S-N bond, the other one being cyclamate sulfohydrolase (EC 3.10.1.2). No crystal structure is available for either of these two $\mathrm{N}$-sulfatases. In addition, the enzyme shares a low sequence identity with some of the O-sulfatases for which atomic structures have been determined. Thus our understanding of the enzymatic mechanism and the molecular basis of various mutations is relatively poor.

One of the common methods used to solve crystal structures is molecular replacement (MR). In this technique, part or whole of a molecule with a known structure (search model) that is similar to the target structure that we desire to solve is used as a starting point. X-ray diffraction data obtained on a crystal of the target molecule are used to attempt to orient and position the search model in the crystal similarly to the target structure (rotation-translation solution). Phases derived from this preliminary solution are used to calculate an electron density map, which is interpreted to tease out the structure of the target model, if possible. It is an iterative approach of structure building and refinement of model parameters against the diffraction data. The success of the technique depends crucially on the degree of similarity of the search model to the target molecule. For proteins, one of the major determinants of similarity is the level of amino acid sequence identity of the search and target molecules. A 35 to $30 \%$ sequence identity has been the borderline zone below which the MR technique has often been abandoned as the electon density map becomes uninterpretable in terms of the target structure and cannot be improved (see Chapter 1).

As part of this thesis, the 3-dimensional structure of glycosylated recombinant human sulfamidase was solved for two different crystal forms of the enzyme. The search model used shared a low sequence identity of approximately $22 \%$ with sulfamidase. The electron density map was practically uninterpretable initially. The model was refined against data to a resolution of $2.00 \AA$ and $2.40 \AA$, respectively, for the two crystal forms. It is the first structure of a sulfamidase from any species and apparently also the first structure of a hydrolase recognized to cleave a sulfate bonded in an S-N bond.

\subsection{Materials and Methods}

\subsubsection{The sulfamidase gene}

The $11 \mathrm{~kb}$ sulfamidase gene was previously mapped to chromosome 17 locus 17q25.3, the gene cloned and the nucleotide sequence determined (Genbank accession number U30894) (Scott et al., 1995). It consists of 8 exons (Karageorgos et al., 1996). The 1506-nucleotide open reading frame coded for a 502 amino-acid protein. The first 20 amino acids were predicted to be the signal peptide that is 


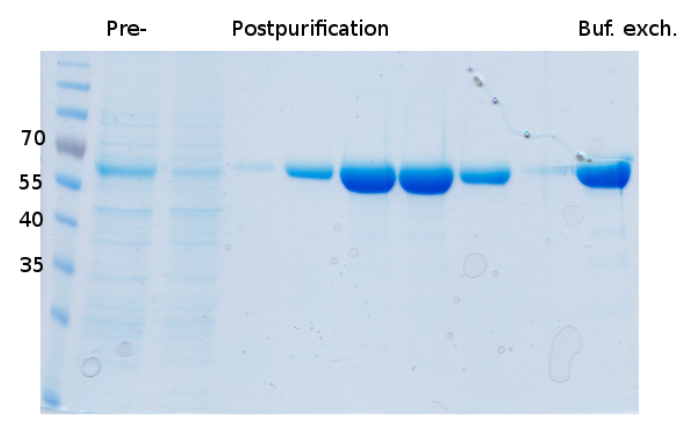

Figure 3.2: Sulfamidase purity. SDS-PAGE of the enzyme before (columns 2 and 3 from left) and after purification (columns 4-9) and buffer exchange (column 10) steps (reference molecular weight standards in column 1). Figure first published in Sidhu et al. (2014); copyright IUCr; used with permission.

cleaved by a peptidase to give a 482 amino acid mature protein with a calculated molar mass of $54.7 \mathrm{kDa}$.

\subsubsection{Protein expression and purification}

Purified SGSH was obtained from the laboratory of Prof. Dr. Robert Steinfeld, Dept. of Neuropediatrics, University of Goettingen. In brief, to help with purification, a short linker followed by a 6-His tag (RSHHHHHH) was engineered to be expressed at the C-terminus of SGSH. HEK 293 cells were transfected with the SGSH cDNA construct and successfully transfected cells selected for as described previously (Steinfeld et al., 2004). The cell culture medium was centrifuged at $3000 \mathrm{~g}$ and $4{ }^{\circ} \mathrm{C}$ for 60 minutes. The supernatant was filtered through a membrane with a pore size of $0.2 \mu \mathrm{m}$ and the solution loaded onto a HisTrap HP column (GE Healthcare) in $20 \mathrm{mM} \mathrm{K}_{2} \mathrm{HPO}_{4}(\mathrm{pH} 7.5), 0.5 \mathrm{M} \mathrm{NaCl}$ and 40 $\mathrm{mM}$ imidazol. A step gradient of imidazol was used to release bound SGSH. The enzyme eluted at [imidazol] $\geq 100 \mathrm{mM}$.

\subsubsection{Protein crystallization trials}

Purified SGSH was placed on ice and the enzyme purity and integrity confirmed in collaboration with the Steinfeld laboratory using sodium dodecylsulfate polyacrylamide gel electrophoresis (SDS-PAGE) on aliquots from before and after the purification and buffer exchange steps, confirming the successful purification of a band of approximately $65 \mathrm{kDa}$, consistent with the approximate molecular weight of a subunit of the over-expressed protein (Fig. 3.2).

Pure fractions were pooled together. The enzyme was then buffer-exchanged 
into a solution of $10 \mathrm{mM}$ Tris $\cdot \mathrm{HCl}(\mathrm{pH} 7.5), 100 \mathrm{mM} \mathrm{NaCl}$, and concentrated to approximately $10 \mathrm{mg} / \mathrm{mL}$ using centrifugation through a filter (Millipore). This protein solution was stored on ice at $4{ }^{\circ} \mathrm{C}$ and used for crystallization trials.

Two different diffraction-quality crystal forms of SGSH were obtained: a small cell (S) form, and a large cell (L) one. The former was obtained during robotic crystallization-condition screening experiments, and the latter during manual optimization, both using the sitting-drop vapor-diffusion method at 293 $\mathrm{K}$.

The crystal form $\mathrm{S}$ was obtained in 96-well Greiner low-profile plates. A well volume of $100 \mu \mathrm{L}$ was pipetted for each of the different conditions using the robot TECAN Genesis RSP 150. A sitting-drop volume of $0.1 \mu \mathrm{L}$ was then set up by mixing 1:1 the well and protein solutions using the robot TTP Labtech Mosquito. Plate- and rod-shaped crystals of maximum dimensions approximately $20 \mu \mathrm{m} \mathrm{x}$ $20 \mu \mathrm{m} \times 50 \mu \mathrm{m}$ were obtained over several weeks, with optimal conditions being $25 \%$ (w/v) polyethylene glycol (PEG) 3350, $200 \mathrm{mM} \mathrm{MgCl}_{2}$ and $100 \mathrm{mM}$ HEPES buffer ( $\mathrm{pH} 7.5)$.

For crystal form L, rod-shaped diffraction-quality crystals with typical dimensions of approximately $50 \mu \mathrm{m} \times 50 \mu \mathrm{m} \times 350 \mu \mathrm{m}$ were obtained in $13 \%(\mathrm{w} / \mathrm{v})$ PEG $8000,200 \mathrm{mM} \mathrm{MgCl}_{2}$ and $100 \mathrm{mM}$ Bis-Tris buffer (pH 5.1). The crystals were cryoprotected using a solution of approximately 20 to 25\% (w/v) PEG 8000 with otherwise conserved composition and flash-frozen in liquid $\mathrm{N}_{2}$.

\subsubsection{Data collection}

For both crystal forms, native single-crystal X-ray diffraction datasets were collected at the Swiss Light Source beamline PXII (X10SA) using monochromatic radiation of wavelength $0.99989 \AA$ An a Dectris Pilatus 6M detector with a crystalto-detector distance of $400 \mathrm{~mm}$. The oscillation range was $0.1^{\circ}$ and the exposure time $0.1 \mathrm{~s}$. A total of 3,600 and 1,800 images were collected for crystal forms $\mathrm{S}$ (Fig. 3.3) and L, respectively. Data integration was performed using the program XDS (Kabsch, 2010). Crystal form $\mathrm{S}$ was found to be split but integration was successfully performed by using a larger integration box size.

The space group was determined to be $\mathrm{P} 2{ }_{1}$ for both the large and small cells. Unit cell parameters for crystal form $\mathrm{S}$ were $\mathrm{a}=61.4 \AA \mathrm{A}, \mathrm{b}=107.9 \AA \mathrm{A}, \mathrm{c}=79.8 \AA$ and $\beta=104.1^{\circ}$. Crystal form $L$ had a 4.5 times larger cell volume than the $S$ form, with cell parameters $\mathrm{a}=103.0 \AA, \mathrm{b}=211.6 \AA, \mathrm{c}=108.4 \AA$ and $\beta=102.7^{\circ}$. Correlation coefficient $\mathrm{CC}_{1 / 2}$ of the data (Karplus and Diederichs, 2012) was used to determine data truncation threshold. Its value for the $\mathrm{S}$ form was approximately $64 \%$ at a resolution limit of $2.00 \AA$, and $65 \%$ at $2.40 \AA$ for the $L$ form, both values being determined as implemented in the program XPREP (Sheldrick, 2012), which was also used for further data reduction. Data collection and processing 


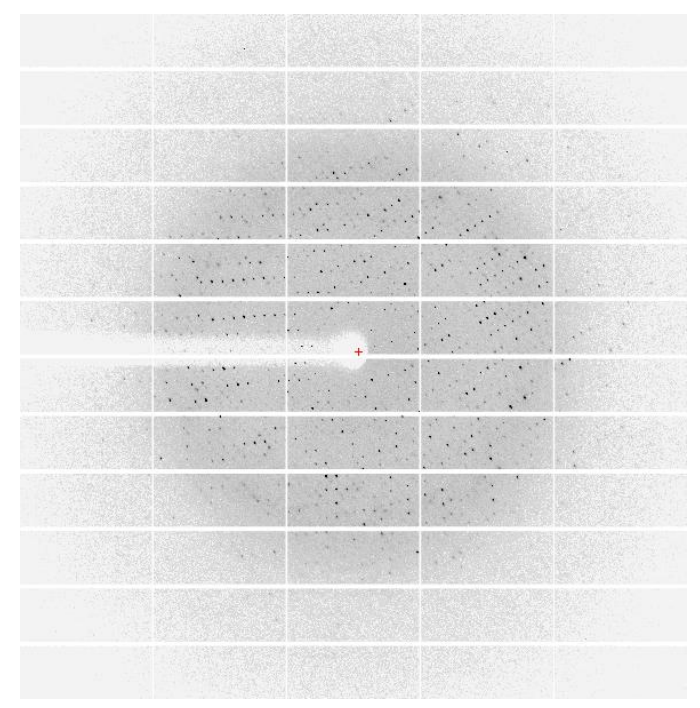

Figure 3.3: High-resolution diffraction from a sulfamidase crystal (crystal form S).

statistics are listed in Table 3.1 (Results section below).

\subsubsection{Structure refinement}

All data for crystal form $S$ were used for the structure solution step (described below). For cross validation in refinement, $5 \%$ of the data were set aside randomly as the Rfree set for the small cell; for the large cell, $8462(5 \%)$ reflections in 21 thin shells ( 2.45 to $48.9 \AA$ ) were set aside using the program SFTOOLS (Collaborative Computational Project, Number 4 [CCP4], 1994; Winn et al. 2011). The resolution range was later extended to $2.40 \AA$; conservation of the $\mathrm{R}_{\text {free }}$ set was achieved using MTZ2HKL (Grune, 2008) and XPREP (Sheldrick, 2012).

Model building was done using the program COOT (Emsley et al., 2010), and maximum-likelihood refinement of model coordinates and B factors against the working set data using REFMAC 5.5 (Murshudov et al., 1997, 2011). Water molecules were added either automatically or manually. Waters were in general manually deleted if evidence for them in the map was weak [refined $2 m F_{o}-D F_{c}$ density (Read, 1986) showing up at a contour level less than $1.0 \sigma$, or if the shape of the density was too non-spherical], if the refined $B$ factor values were too high (typically over $80 \AA^{2}$ ), if the waters were in general closer than $2.3 \AA$ from neighboring atoms or more distant than $3.5 \AA$ from protein atoms. Towards the end of refinement, hydrogen atoms were added to the structure in riding positions. TLS parameters could be successfully refined when TLS domains were defined based on visual inspection of the structure. The final $\mathrm{R}_{\text {free }} / \mathrm{R}_{\text {work }}$ (Brünger, 1992) 
was $22.99 \% / 19.83 \%$ for crystal form S and $24.02 \% / 20.89 \%$ for crystal form L. RMSD's for superpositions of the SGSH monomer were calculated using INDONESIA (Madsen et al., 2002), and those for the dimer using LSQKAB (Kabsch, 1976).

Accessible surface area for each residue in chain A in crystal form S was calculated using the Protein Interfaces, Surfaces and Assemblies (PISA) server (Krissinel and Henrick, 2007) at the European Bioinformatics Institute, http://www.ebi.ac.uk/pdbe/prot_int/pistart.html. For ease of comparison across different types of amino acid residues, the accessible surface area was converted into normalized accessible surface area (NASA) as follows:

$$
N A S A / \%=100 \frac{A_{\text {res }} / n_{\text {res }}}{A_{\text {Lys } 490} / n_{\text {Lys } 490}},
$$

where $A_{\text {res }}$ is the accessible surface area of a given residue, $n_{\text {res }}$ is the number of atoms in the residue (calculated using an in-house program; NSS, unpublished), $A_{L y s 490}$ is the accessible surface area of Lys490 (the highly surface-exposed internal residue with the maximum value of accessible surface area per atom per residue in the chain, specifically $26.9 \AA^{2}$ per atom per residue), and $n_{\text {Lys } 490}$ is the number of atoms in Lys490. Dimer interface interactions were evaluated visually and using the PISA server. Figures were rendered using PYMOL (DeLano, 2002) and an in-house program (NSS, unpublished: Fig. 3.8).

Multiple sequence alignment was performed by first running a search for amino acid sequences homologous to the sequence of human sulfamidase using the BLASTP server of the National Library of Medicine using default parameters. Closest sequences tentatively annotated as those of sulfamidases were blindly retrieved from the results, followed by their sequence alignment using the CLUSTAL OMEGA server (Goujon et al., 2010).

\subsection{Results}

\subsubsection{Buffer exchange and crystallization}

The mature human sulfamidase consists of 482 amino acids (residues 21 through 502 in the amino acid sequence), with a calculated molar mass of $55 \mathrm{kDa}$ for the non-glycosylated monomer. The purified glycosylated enzyme was obtained from the laboratory of Prof. Robert Steinfeld and buffer exchanged in preparation for crystallization. Crystallization trials were conducted upon confirmation of purity and integrity of the enzyme before and after buffer exchange (Methods section).

In crystallization trials, two different crystal forms of sulfamidase were obtained (Fig. 3.4a-d). They are denoted as S (for small unit cell) and L (large unit cell). The $\mathrm{S}$ form crystallized at a $\mathrm{pH}$ of 7.5; the $\mathrm{L}$ form at a $\mathrm{pH}$ of 5.1. 

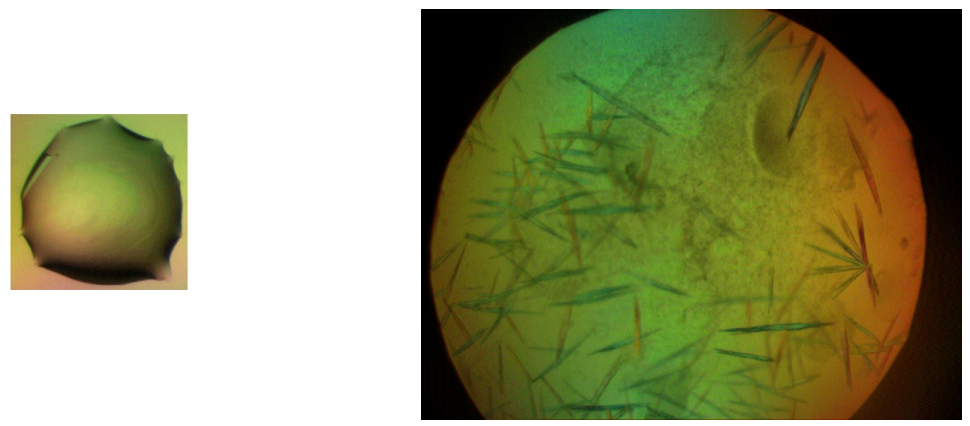

(a) Crystal form $\mathrm{S}$.

(b) Needles of crystal form L

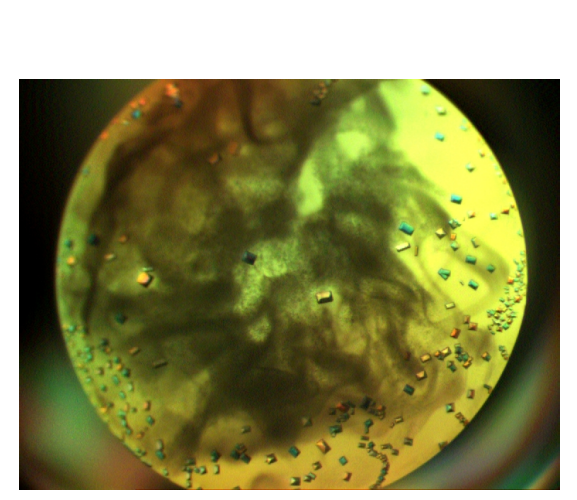

(c) Showers of crystal form L.

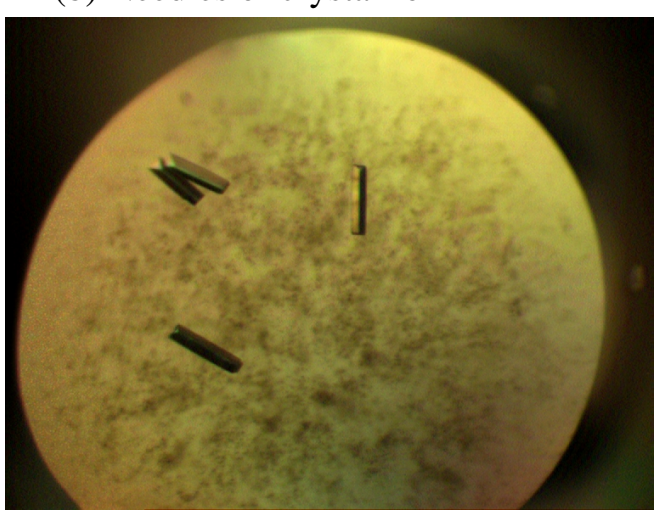

(d) Crystals of L form.

Figure 3.4: Sulfamidase crystallization and crystal optimization trials. Crystals of crystal forms S (panel a) and L (b-d). Typical crystal dimensions upon optimization were approximately $20 \mu \mathrm{m} \times 20 \mu \mathrm{m}$ x $50 \mu \mathrm{m}$ for the $\mathrm{S}$ form and up to $50 \mu \mathrm{m}$ x $50 \mu \mathrm{m}$ x $350 \mu \mathrm{m}$ for the $\mathrm{L}$ form. 


\subsubsection{Structure solution of crystal form $S$}

Nineteen different sulfuric-ester hydrolases (EC 3.1.6.1 through 3.1.6.19; O-sulfatases) are recognized (ExplorEnz enzyme database of the Enzyme Nomenclature List, International Union of Biochemistry and Molecular Biology, http://www.enzyme-database.org/, accessed July 17, 2013). Structures have been described for 5 of these (Research Collaboratory for Structural Bioinformatics [RCSB] Protein Data Bank [PDB], http://www.rcsb.org/pdb/, accessed July 17, 2013):

1) arysulfatase A (ASA; also known as human lysosomal cerebroside-3-sulfate 3-sulfohydrolase; EC 3.1.6.8; PDB IDs 1N2K, 1N2L, 1E33, 1E1Z, 1E3C, 1E2S, 1AUK),

2) arylsulfatase B (ASB; human lysosomal N-acetylgalactosamine-4-sulfate 4-sulfohydrolase; N-acetylgalactosamine-4-sulfatase; EC 3.1.6.12; PDB ID 1FSU),

3) arylsulfatase from Pseudomonas aeruginosa (PAS; EC 3.1.6.1; PDB ID 1HDH),

4) human estrone/dehydroepiandrosterone sulfatase (ES; steryl-sulfatase; EC 3.1.6.2; PDB ID 1P49), and

5) human lysosomal (N-acetyl)galactosamine-6-sulfatase (GALNS; N-acetylgalactosamine-6-sulfatase EC 3.1.6.4; PDB ID 4FDI, 4FDJ).

Of these, the $1.3 \AA$ crystal structure model of a bacterial sulfatase from $\mathrm{Pseu}$ domonas aeruginosa (Boltes et al., 2001; PDB ID 1HDH) was found to possess a structure-based sequence identity of approximately $22 \%$ with the sequence of human sulfamidase. This model was selected for an attempt at molecular replacement (MR) using PHASER (McCoy et al., 2007) as implemented in the program ARCIMBOLDO (Rodríguez et al., 2009, 2012). X-ray diffraction data for the sulfamidase crystal form $\mathrm{S}$ was chosen for this attempt as it diffracted to a higher resolution of $2.0 \AA$, which has been seen to be important for these attempts. Due to the poor sequence identity, structure-based sequence alignments were performed also with other O-sulfatase homologues to identify possible conserved secondary structure elements.

The search model was used as a source of search model fragments for MR using ARCIMBOLDO/PHASER run on a computer grid by Prof. Isabel Usón. A rotation-translation (R-T) solution with figures of merit that made it distinctly stand out over other possible solutions was obtained, suggesting that it could be a potentially true solution. The translation $\mathrm{Z}$ score for this R-T solution was 7.5. There were 2 molecules in the asymmetric unit.

However, several aspects of the R-T solution promised difficulties ahead. 1

\footnotetext{
${ }^{1}$ Especially in case of a poor search model, it is only after a significant degree of success in model rebuilding and refinement that more useful structural features of the target molecule begin
} 
The starting $\mathrm{R}_{\text {free }}$ had essentially random values of approximately $53.3 \%$. Density modification and chain tracing by SHELXE (Sheldrick, 2002, 2008; Thorn and Sheldrick, 2013) was unsuccessful in this case. Structures with $\beta$-sheet cores have been seen to be more difficult to solve using this approach than those with an $\alpha$-helix core. Consistent with this, initial refinement attempts failed. The electron density was highly model-biased (as the phases were derived from the search model rather than the true structure) and essentially uninterpretable.

Fig. 3.5 illustrates the quality of the electron density at this stage, along with a model of the rotation-translation solution. The final SGSH model is also shown for comparison. Electron density is highly misleading. It has a strong tendency to follow the rotation-translation model rather than the final model, which was unknown at this stage. Remodeling is crucial, but electron density is the only "direction pointer" and it is difficult to distinguish where it is pointing in the correct direction and where in a misleading one. A significant bettering of the electron density demands a significant remodeling of the structure into a generally correct direction, which is time-consuming and can in general be difficult or even impossible to arrive at. The rotation-translation solution is a correct one but it can be a challenging task to advance from it.

Fig. 3.6 shows the density in another region, illustrating the translational and (correlated) rotational displacement of the long helix, the true position of which often lies outside the density. The density corresponding to a loop (lower center) also points to the wrong position. There is also density corresponding to a helix (bottom of figure) that is non-existent in the true structure. In a few places, the density points to structure building in directions that correspond to neither the rotation-translation model nor the true structure. In Fig. 3.7 is illustrated a region in which at some positions the electron density indicates the true direction of chain tracing, followed by a wrong direction.

Systematic attempts were made to improve the position and orientation of the R-T solution: Parts of the model that appeared to be more likely to be variable between sulfatases and sulfamidase were removed from the solution. Side-chains were removed to prevent them from trapping the model in local minima. Lowresolution rigid body refinement trials were performed in PHASER, starting with a single rigid body and slowly increasing the number along with the resolution. At each major step, low-resolution rigid body refinement tests were conducted using REFMAC. Indicators monitored included the log likelihood gain, model fit to density, possible signs of improvement in interpretable electron density, figure of merit (REFMAC), and $\mathrm{R}_{\text {free }} / \mathrm{R}_{\text {work }}$. After some trial and error, a model with improved figures of merit was obtained. However, the model was now highly

to show up in the electron density. Otherwise the rotation-translation solution has to be abandoned and the structure is unsolved. 


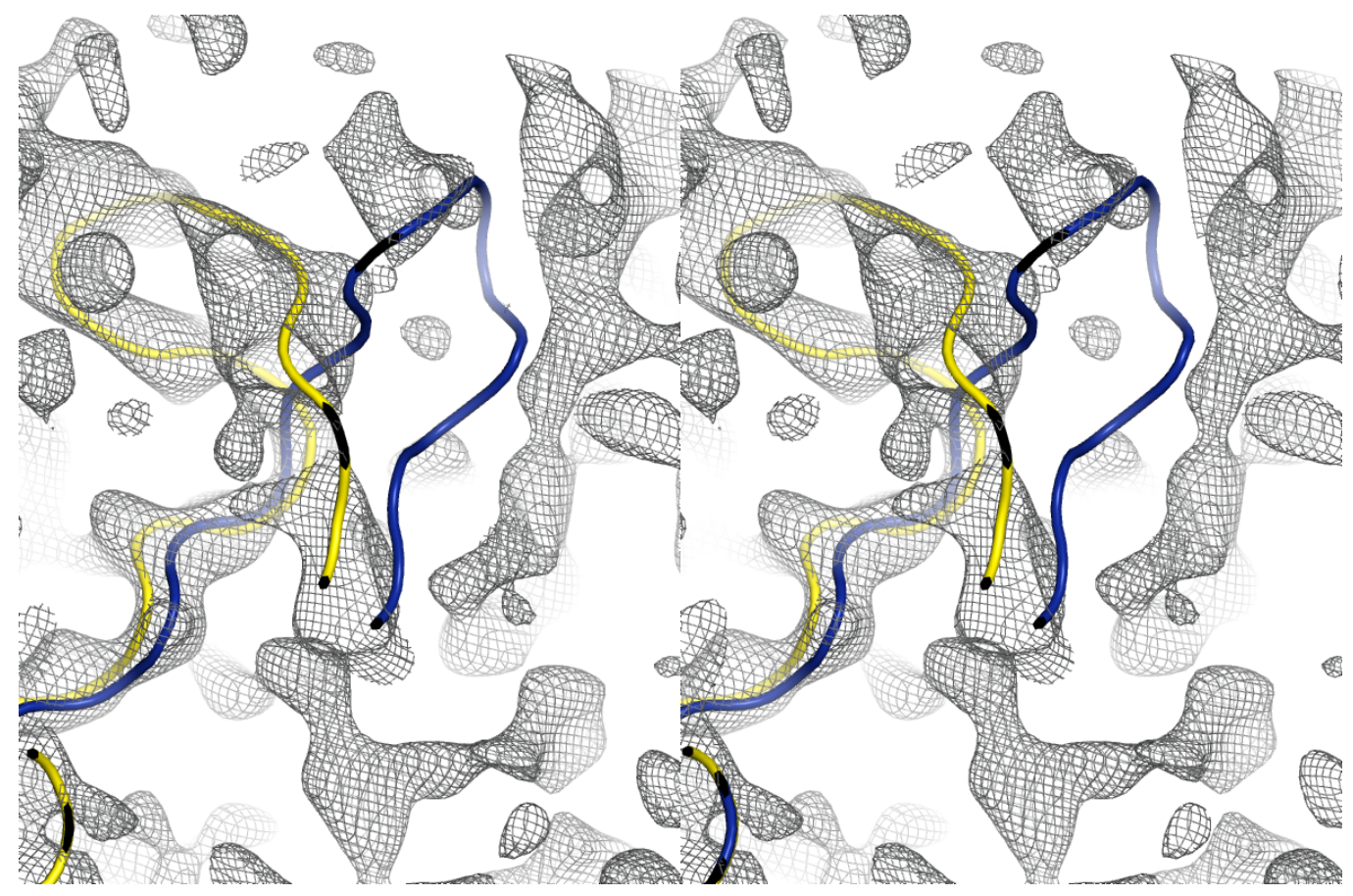

Figure 3.5: Model bias at the rotation-translation solution stage. Stereo view of the rotation-translation solution model (yellow) and the corresponding electron density (gray) at this stage. The final model of SGSH (blue), which was unknown at this point is also shown (blue) for comparison. The $2 m F o-D F c$ electron density map (Read, 1986) is contoured at the $1.0 \sigma$ level. 


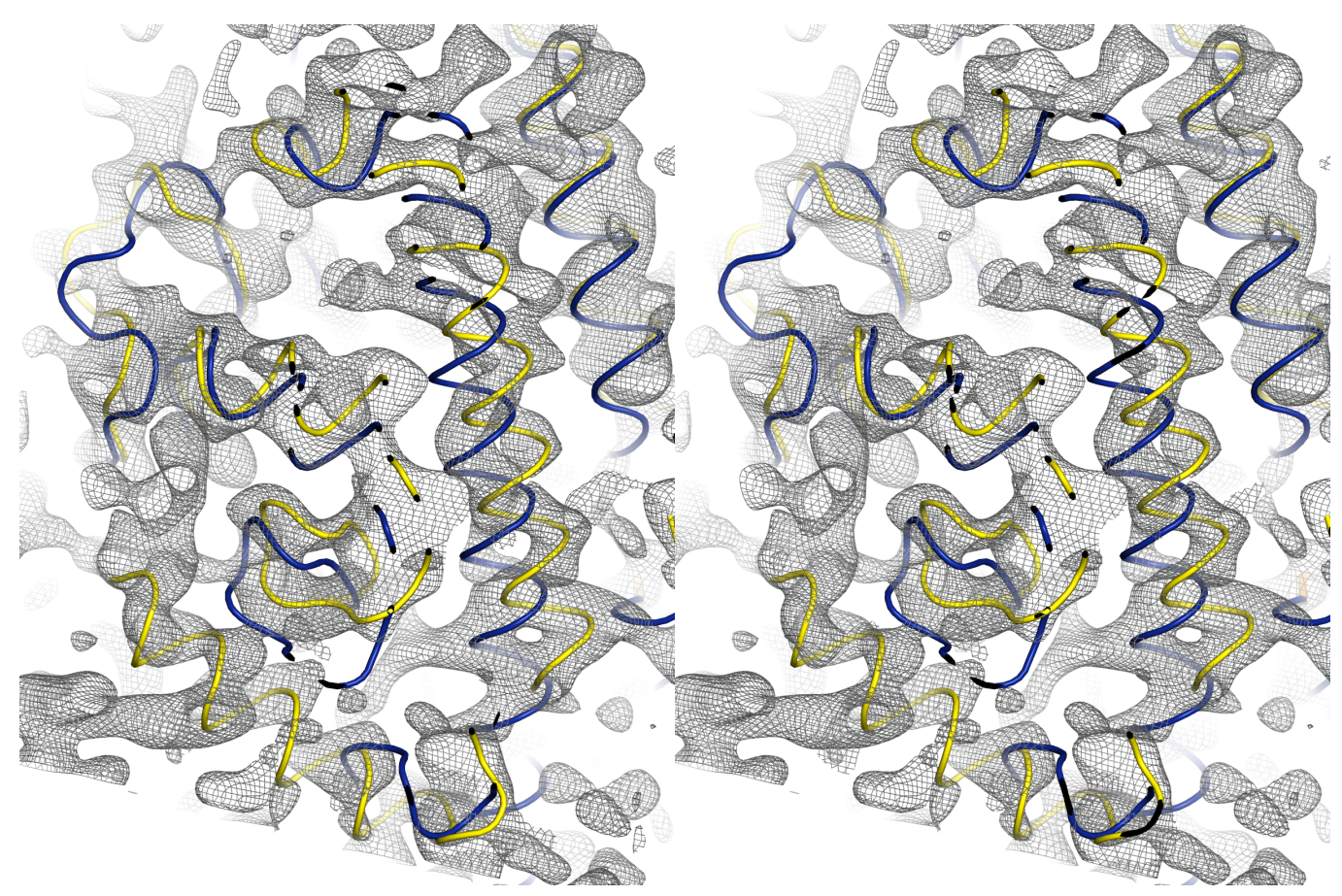

Figure 3.6: Stereo view of model bias in another region of the map. The long helix is at the right, a misplaced loop in the lower center, a helix that is non-existent in the true structure at the bottom. Representation as in Fig. 3.5, with the rotation translation solution in yellow, the electron density contoured at the same level in gray, and the as yet unknown final sulfamidase model in blue. 


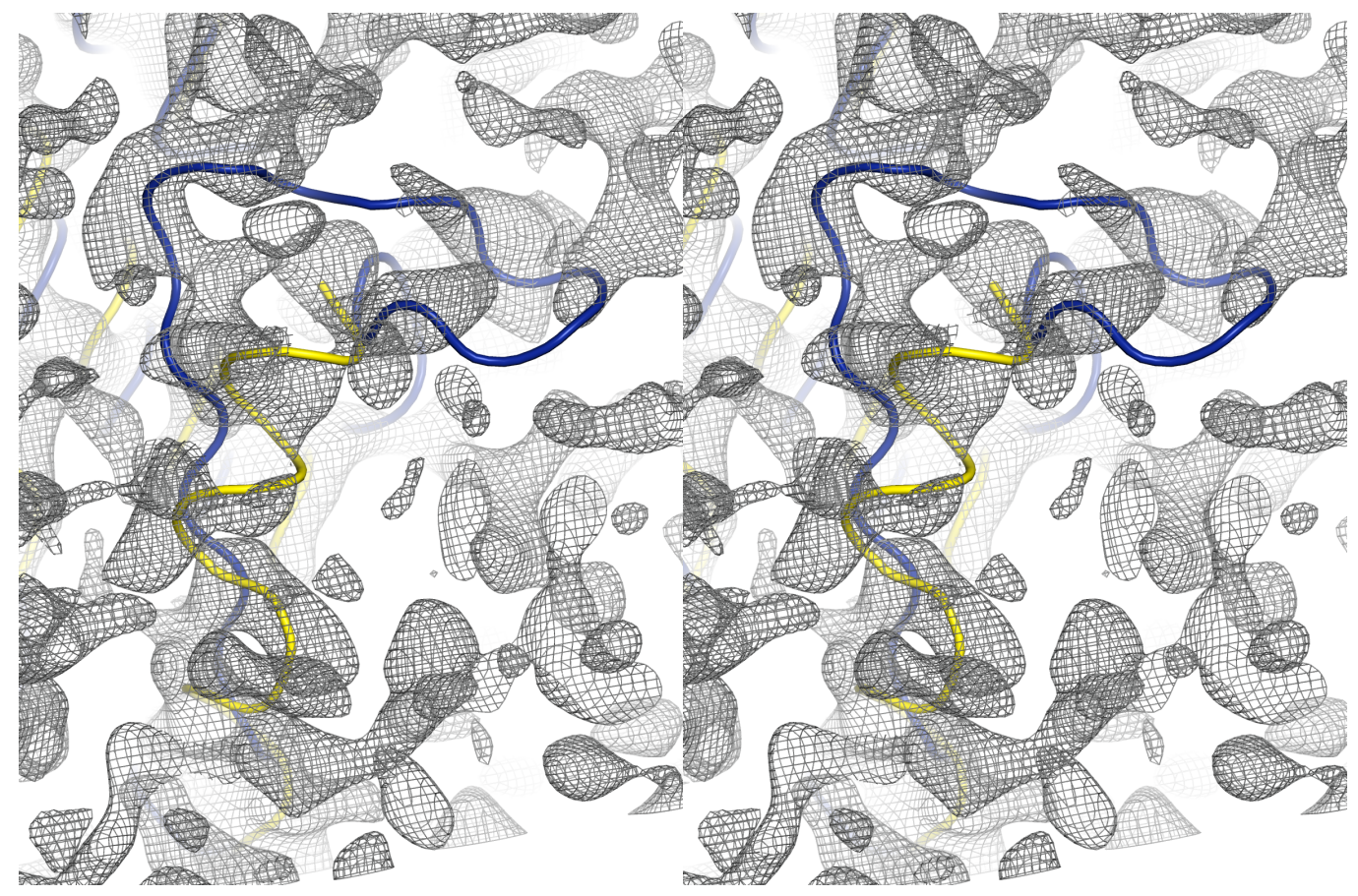

Figure 3.7: Stereo view of electron density pointing in biased, correct and noisy directions. Density apparently suggestive of a correct direction to model in (towards the top, where it overlaps with the blue but not the yellow model) then points in a direction that appears to be noise. Representation as in Fig. 3.5 and 3.6. 
incomplete and the density still appeared to be uninterpretable. Due to the poor nature of the electron density map, it was unclear where to rebuild the main chain or what side chain rotamers to model. The program SCWRL4 (Krivov et al., 2009) was used to model secondary structure-based (pruned) side chain rotamers. A combination of the above approaches resulted in the first signs of a (possibly) correct solution: in just a few places in the map, the difference density seemed to show small buds of positive difference density, suggesting that visual rebuilding might be commenced. However, possibly due to the degree of model incompleteness, progress was painstakingly slow initially, albeit over time it became more significant and cautiously promising.

\subsubsection{Refinement of form $S$ and structure solution of form $L$}

At this stage, the significantly incomplete model was used as an MR search model to solve the crystal structure of crystal form L using PHASER. Eight molecules could be located in the asymmetric unit of this crystal form. Due to the existence now of 10 molecules in 2 different crystal forms a successful attempt was made at density modification with electron density averaging over the 10 molecules in the 2 crystals. This was accomplished using the CCP4 (Collaborative Computational Project, Number 4, 1994) program DM-MULTI (Cowtan, 1994). This procedure helped to obtain a significantly improved electron density map for crystal form $\mathrm{S}$ (the main focus of refinement due to its higher resolution). A modest but significant improvement could now be made in the speed of structure building.

Manual modeling could later be combined with piecemeal corrections of errors in the model being built using a combination of density modification with DM-MULTI and automatic chain tracing by the CCP4 program BUCCANEER (Cowtan, 2006). Upon obtaining a relatively complete structure model for crystal form $\mathrm{S}$, it was used as an improved search model to obtain a better structure solution for crystal form L. Structure building and refinement was then completed for both crystal forms S and L, and the structure published (Sidhu et al., 2014).

\subsubsection{Model quality}

The crystal form $\mathrm{S}$ contains 2 molecules per asymmetric unit and was refined against data up to $2.00 \AA$. The $\mathrm{L}$ form has 8 molecules per asymmetric unit and was refined to $2.40 \AA$. The enzyme appears as a homodimer in both crystal forms, 1 in crystal form $\mathrm{S}, 4$ in crystal form $\mathrm{L}$.

For crystal form $\mathrm{S}$, continuous electron density was observed of a quality approximately similar for both molecules (chain A and B) in the asymmetric unit. For crystal form L, the quality was significantly better for half of the molecules (chains A-D) as compared to the rest (chains E-H). 
However, a structure model of approximately similar coverage (typically 480 residues per chain, residues 22 through 501 in sequence) could be built for all 10 chains in the two crystal forms. Additional residues could be built into some chains (e.g. in crystal form $\mathrm{L}$, residue 21 in chain $\mathrm{C}$, and residues 502 through 505 in chain A). A few internal residues (185-186) are missing in the model due to poor density in chain $\mathrm{F}$, crystal form $\mathrm{L}$.

Protein $\phi / \psi$ angles were not restrained in refinement and so these could be used as an indicator of model quality. For crystal form S, the Ramachandran plot calculated using the MOLPROBITY server shows 97.1\% (933/961) of amino acid residues in the favored, 99.8\% (959) in the allowed, and $0.21 \%$ (2) in the outlier region (Ramakrishnan and Ramachandran, 1965; Lovell et al., 2003). For crystal form $\mathrm{L}$, the equivalent values were similar to the ones above: $97.2 \%(3727 / 3835)$, $99.7 \%$ (3823) and $0.31 \%$ (12), respectively.

Despite the different conditions in the two crystal forms, Asp94 is an outlier in all 10 chains. It lies in good density. It is the only outlier in the higher resolution crystal form $\mathrm{S}$. These observations suggest that it is a true outlier rather than an error.

In the lower resolution form L, there are 4 additional outliers in 3 of 8 chains: Lys339 in chains C, D and F, and Asp168 in chain F. Of these, Lys339 is a loop residue that lies in reasonable density in these 3 chains. Asp168 is also a loop residue that lies in density of weak quality in chain $\mathrm{F}$.

Data collection and refinement statistics are presented in Table 3.1.

Chain A in crystal form $\mathrm{S}$ will be described by default for the monomer; chains $\mathrm{A}$ and $\mathrm{B}$ in the same crystal form for the dimer. Other chains will be specified where appropriate.

\subsubsection{Sulfamidase monomer}

The enzyme monomer has a globular structure, with overall dimensions approximately $40 \AA \times 50 \AA \times 70 \AA$. It consists of two domains, a larger N-terminal domain (Domain 1) and a smaller C-terminal domain (Domain 2). There is no obvious hinge point between the two domains that could serve as a domain boundary. Approximately, Domain 1 can be thought to consist of residues 21 through 356 and Domain 2 of residues 357 through 502. The active site resides in Domain 1. Overall, the monomer belongs to the sulfatase fold.

Both domains have a $\beta$-sheet at their centers, with $\alpha$ helices wrapped around them. Overall, the monomer contains $14 \beta$ strands ( $\beta 1$ through $\beta 14$ ), $13 \alpha$ helices ( $\alpha 1$ through $\alpha 13$ ) and six 310 helices (T1-T6) (Fig. 3.8) [classification as per Kabsch and Sander (1983), as implemented in the CCP4 program PROCHECK].

Domain 1 is of the $\alpha / \beta$ form, with a central core made up of a mixed $\beta$ sheet of $8 \beta$ strands, as indicated in a topology diagram (Fig. 3.9) and a corresponding 
Table 3.1: Data collection and refinement statistics

\begin{tabular}{|c|c|c|}
\hline Crystal form (PDB ID) & S (4MHX) & L (4MIV) \\
\hline Space group & $\mathrm{P} 21$ & $\mathrm{P} 21_{1}$ \\
\hline \multicolumn{3}{|l|}{ Unit cell parameters } \\
\hline $\mathrm{a}, \mathrm{b}, \mathrm{c}(\AA)$ & $61.4,107.9,79.8$ & $103.0,211.6,108.4$ \\
\hline$\beta\left(^{\circ}\right)$ & 104.1 & 102.7 \\
\hline \multicolumn{3}{|l|}{ Data Collection } \\
\hline Wavelength $(\AA)$ & 0.99989 & 0.99989 \\
\hline Resolution range $(\AA)$ & $44.3-2.00(2.10-2.00)$ & $48.9-2.40(2.50-2.40)$ \\
\hline No. of reflections measured & 452472 & 599199 \\
\hline No. of unique reflections & 67940 & 174779 \\
\hline Redundancy & $6.65(6.38)$ & $3.41(3.45)$ \\
\hline $\mathrm{R}_{\text {merge }}$ & $0.0886(0.6539)$ & $0.0702(0.4723)$ \\
\hline $\mathrm{R}_{\text {sigma }}$ & $0.0500(0.5504)$ & $0.0594(0.4917)$ \\
\hline Completeness (\%) & $99.9(99.8)$ & $99.3(98.7)$ \\
\hline Mean $I / \sigma(I)$ & $11.20(1.85)$ & $11.03(2.08)$ \\
\hline \multicolumn{3}{|l|}{ Refinement } \\
\hline Resolution limit ( $\mathrm{A})$ & $44.3-2.00$ & $48.85-2.40$ \\
\hline $\mathrm{R}_{\text {free }}$ (no. of reflections) & $0.2299(3447)$ & $0.2447(8462)$ \\
\hline $\mathrm{R}_{\text {work }}$ & 0.1920 & 0.2157 \\
\hline Total no. of reflections (working set) & 64437 & 166273 \\
\hline Solvent content $(\%)$ & 45.9 & 53.0 \\
\hline No. of molecules in the asu & 2 & 8 \\
\hline No. of non-H atoms refined & 7968 & 31122 \\
\hline No. of water molecules refined & 205 & 475 \\
\hline Mean B factor, protein atoms $\left(\AA^{2}\right)$ & 44.7 & 64.5 \\
\hline Mean B factor, backbone atoms $\left(\AA^{2}\right)$ & 43.5 & 64.2 \\
\hline Mean B factor, side-chain atoms $\left(\AA^{2}\right)$ & 45.9 & 64.8 \\
\hline Mean $B$ factor, water atoms $\left(\AA^{2}\right)$ & 39.0 & 37.6 \\
\hline Mean $B$ factor, A chain $\left(\AA^{2}\right)$ & 45.5 & 48.9 \\
\hline Mean $B$ factor, B chain $\left(\AA^{2}\right)$ & 43.9 & 44.1 \\
\hline Mean $B$ factor, $C$ chain $\left(\AA^{2}\right)$ & & 48.3 \\
\hline Mean $B$ factor, D chain $\left(\AA^{2}\right)$ & & 44.9 \\
\hline Mean B factor, E chain $\left(\AA^{2}\right)$ & & 85.5 \\
\hline Mean B factor, $F$ chain $\left(\AA^{2}\right)$ & & 75.8 \\
\hline Mean $B$ factor, $G$ chain $\left(\AA^{2}\right)$ & & 79.6 \\
\hline Mean B factor, H chain $\left(\AA^{2}\right)$ & & 93.0 \\
\hline \multicolumn{3}{|l|}{ RMSDs from ideal geometry } \\
\hline Bond length $(\AA)$ & 0.0125 & 0.0028 \\
\hline Bond angles $\left({ }^{\circ}\right)$ & 1.564 & 0.683 \\
\hline \multicolumn{3}{|l|}{ Ramachandran statistics } \\
\hline Favored region $(\%)$ & $97.1(933 / 961)$ & $97.2(3727 / 3835)$ \\
\hline Allowed region $(\%)$ & $99.8(959 / 961)$ & $99.7(3823 / 3835)$ \\
\hline Outlier region (\%) (number of residues) & $0.21(2 / 961)$ & $0.31(12 / 3835)$ \\
\hline
\end{tabular}

Values in parentheses are for the outermost resolution shell. Number of atoms is the occupancy sum; B factors are occupancy-weighted means. B factor values for individual chains refer to protein atoms in the chain. Table adapted from Sidhu et al. (2014). 


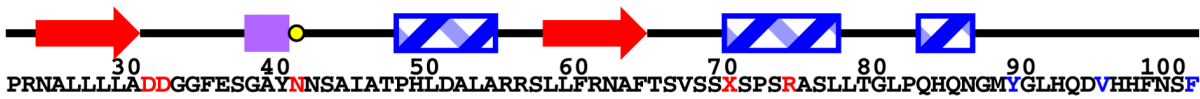
N SN $\mathrm{S}$

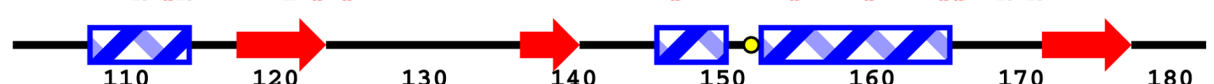
DKVRSLPLLLSQAGVRTGIIGKKHVGPETVYPFDFAYTEENGSVLQVGRNITRIKLIVRKFLQTQDDRPFFLYVAFHDPH

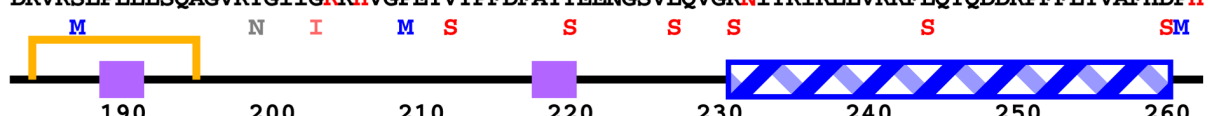

182 RCGHSQPQYYGTFCEKFGNGESGMGRIPDWTPQAYDPLDVLVPYFVPNTPAARADLAAQYTTTVGRMDQGVGLVLQELRDAG $\begin{array}{lllllllll}I & S N & M & S & S S & N & S N & M\end{array}$

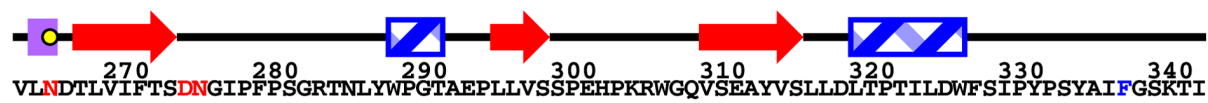
VLNDTLVIFTSDNGIPFPSGRTNLYWFGTAEPLLVSSPEHPKRWGQVISEAYVSLLDLTPTILDWFSIPYPSYAIFGSKTI $\mathrm{S}$

342

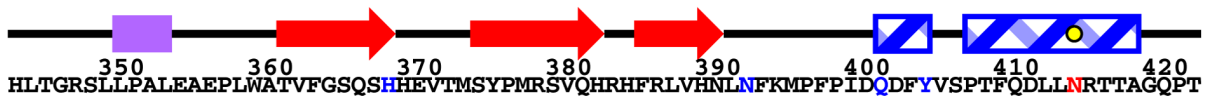

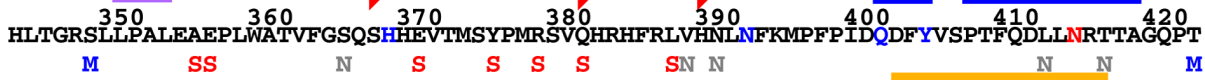

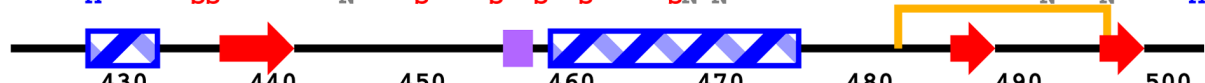

422

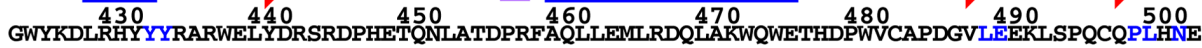
$\mathrm{S}$ M S

N $\mathrm{S}$

LRSH

Figure 3.8: Sequence mapped to secondary structure elements. $\alpha$ helices (blueoutlined boxes with zigzag lines), $\beta$ strands (red arrows), $3_{10}$ helices (violet boxes), disulfide bridges (orange lines), glycosylation sites (yellow circles). Mutations are marked severe (red, labeled S), intermediate (orange, I), mild (blue, M) and of a phenotype not reported (gray, N). 
three-dimensional figure (Fig. 3.10a-c). All except one of the strands in the sheet are parallel. Nine decorating $\alpha$ helices wind around the sheet. One of these (helix $\alpha 7$ ) is 30 residues long. Domain 2 consists mainly of a 4 -stranded antiparallel $\beta$ sheet core surrounded by $4 \alpha$ helices. In addition, it has a C-terminal extension consisting of a small two-stranded anti-parallel $\beta$-sheet.

There are two intra-monomer disulfide bridges, one in each domain, and no free cysteines. The first disulfide bridge (Cys183-Cys194) stabilizes a long mainly looped segment $\beta 5-\alpha 7$. The second (Cys481-Cys495) ties the terminal strand $\beta 14$ to the more proximal loop $\alpha 13-\beta 13$. Electron density corresponding to four glycosylated asparagines was observed next to Asn41, Asn151, Asn264 and Asn413. Four cis peptide bonds were modeled, between Gly127, Asp179, Ala482 and Ser492 and the prolines following each of these residues. All of these occur in good electron density.

To assess the flexibility of the 10 monomer chains, their backbone $\mathrm{C} \alpha$ atoms were superimposed on each other and $\mathrm{C} \alpha$ r.m.s.d.'s calculated. The r.m.s.d. for the 2 chains in crystal form $S$ is $0.20 \AA$; for the 8 chains in the $L$ form, it ranges from 0.10 to $0.17 \AA$. The values are expected to be low, however, as refinement was done with local NCS restraints included: their exclusion was tested for but led to a worsening of $\mathrm{R}_{\text {free }}$. 


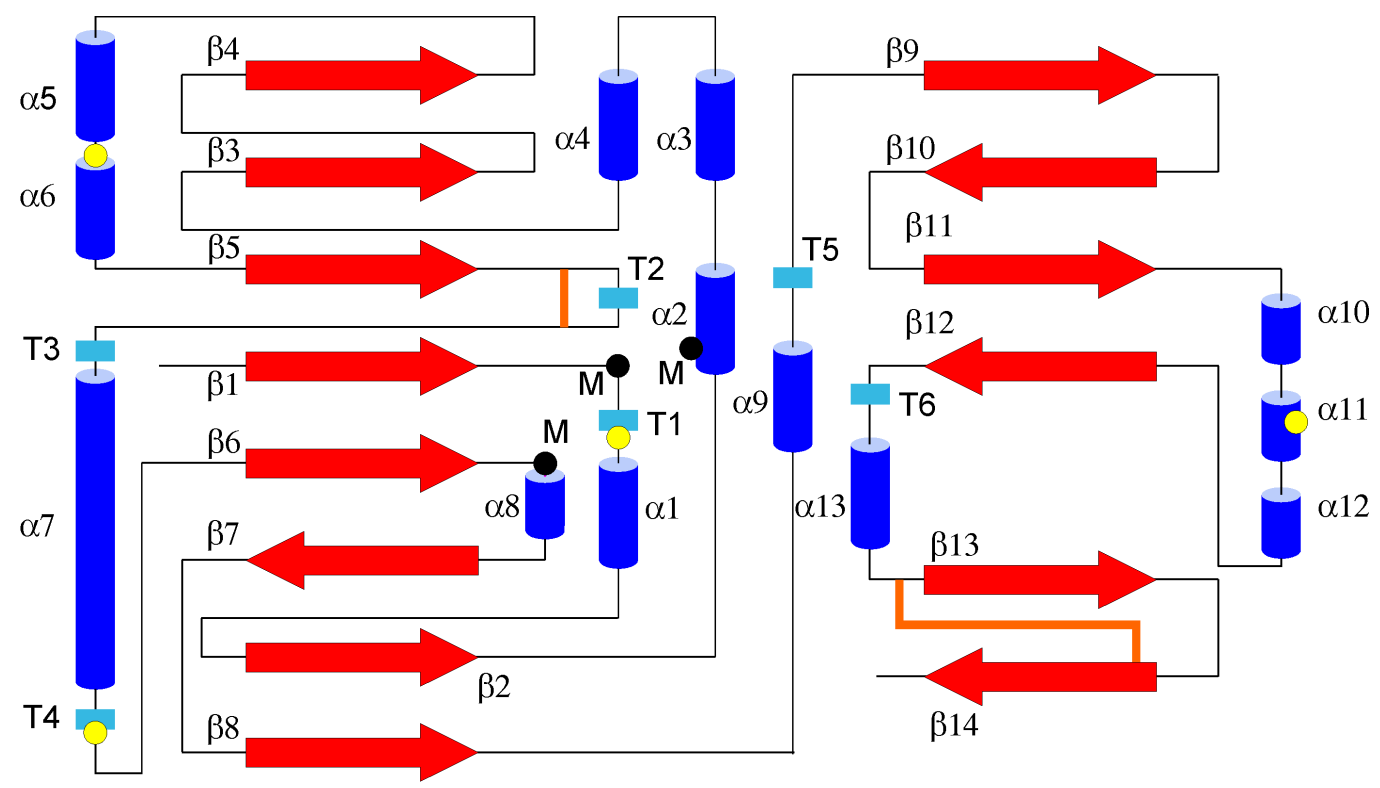

Figure 3.9: Topology diagram of SGSH. Domain 1 consists of the beta sheet on the left, with its associated helices; Domain 2 is on the right. Color coding is similar to that in Fig. 3.8, except for alpha helices, which are shown here as blue cylinders. The divalent metal-binding residues lie at approximate positions in the secondary structure labeled ("M"). Different secondary structure elements are labeled according to their order in the sequence; $33_{10}$ helices (T1-T6). Figure published in Sidhu et al. (2014); copyright IUCr; used with permission. 

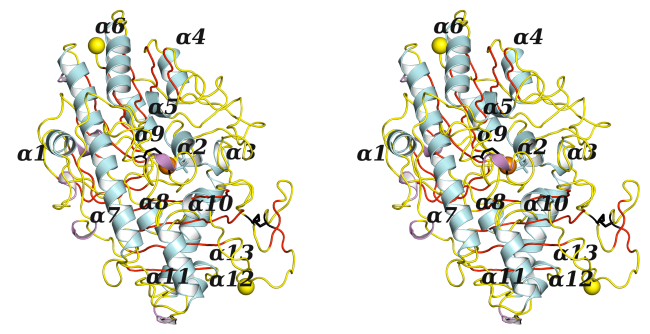

(a) $\alpha$-helices (blue)
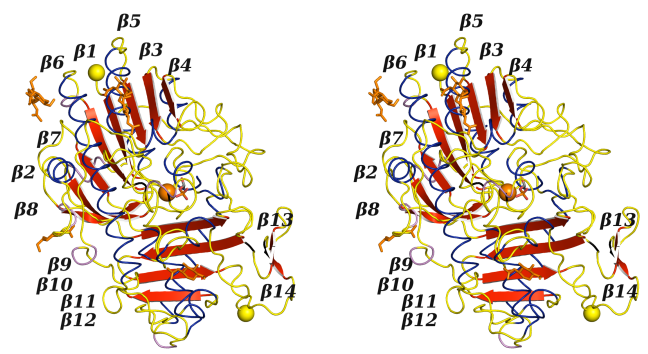

(b) $\beta$-strands (red)
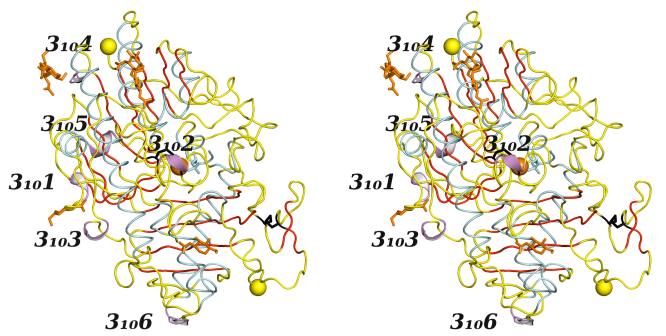

(c) $3_{10}$ helices (T1-T6; violet)

Figure 3.10: Stereo view of secondary structure elements in a subunit (front view). Shown are also: $\mathrm{N}$ and $\mathrm{C}$ terminus (yellow balls at top and bottom, resp.) $\mathrm{Ca}^{2+}$ (orange ball). 


\subsubsection{The homodimer}

Two monomers of the enzyme come together within the asymmetric unit to form a homodimer. The subunits lie $40.8 \AA$ apart from each other (centroid-centroid distance) in the dimer. Approximately $10.3 \%\left(1,900 \AA^{2}\right)$ of the accessible surface area of the monomer $\left(18,400 \AA^{2}\right.$; calculated using PISA, CCP4) is buried in the dimer interface; approximately $11.6 \%$ (56) of the residues participate in forming the dimer interface (calculated using PISA as part of CCP4).

In Fig. 3.11 through 3.14, the secondary structure elements in the homodimer are shown from four different aspects. The first of these aspects (Fig. 3.11) will be referred to as the "front" view in the following sections. In the front view, the noncrystallographic 2-fold axis relating the two subunits passes lies in the plane of the paper (with subunit centroids approximately in the plane on either side). The NCS 2-fold axis passes close to approximately 5 residues that therefore lie close to equivalent residues from the other subunit: Phe98, His367, Ser373, Asn391, Lys393 and Met394.

The dimer interface is stabilized by at least two salt bridges in a solventexposed region of the interface: His185-Glu488' (where the prime indicates a residue from the other subunit in the dimer) and His185'-Glu488. The interface is further stabilized by 11 residues that participate in significant hydrogen bonding interactions: Tyr89, Va195, Lys393, Met394, Tyr403, Arg428, Tyr431, Tyr432, Pro497, Leu498 and Asn500. Other interactions at the interface include polar (e.g. between the pair His499-Thr416), $\pi$-stacking (e.g. Phe101-Phe336), van der Waals, and water-mediated interactions.

Fig. 3.15 displays the asymmetric unit of crystal form L consisting of 8 monomers that form 4 dimers. No evident compact higher-order oligomeric structure was observed in this crystal form. To assess the flexibility of the dimer interface, all 5 homodimer molecules in the two crystal forms were superimposed on each other based on one of the subunits in each dimer. The relative orientations of the non-superimposed subunits differ within a range of 2.6 to $3.0^{\circ}$.

\subsubsection{Active site}

Fig. 3.16 indicates the location of the active site in relation to the enzyme surface and the dimer interface. There are two active sites in the homodimer. Each of them lies in a narrow pocket at the bottom of a surface cleft (Fig. 3.17). The entrance to the active site is markedly lined with positively charged residues (represented as blue balls). Each monomer wholly contributes one active site. However, the part of the second subunit lies close to the entry to the active site pocket and partly contributes to the cleft in which the pocket lies. The active site contains a catalytically crucial formylglycine 70 (FGly70), a divalent metal-binding site and 

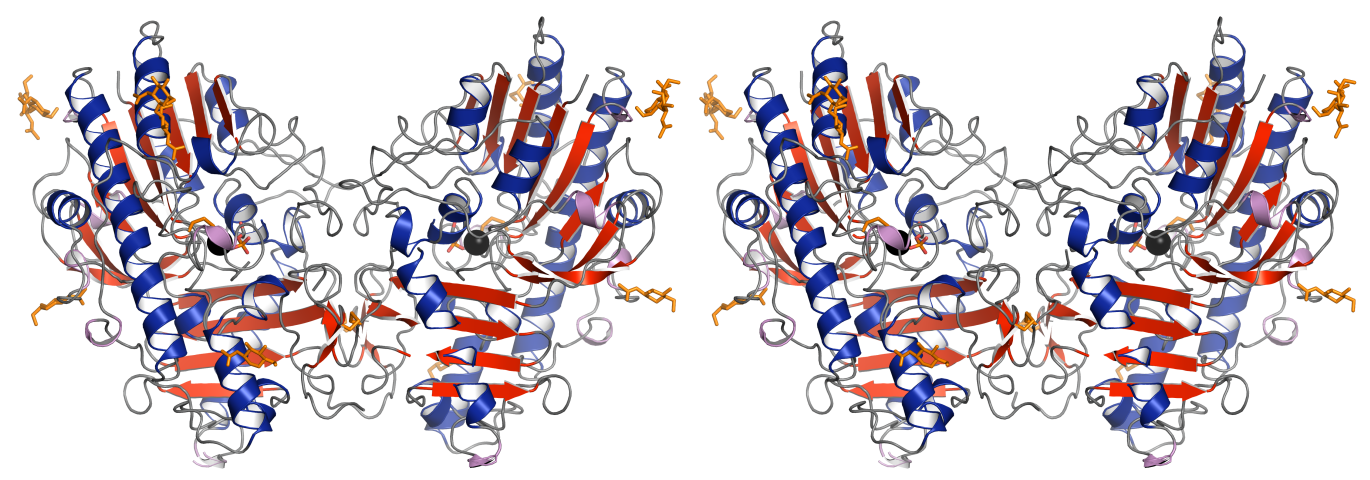

Figure 3.11: Stereo front view of sulfamidase dimer. The NCS 2-fold relating the two monomers lies vertically in the plane of the paper; subunit centroids also lie (approximately) in the paper plane on either side of the NCS 2-fold. Coloring is by secondary structure elements ( $\alpha$ helices in blue, $\beta$ sheets in red, $3_{10}$ helices in violet and loops in gray). The active site FGly70, cystin disulfide bridges and glycosylations are shown as orange stick models. The active site $\mathrm{Ca}^{2+}$ is shown as a black ball. The rest of the views are described as top, bottom and side (opposite the second dimer subunit) with respect to this view. Figure published in Sidhu et al. (2014); copyright IUCr; used with permission. 

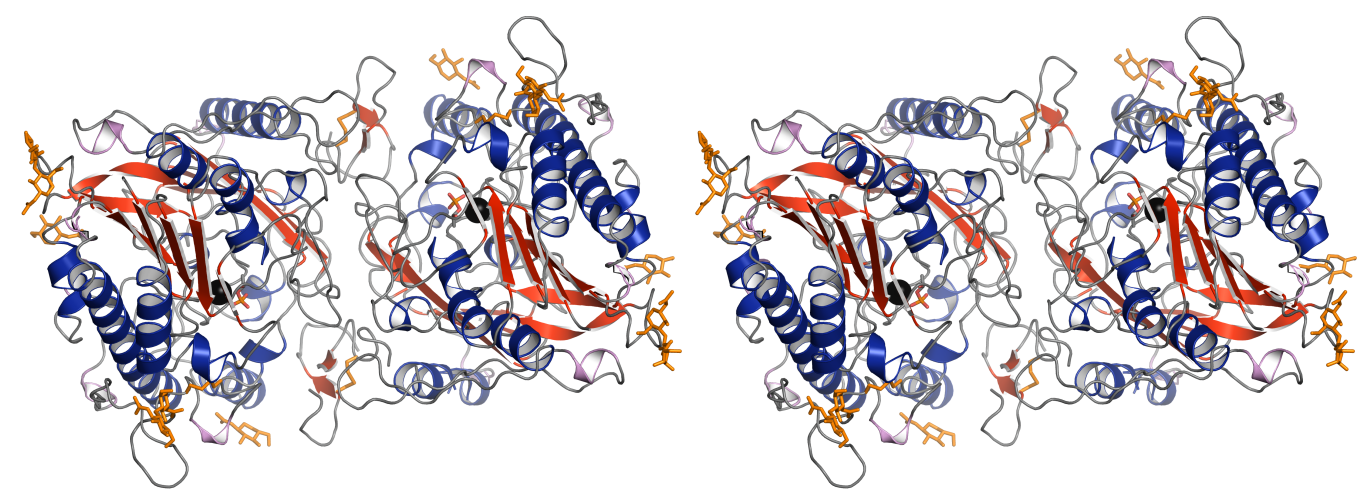

Figure 3.12: SGSH dimer, top view in stereo. Representation as in Fig. 3.11.
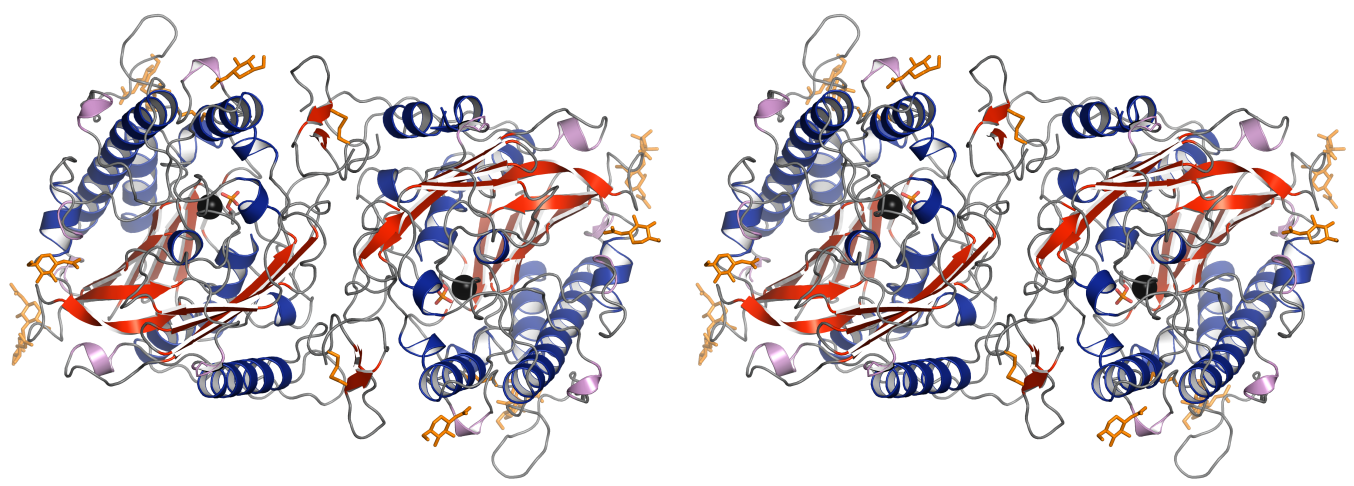

Figure 3.13: SGSH dimer, bottom view in stereo. Representation as in Fig. 3.11. 

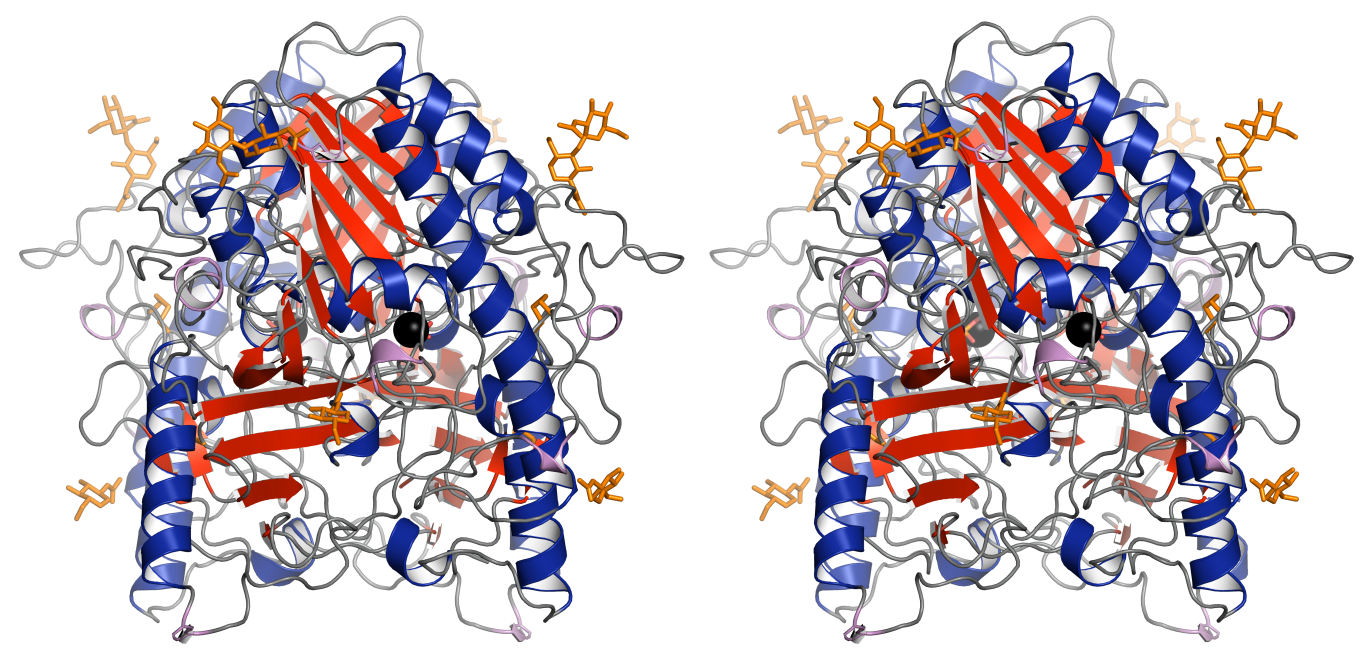

Figure 3.14: SGSH dimer, side view (stereo). This orientation was achieved from that in the front view by a 90-degree rotation around the vertical axis anticlockwise when looking from the top. Representation as in Fig. 3.11. 


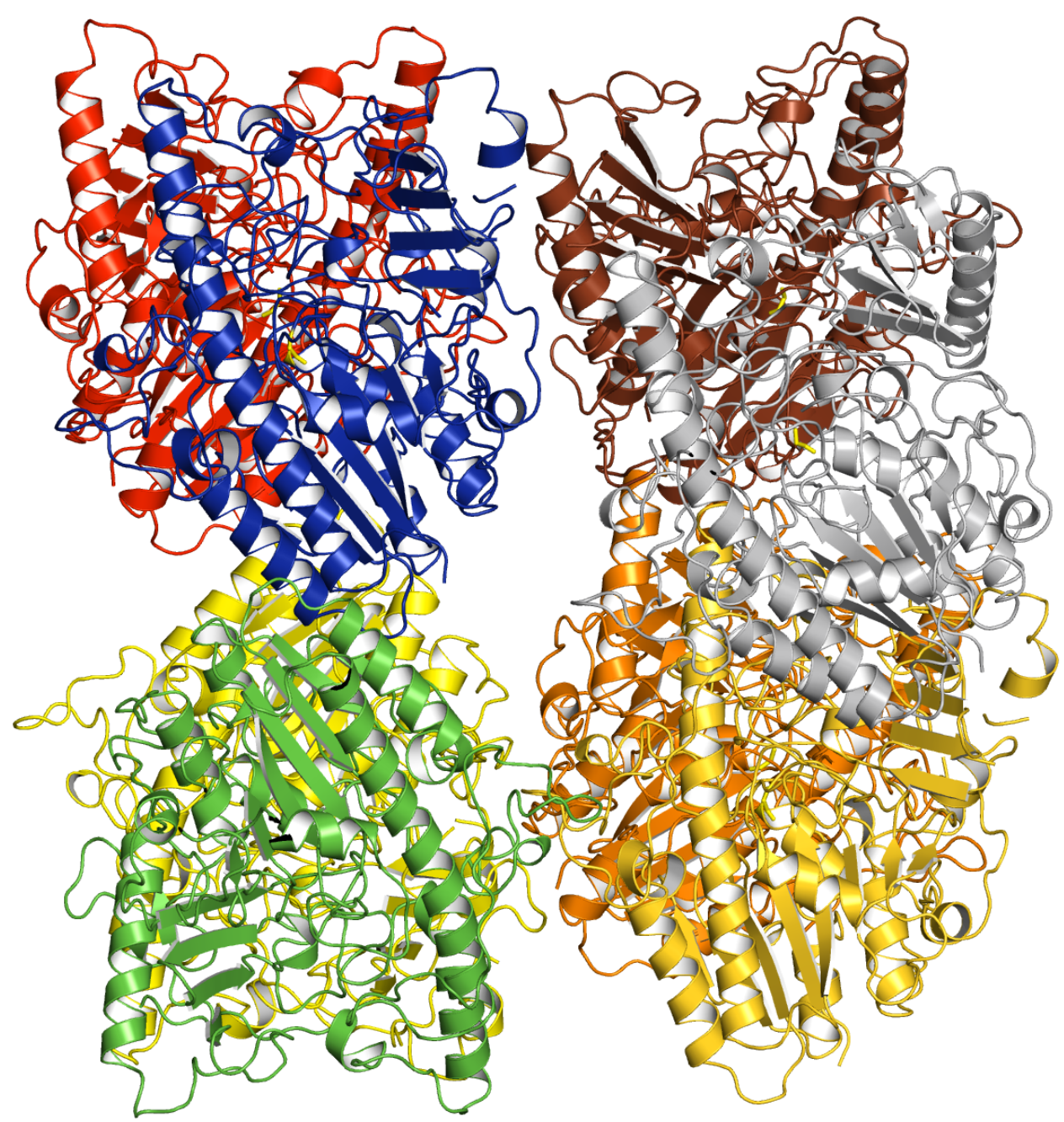

Figure 3.15: SGSH dimers in crystal form L. The 8 monomers forming the 4 dimers in the asymmetric unit of crystal form $\mathrm{L}$ are colored differently to show their approximate relative arrangement. 


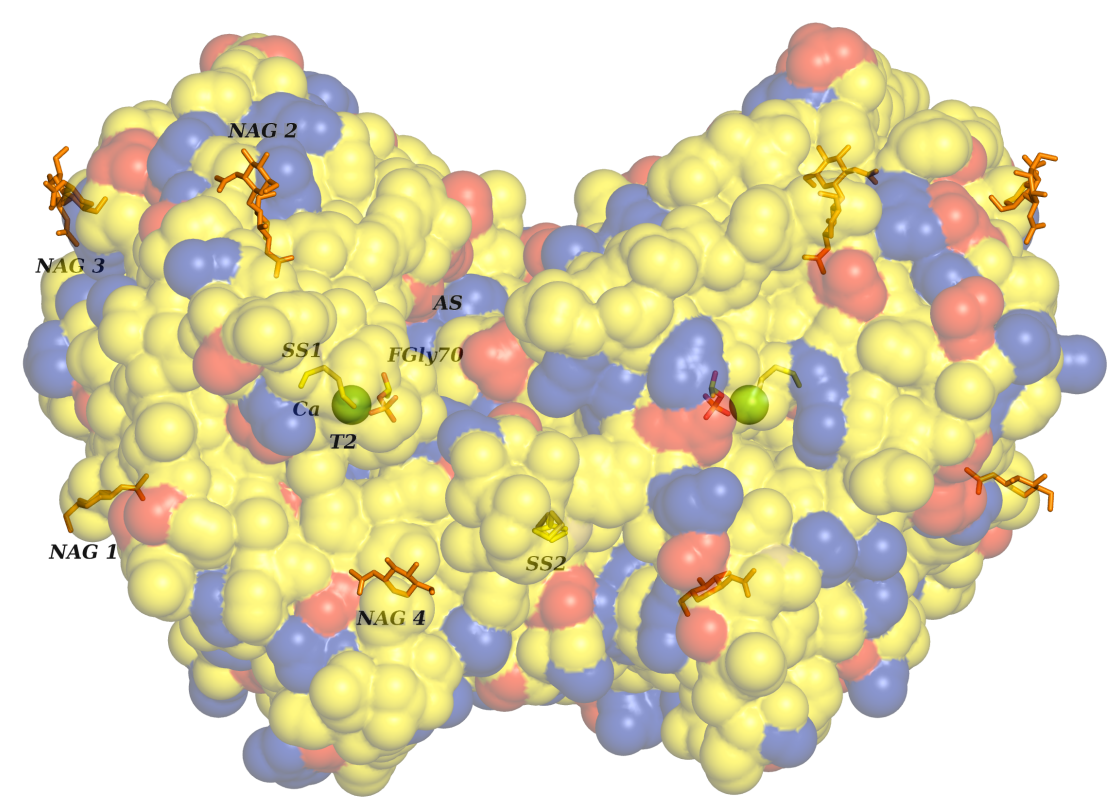

Figure 3.16: SGSH dimer in front surface view with active site mapped onto the enzyme surface. The active site, with the formylglycine (FGly70) and $\mathrm{Ca}^{2+}$ (green ball, labeled as Ca), lies at the bottom of a surface cleft (labeled AS). The entry to the pocket is hidden in this view behind the second $3_{10}$ helix (labeled T2). Nitrogen and oxygen atoms of charged residues (Arg, Lys, His, Asp, Glu) are shown as blue and red balls, respectively; other atoms as yellow balls. Labels are given only for one subunit in the dimer. Glycosylations are numbered in sequence of their occurrence in the chain (NAG 1 through 4). The disulfide bridges are similarly labeled as SS1 and SS2.

a sulfate-binding site. The general location of the active site in the monomer is comparable to that in other arylsufatases.

In terms of secondary structure, active site residues approximately lie in loops at the C-terminal ends of strands $\beta 1$ (Asp31, Asp32), $\beta 5$ (His 181) and $\beta 6$ (Asp273, Asn274) and the N-terminal end of helix $\alpha 2$ (formylglycine [FGly] 70). Overall, the active site lies in a seat cushioned by loops (Fig. 3.18), at the bottom of which seat lie ordered secondary structure elements such as $\alpha$-helices and $\beta$-strands.

Fig. 3.19 shows the active site as viewed from the pocket that leads into it. The divalent metal binding site lies at the front wall, with FGly70 to the right from it. Around this center is placed a rim of positively charged residues.

At the Fly70 position, electron density consistent with a phosphorylated FGly residue was modeled as such. It is stabilized by many interactions, namely with $\mathrm{Ca}^{2+}$, and with the side chains of Arg74, Lys123, His125, His181 and Arg282.

In the divalent metal-binding site, electron density consistent with the pres- 


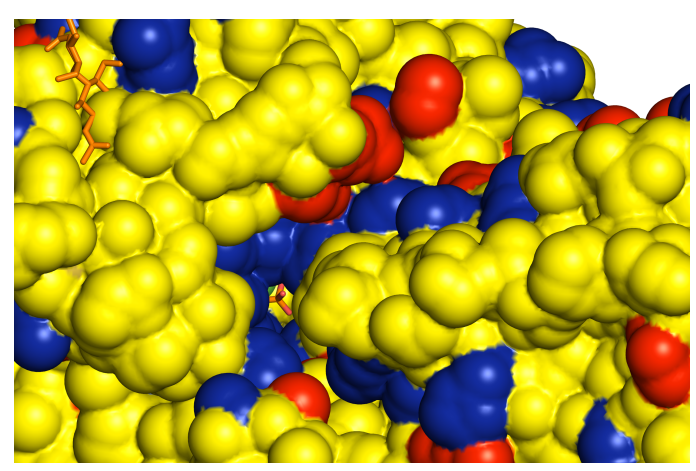

Figure 3.17: Active site pocket in surface cleft. Part of FGly70 (stick model, center) is visible in the active site pocket. The entry to the active site is rimmed by residues with positively charged side chains (blue). Representation as in previous figure. This view was achieved by rotating the dimer in the front view by approximately 45 degrees clockwise as viewed from the top.

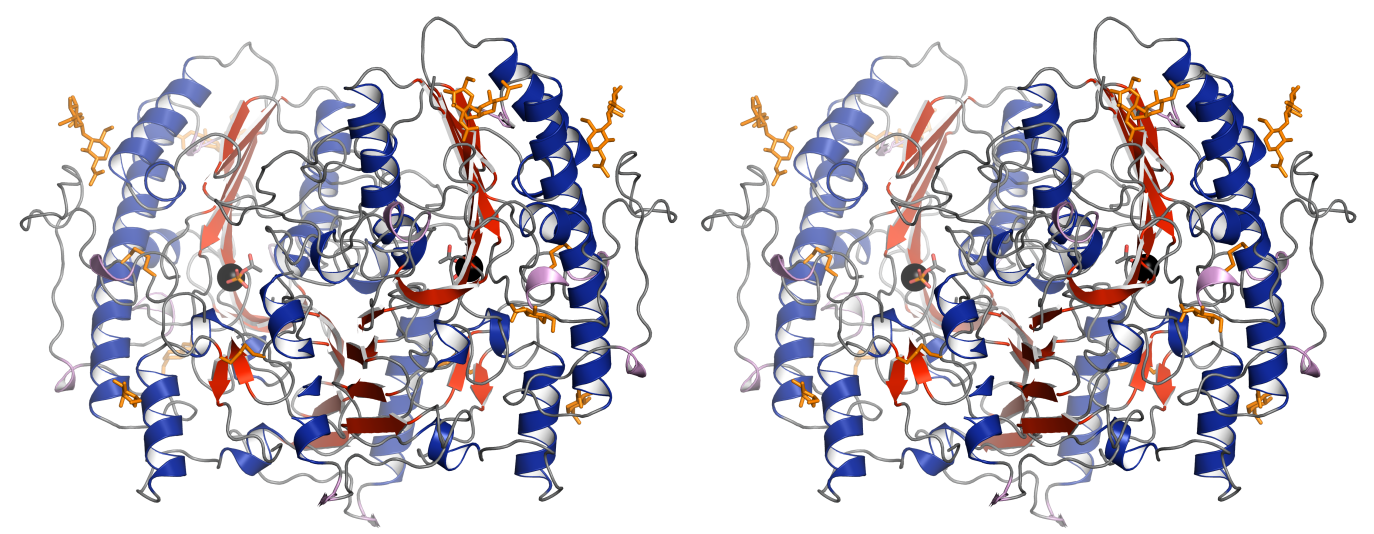

Figure 3.18: Stereo view of the active site pocket showing its location in relation to secondary structure elements. The active site sits cushioned by loops, which are highly variable in different sulfatases (discussed below). 

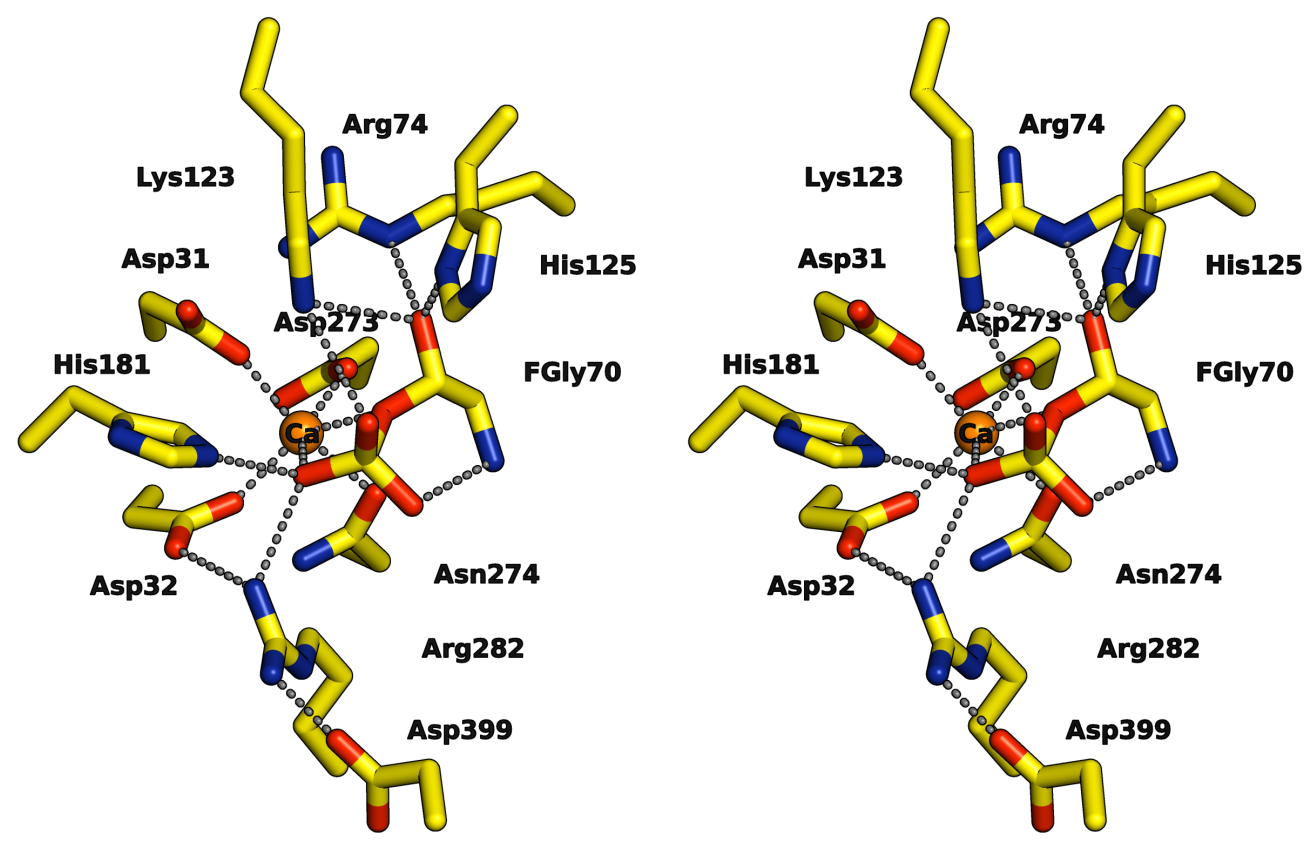

Figure 3.19: Stereo view of the active site, as seen from the entry into it from the cleft. Major interactions are shown and residues labeled. Protein carbon atoms are in yellow, oxygens in red, nitrogens in blue and calcium ion as an orange ball labeled "Ca". The phosphate attached to the FGly side chain faces the entry to the active site pocket. A similar figure first published in Sidhu et al. (2014); copyright IUCr; used with permission. 
ence of a metal ion became slowly apparent during refinement. It was modeled initially as $\mathrm{Mg}^{2+}$ but later as $\mathrm{Ca}^{2+}$ based on bond valence method calculations (Müller et al., 2003) performed manually and using implementation in XPRO (Sheldrick, 2013). The metal ion is bound in a distorted, approximately octahedral arrangement by six oxygen atoms, one each from the side chains of residues Asp31, Asp32, Asp273 and Asn274, and two from the phosphorylated FGly70.

As shown by Table 3.2 , the active site is highly conserved between human sulfamidase and the specific O-sulfatases (excluding BcPMH) with known 3dimensional structures: 9 of 10 active site residues are strictly conserved between them.

A notable exception is Arg282 in sulfamidase. A lysine is found in its place in all of these O-sulfatases, including BcPMH. On the other hand, a protein database search using BLAST revealed that all of these 10 important active site residues, including the arginine, appear to be highly conserved amongst human sulfamidase and its closest eukaryotic sulfamidase homologues (highlighted in a comparison shown in Fig. 3.20; numbering shown for human sulfamidase) ranging from vertebrates to invertebrates such as an ant.

\subsubsection{Comparison with O-sulfatases}

The above-mentioned 5 O-sulfatases, which catalyze the hydrolysis of an S-O bond in sulfate esters, with previously described 3-dimensional structures were found to share only a low sequence identity of 19 to $23 \%$ with sulfamidase, as determined using the PROMALS3D server. These are referred to below as specific $\mathrm{O}$-sulfatases to distinguish them from a non-specific homologue that has also been described. It is the sulfatase/hydrolase from Burkholderia caryophilli PG2952 (BcPMH) (van Loo et al., 2010). This enzyme is a hydrolase/sulfatase that acts on a relatively diverse range of substrates including monoesters of phosphate, phosphonate, sulfate and sulfonate, and di- and triesters of phosphate.

Fig. 3.21 shows a comparison of the three-dimensional structures of a subunit each of sulfamidase and its closest O-sulfatase homologues with known structures. The overlay shows that these $\mathrm{N}$ - and O-sulfatase structures can be approximately divided into two halves: one that superimposes relatively well amongst them (the conserved part) and the other that does so relatively poorly (the nonconserved part). The conserved part is rich in ordered secondary structure elements such as $\alpha$ helices and $\beta$ strands; the unconserved one is rich in loops. The active site lies in the conserved part but at the border with the nonconserved part. The pocket leading into the active site thus traverses the nonconserved, loop-rich variable part.

Fig. 3.22 illustrates that within what has been broadly classified as the conserved part above, however, there are significant differences in the presence and/or positioning of a significant number of decorating $\alpha$-helices and loops that sur- 


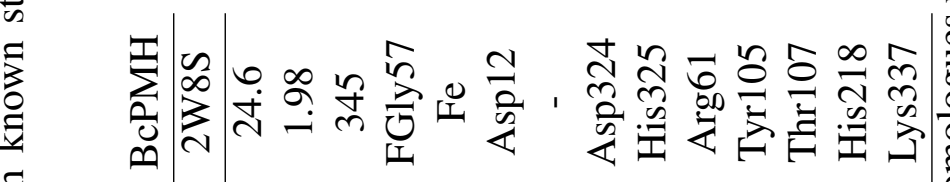

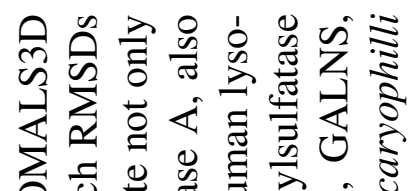

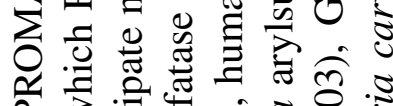

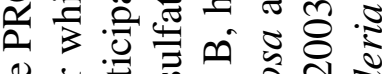

莺

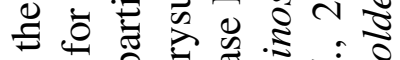

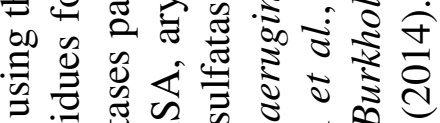

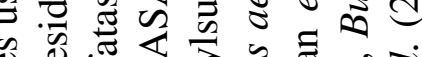

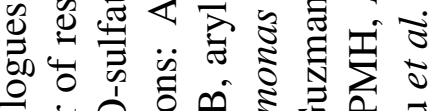

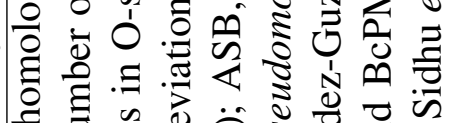

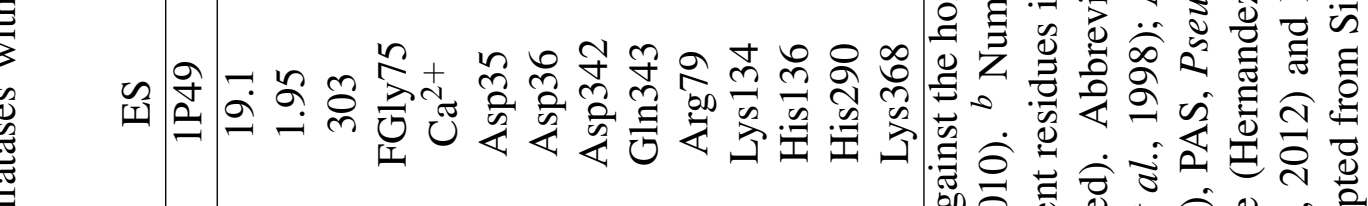

䒿

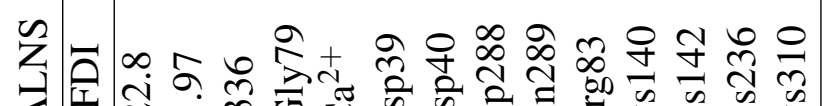

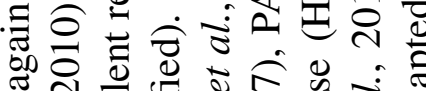

㲾

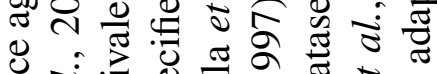

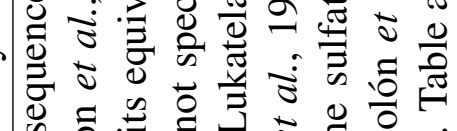

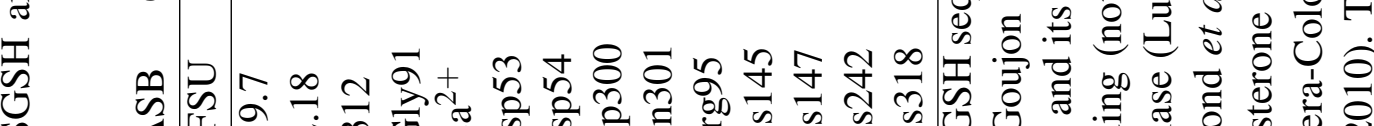

《I

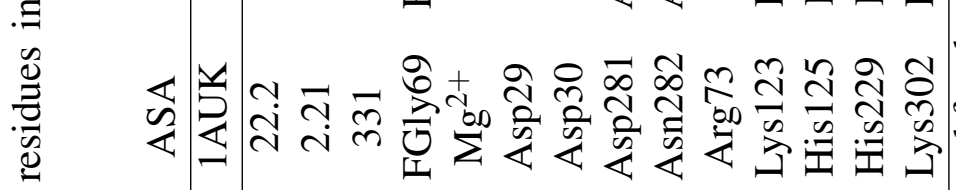

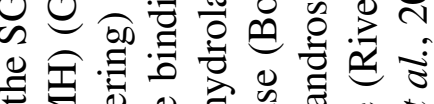

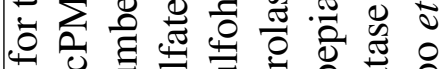

$\stackrel{Ð}{=}$

:

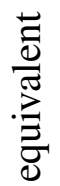

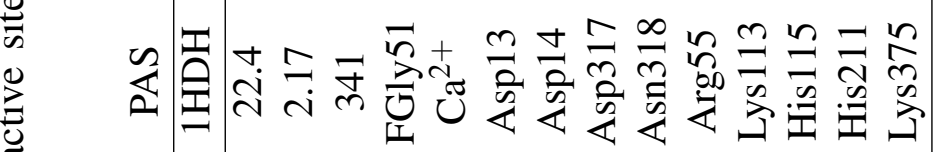

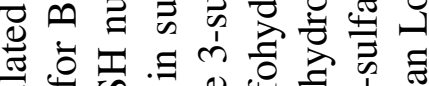

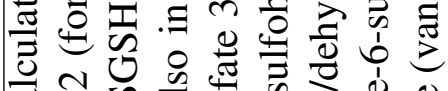

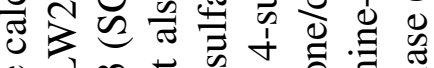

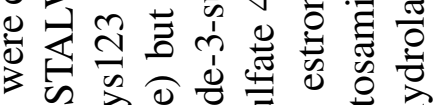

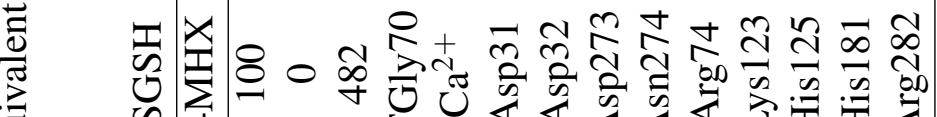

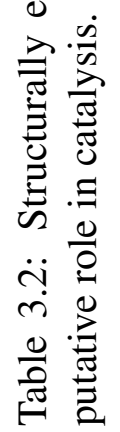

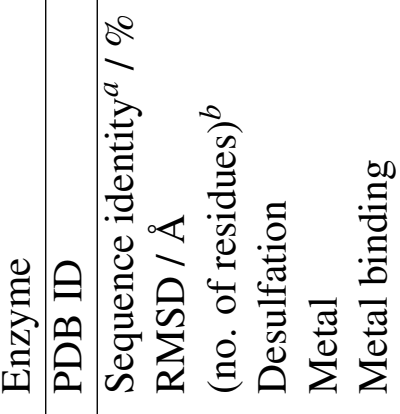

告

.

๑ง

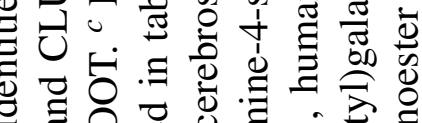

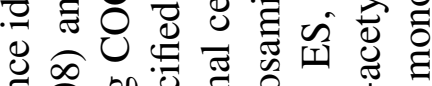
象

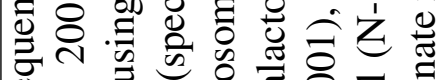
棺 它

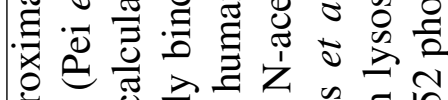

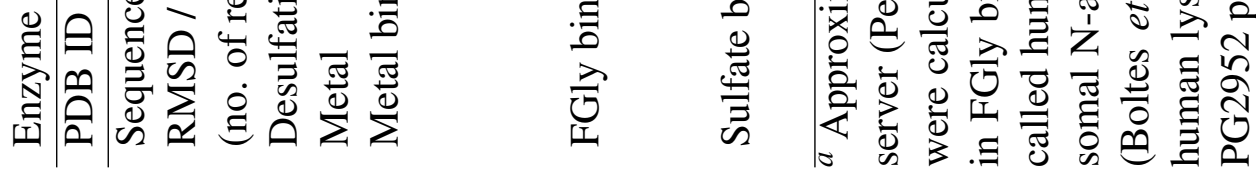



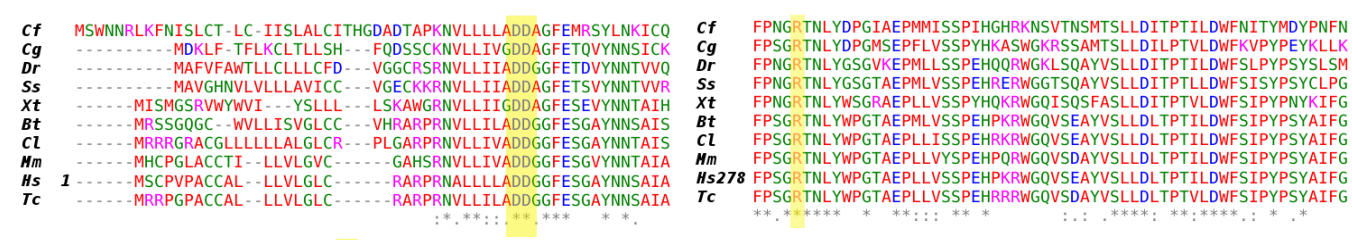

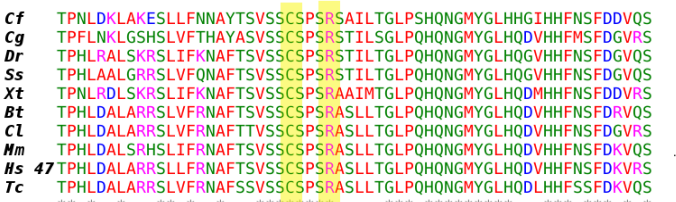

cf LPRILKKNNIRTGIIGKKHVGNKVYPFDFAHTEENNSILQVGRNITYIKLLVREFLSVQ

LPLLLKRANTHTGI IGKKHVGPGPVYPFDFAYTEETNSVLOVGRNITKTKL LVKFFOSH

LPLLLGQANIRTGIIGKKHVGPGPVYPFDFAYTEENNSVLQVGRNITRIKLLVRKFFQTQ

LPLILRQAGIRTGIIGKKHIGPESVYPFDFSYTEENSSVLQVGRIITRIKLLVRKFLQS -

LPLLORAGVTGITGKKHVGPSVYPFEFAHTEENSSVLOVGRITRIKLVAKFLOT-

LPLLLNOAGVRTGIIGKKHVGPETVYPEDFAFREENSSYMOVGRUTRTKOLVOKFEOT-

Hs107 LPLLLSAAGVRTGIIGKKHVGPETVYPFDFATTEENGSVLOVGRNITRIRLLVRKLQT
Ic LPLLLQAGVRTGI IGKKHVGPEAVFPFDFAYTEENGSVLOVGRITRIKLLVRKLQT

cf $\ldots$-..-NKTQPFFLYIAFHDPHRCGHTNPEYGNFCEKFGNSD-VGMGTIPDWINPIYYQW

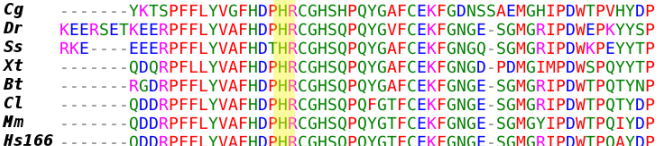

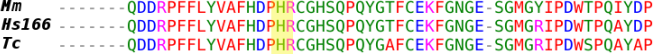

tc FPSGRTNLYWPGTAEPLLVSSPEHRRRWGQVSDAYVSLLDLTPTVLDWFSIPYPSYAIFG

cf EQVKVPYYVQNTEAARRDIAAQYTTISRLDKGVGLVLEELKNAGFKDNTLVIYTSDNGIP

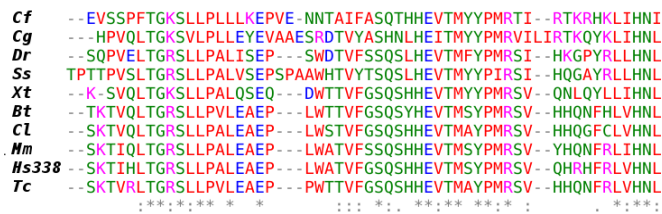

年

cf NYRMPFPIDQDFYISPTFQDILNRTQTHQSLPWYKTLKKYYQRPEWELYDLKYDPEELNN

HYRMPFPIDQDFYISPTFQDLLNRTQSGRPTGWFKTLNEYYYRQRWELFDVRTDPMEKVN NFKMPFPIDQDFYVSPTFODLLNRTASGOPTSWFKTLHYYYYRDRWELYDRSADISEIK N HFKMPFPIDQDFYISPTFQDLLNRTTAGQPTGWYKDLHOYYYRERWELYDRNQDPHETHN DLLNRTVAGHPTGWYKDLRHYYYRERWELYDRSQDPETN

Hs391 NFKMPFPIDQDFYVSPTFQDLLNRTTAGOPTGWYKDLRHYYYRARWELYDRSRDPHETON

Tc NFKMPFPIDQDFYVSPTFQDLLNRTVAGRPTGWYKDLHHYYYRDRWELYDQSRDPHETHN

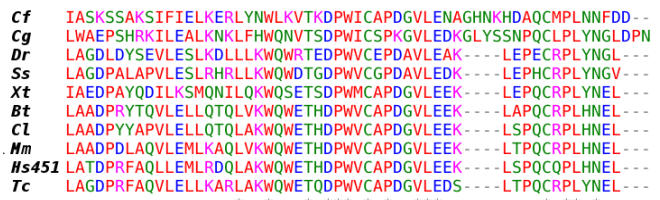

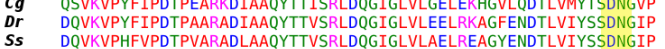

$x t$ EQVQVPYFIQDTPSARKDIAAQYTTIGRMDQGIGLVLSELYNAGHENDTLVIFSSDNGIP

KDVVVPYFVPDTPAARADLAAOYTTIGRMDOGIGLVLELRGAGVNDTLVIFSDNGEP

QDVMVPYFVPDTPAARADLAAQYTTIGRMDQGVGLVLQELRGAGVLNDTLIIFTSDNGIP

LDVLVPYFVPNTPAARADLAAQYTTVGRMDQGVGLVLQELRDAGVLNDTLVIFTSDNGIP

DVLVYFIPDTPAARADLAAQYTTI GRIDQGVGLVQELRGGVLNDLLVIFTS

Figure 3.20: Amino acid sequence comparison of human sulfamidase with closest related sequences annotated as those of sulfamidases (multiple sequence alignment). Numbering shown is for human sulfamidase. Abbreviations: Cf, Camponotus floridanus (Florida carpenter ant), accession code EFN70845.1; Cg, Crassostrea gigas (Pacific oyster), EKC39134.1; Dr, Danio rerio (zebrafish), NP_001116740.1; Ss, Salmo salar (Atlantic salmon), NP_001133784.1; Xt, Xenopus tropicalis (western clawed frog), NP_001120065.1; Bt, Bos taurus (domestic cow), NP_001095659.2; Cl, Canis lupus familiaris (dog), NP_001003114.1; Mm, Mus musculus (house mouse), AAF29460.1; Hs, Homo sapiens, NP_000190.1; Tc, Tupaia chinensis (Chinese tree shrew), ELW68227.1. Figure published in Sidhu et al. (2014); copyright IUCr; used with permission. 


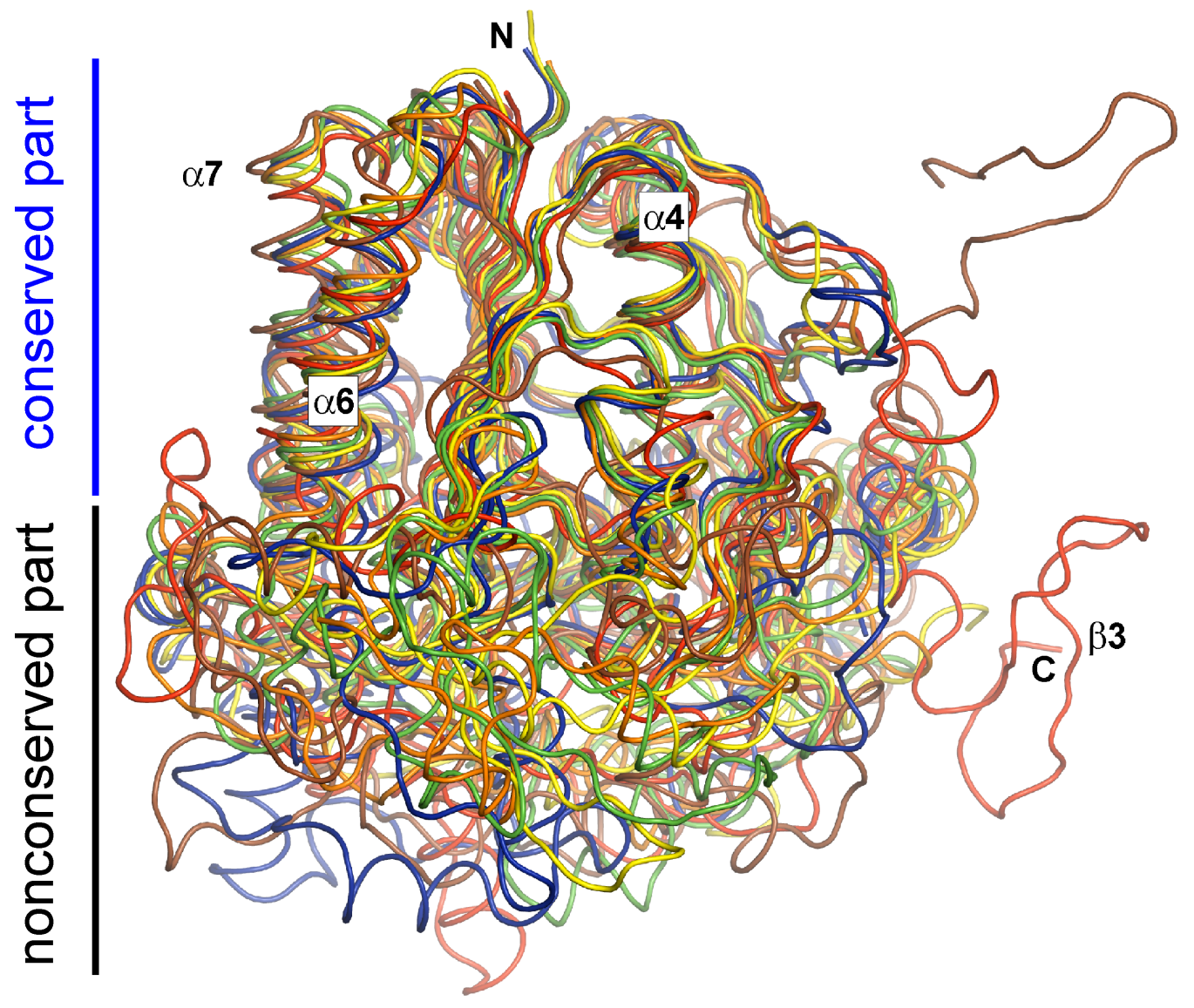

Figure 3.21: Overlay of sulfamidase with related O-sulfatases. About half of the molecule can be superimposed well (labeled "conserved part") onto the related molecules. This part is at the top of the figure and contains most of the ordered secondary structure elements such as helices and beta strands. The other half is rich in loop and highly variable between them ("unconserved part"). Color coding: human sulfamidase (red), ASA (orange), ASB (yellow), PAS (blue), GALNS (green), and BcPMH (brown). Labels for sulfamidase: N-terminus (N); C-terminus (C). Some secondary structure elements are also labeled for orientation; the third beta sheet is labeled $\beta 3$. Figure published in Sidhu et al. (2014); copyright IUCr; used with permission. 

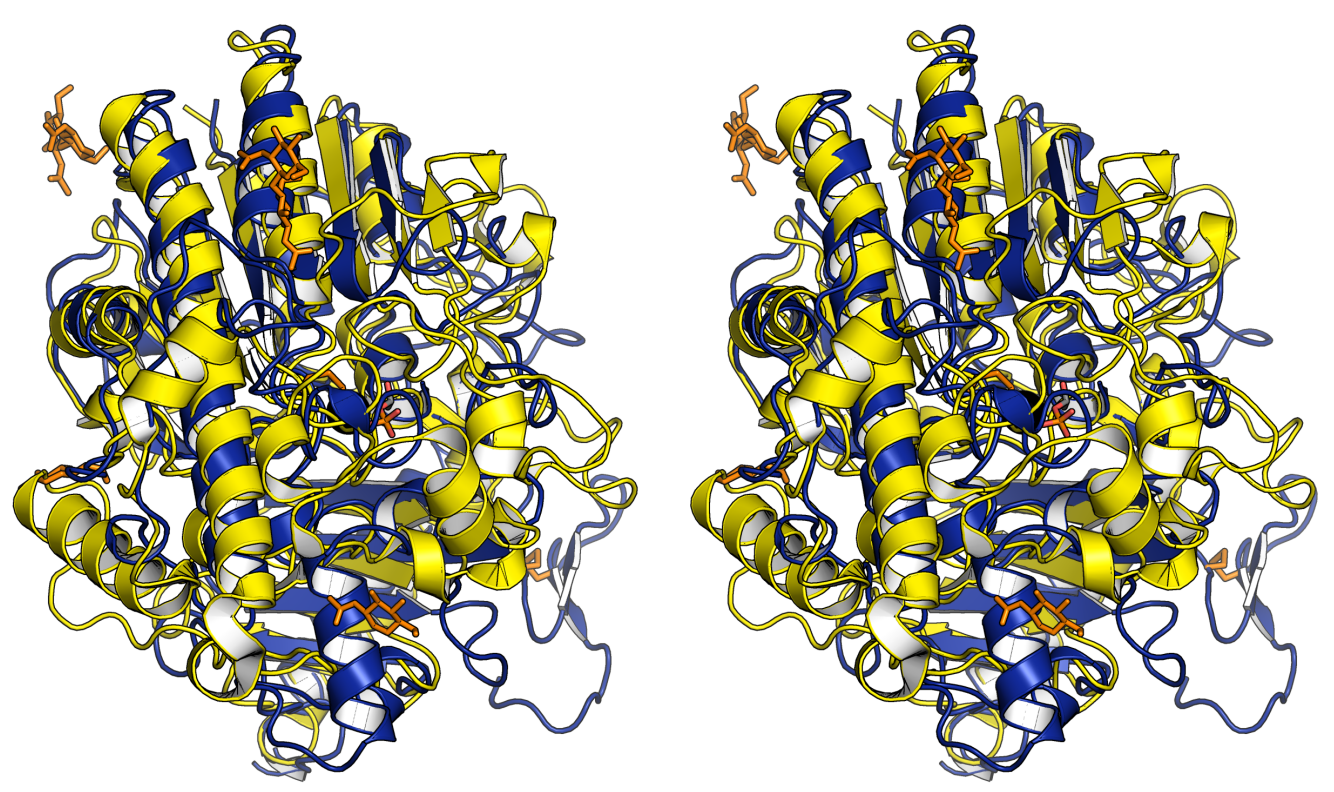

Figure 3.22: Stereo view of a comparison of SGSH with PAS. SGSH is shown in blue, PAS in yellow. Additional structural elements are shown in SGSH for orientation, including FGly70 (sticks), $\mathrm{Ca}^{2+}$ (black ball), glycosylations and disulfide bonds (sticks).

round the core $\beta$-sheets. This is shown by a superposition of a subunit of sulfamidase (blue) with its counterpart from PAS (yellow), the structure from which the search model fragment was derived in the present study to solve the sulfamidase structure. The long helix and its relative displacement, which was one barrier to overcome in solving the structure, is seen (on the left, view corresponding to the front view previously described). Several decorating helices that are present in PAS but either absent or significantly displaced in sulfamidase can be seen in the foreground, along with one that is present in sulfamidase but absent in PAS. The entry to the active site is from the right (roughly from a $50^{\circ}$ angle with the horizontal view direction). It is shown from an appropriately rotated direction in Fig. 3.23 , in which sulfamidase is displayed in a surface representation and PAS in a ribbon one. The sulfamidase active site pocket can be seen at the center (FGly70 as sticks) lying in its cushion of loops (gray). The entry to the pocket contains significant differences between the two enzymes, especially in the placement of loops. 

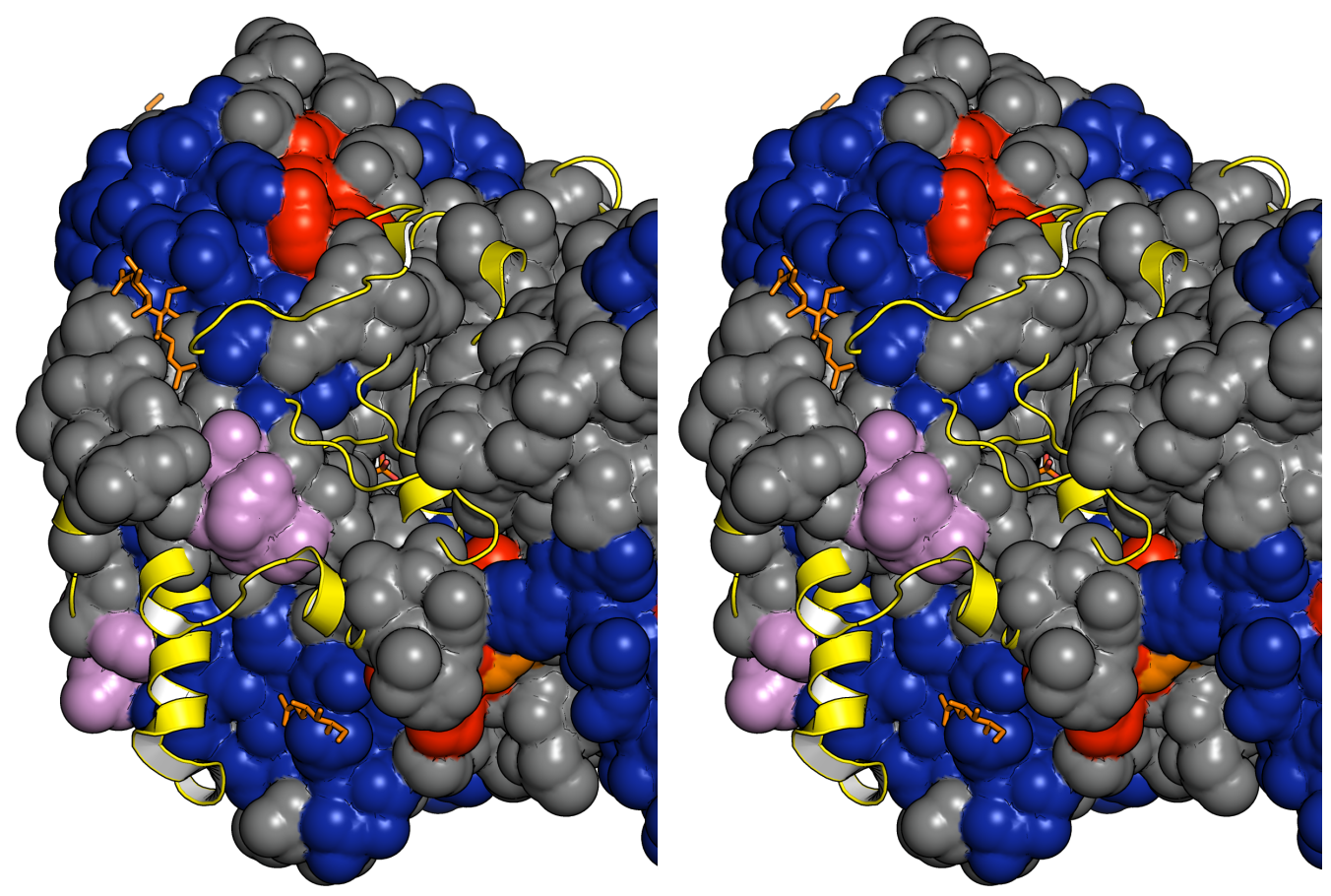

Figure 3.23: Stereo view of a comparison of SGSH with PAS in the active site region. SGSH is shown in surface representation by secondary structure coloring as above (loops gray; helices blue and violet; $\beta$ strands red); PAS is shown in yellow (ribbon diagram). Phosphorylated FGly70 in the active site of SGSH is shown as sticks. 

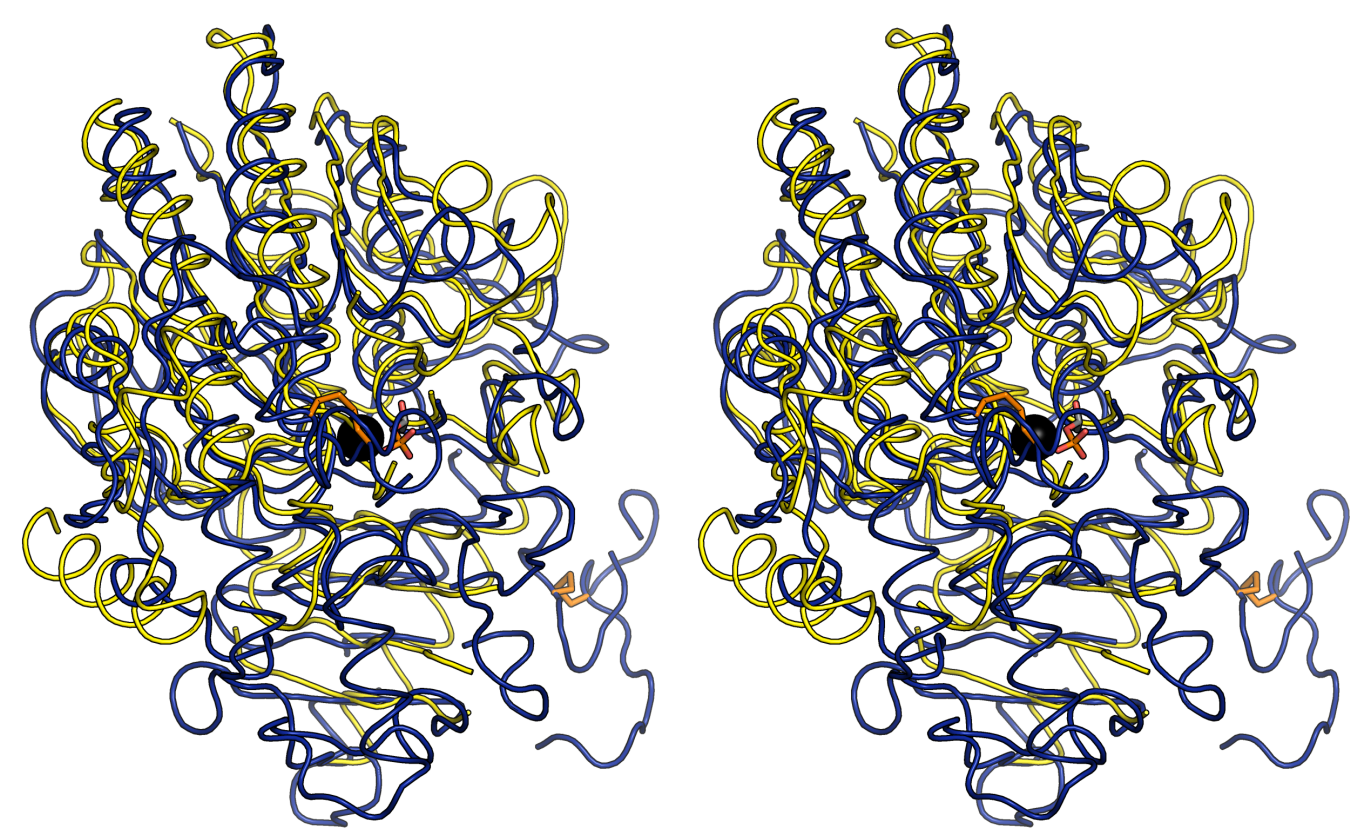

Figure 3.24: Stereo view of a comparison of sulfamidase (blue) with the rotationtranslation solution (yellow) in approximately the front view. Also shown are: the divalent metal ion (black ball), and FGly70 and the disulfide bridges (stick models).

\subsubsection{Comparison of the final model with the rotation-translation solution}

Fig. 3.24 shows a comparison of the final SGSH structure (blue) with that of the rotation-translation solution (yellow) from which it was derived. Although there is a general similarity in the location of secondary structure elements, significant differences exist in the locations or even the presence or absence of a subset of $\beta$-strands and the peripheral helices and loops. The differences are consistent with the difficulty experienced in solving the structure starting from the rotationtranslation solution.

\subsection{Discussion}

\subsubsection{Sulfamidase monomer}

The enzyme monomer has a characteristic sulfatase fold, which belongs to the $\alpha / \beta$ hydrolase fold family (Nardini and Dijkstra, 1999). When overlaid on each other, the 10 monomer chains in the two crystal forms display a low $\mathrm{C}^{\alpha}$ root mean square deviation. This is partly due to local NCS restraints having been 
used in the refinement, including the last cycles. However, the exclusion of these restraints was tested and led to a worsening of figures of merit, including $\mathrm{R}_{\text {free }}$. These observations suggest that the enzyme monomers possess a low degree of difference in overall structure under the two different crystallization conditions, even though the similarity may be overestimated due to the NCS restraints.

\subsubsection{Sulfamidase dimer}

All 10 chains of the enzyme in the two different crystal forms are associated together such that they form 5 homodimers that are related by NCS 2-fold axes. In previous studies, recombinant sulfamidase precursor has been shown to be synthesized as a $62 \mathrm{kDa}$ glycoprotein and then processed to a final product of $56 \mathrm{kDa}$. Active enzyme isolated from human liver has been previously shown to have an apparent molecular weight of approximately $115 \mathrm{kDa}$, with a subunit size of 56 $\mathrm{kDa}$, suggesting that the enzyme exists in vivo as a dimer (Freeman and Hopwood, 1986). The enzyme isolated from human placenta has also been shown to have a molecular weight of approximately $110 \mathrm{kDa}$ (Paschke \& Kresse, 1979).

Sulfamidase is believed to fold into its native conformation in the endoplasmic reticulum. It is then transported via the Golgi network to the lysosome, the resident organelle in vivo for the mature, active enzyme. The crystal structure of the enzyme was solved for two different crystal forms grown at $\mathrm{pH} 7.5$ and 5.1. The enzyme exists as a similarly structured homodimer in both crystal forms. This is consistent with results from a previous study in which no changes in oligomeric state of the enzyme were observed as a function of buffer $\mathrm{pH}$ and ionic strength (Freeman and Hopwood, 1986). Of the residues displaying some interaction at the dimer interface of the 5 dimers, $\mathrm{pH}$-titrable side chains are part of the salt bridge His185-Glu488'. A possible salt bridge close to the interface may also be formed by the Arg428-Glu501' pair. Although the relative position of the monomers in the 5 dimers is slightly flexible (up to approximately $3.0^{\circ}$ ), it is difficult to exclude that this is mainly an artifact of crystal packing.

\subsubsection{Active site}

Electron density consistent with a formylglycine was observed at the position of Cys70 in the active site. Cys70 was shown to play a role in catalysis in SGSH (Daniele and Di Natale, 2001).A formylglycine is present also in the enzyme $\mathrm{BcPMH}$ that displays low substrate and reaction specificities.

Additionally, however, difference density suggestive of a covalently bound phosphate or sulfate group was observed next to the active site formylglycine. This density was modeled as phosphorylated FGly70 as phosphate buffer (20 $\mathrm{mM} \mathrm{K}_{2} \mathrm{HPO}_{4}, \mathrm{pH}$ 7.5) was used in purifying sulfamidase. Phosphate has been 
previously found to be a strong inhibitor of sulfamidase (Karpova et al., 1996). Inhibitory concentration at which $50 \%$ of maximal activity is obtained has been determined to be approximately $1 \mathrm{mM}$ of phosphate (Dr. Krätzner, personal communication). ASA from rabbit liver (Lee and Van Etten, 1975) and human Nacetylgalactosamine-6-sulfatase (Bielicki et al., 1995) have both been shown to be inhibited by sulfate and phosphate. Sulfamidase has also been previously shown to be potently inhibited by sulfate (Freeman and Hopwood, 1986). It can not be excluded, however, that the density is the result of partial occupancy of the site by both phosphate as well as sulfate. The latter could have been picked up by the enzyme during its expression.

Initially $\mathrm{Mg}^{2+}$ had been modeled into the divalent cation-binding site in the active site as the crystallization conditions contained $0.2 \mathrm{M} \mathrm{Mg}^{2+}$. However, bond valence calculations manually and as implemented in XPRO (Sheldrick, 2013) were consistent with the cation being $\mathrm{Ca}^{2+}$ instead and it was then modeled as such. It appears plausible that $\mathrm{Ca}^{2+}$ bound to the enzyme in its intracellular transit during expression. $\mathrm{Ca}^{2+}$ also appears to be the natural cation in sulfatases (Table 3.2 and references therein). However, this does not constitute definitive proof that it is $\mathrm{Ca}^{2+}$. Although the bond valence method has been shown to be able to differentiate between $\mathrm{Ca}^{2+}$ and $\mathrm{Mg}^{2+}$ relatively well, the discrimination could worsen with resolution. The data and resolution also do not make it feasible to exclude the possibility of multiple occupancy at this site by both $\mathrm{Ca}^{2+}$ and $\mathrm{Mg}^{2+}$.

It was previously predicted (Myette et al., 2009), based on an in-silico study, that histidines were not part of the active site in sulfamidase from Flavobacterium heparinum, in contrast to the case with the above-mentioned specific O-sulfatases. Our structure shows that both histidines in the active site of these $\mathrm{O}$-sulfatases are strictly conserved between them and human sulfamidase.

The 3-D structure of approximately half of the molecule is well conserved between these enzymes. The other half is rich in loops and highly variable. The active site lies in the conserved half and is highly conserved between sulfatases and O-sulfatases as also amongst the latter. The entry to the active site, however, traverses the variable part. In this region, sulfamidase shows a high degree of variability from O-sulfatases, as do the latter amongst themselves. It appears plausible that this arrangement allows these enzymes to catalyze a similar reaction on a diverse range of sulfated substrates. As Rivera-Colón et al. (2012) point out, the fact that the deficiency of one sulfatase activity is not compensated for by other sulfatases but leads to disease indicates that sulfatases tend to be relatively specific for their substrates. How is this specificity achieved? The narrowness of the active site region and the cleft has been suggested as one reason for the high substrate specificity of these enzymes, especially in an environment of significant competition with phosphorylated substrates (von Bülow et al., 2001). In addition, the two structurally similar anionic species, phosphate and sulfate, can themselves often 
be well discriminated by enzymes due to differences in the protonation states of these anions at lysosomal pH (Hodel and Quiocho, 2012).

Nine of the 10 important active site residues are highly conserved between sulfamidase and the above-mentioned specific O-sulfatases. The single exception is Asn274 (sulfamidase numbering), which is conservatively replaced by a glutamine in ES (Table 3.2). However, an active site difference between sulfamidase and $\mathrm{O}$-sulfatases that stands out is that a lysine that is conserved in these $6 \mathrm{O}$ sulfatases is replaced in sulfamidases by an arginine (Arg282). Arg282 forms salt bridges and hydrogen bonds with two aspartate residues (Asp32 and Asp399) that lie $7.6 \AA$ apart and on opposite sides of the arginine. It appears that these interactions would be destabilized if the arginine were to be replaced by a lysine. An arginine appears to be highly conserved in sulfamidases. Previous studies have indicated that arginine interacts up to 2.5 times more strongly with heparin than does lysine (Stenlund et al., 2002; Fromm et al., 1995). Taken together, the structure suggests an important role for the sulfamidase Arg282 in substrate binding.

Asp94 is a Ramachandran outlier in all chains in both crystal forms that grew under conditions of different $\mathrm{pH}$ values of 5.1 and 7.5, suggesting that this may not be accidental. It lies $7.6 \AA$ from the active site residue His 125 and forms part of the solvent-exposed surface along the active site cleft. It is conceivable that it plays a role in substrate binding, possibly forming hydrogen bond interactions with a carboxylate on the substrate. Asp94 also participates in forming the dimer interface though, lying $3.5 \AA$ from the side chain of Phe $336^{\prime}$ at the interface. This may also play a role in its position in the Ramachandran plot.

\subsubsection{Enzymatic reaction mechanism}

As mentioned above, human sulfamidase is an $\mathrm{N}$-sulfatase that catalyzes the breaking of the $\mathrm{S}-\mathrm{N}$ bond from $\mathrm{N}$-sulfo-glucosamine at the non-reducing terminal of the linear GAGs heparin and heparan sulfate. In contrast, O-sulfatases catalyze the cleavage of an S-O bond from O-linked sulfate esters. The present structure shows that, despite the low sequence identity between sulfamidase and $\mathrm{O}$-sulfatases and the difference in the type of bond they act on, the active sites of both are highly similar in architecture. Nine of 10 important active site residues are highly conserved betwen sulfamidase and these O-sulfatases, suggesting a shared reaction mechanism and an evolutionary relationship between the two groups of enzymes. Therefore, a reaction mechanism is proposed that is analogous to one of two mechanisms proposed for O-sulfatases (Boltes et al., 2001; von Bülow et al., 2001).

In the machanism proposed, the sulfate group is first passed from the substrate to the enzyme and, in a subsequent part, the enzyme is desulfated in turn (Fig. 3.25). The oxygen atom of hydrated formylglycine (labeled as Step 1) that coordinates $\mathrm{Ca}^{2+}$ is activated, through loss of a proton to a base (B1), so it can launch 


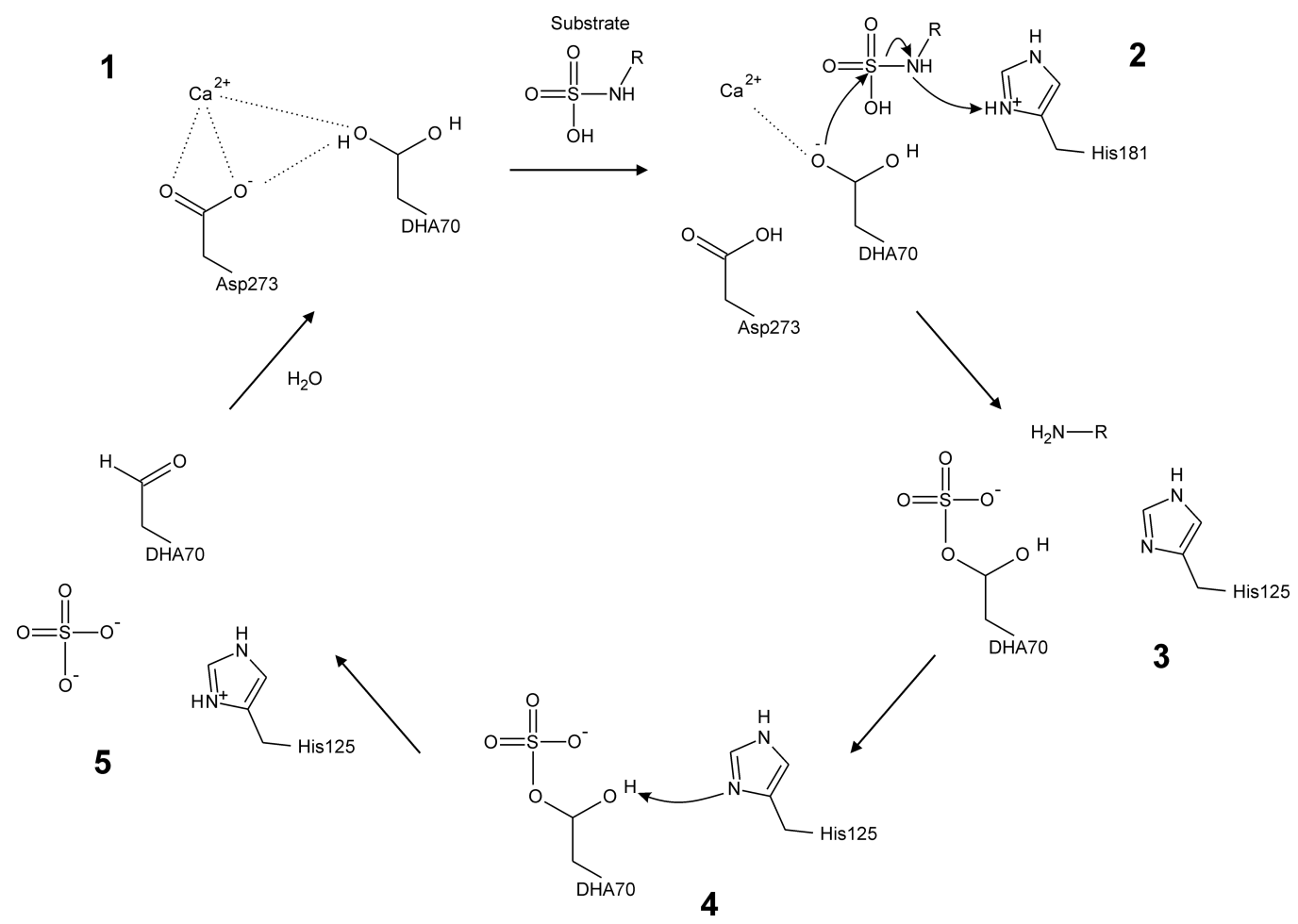

Figure 3.25: Schema of proposed enzymatic reaction mechanism. In the first part, the sulfate group is transferred to the enzyme; in the second part, the enzyme itself is desulfated (discussion in the text). FGly indicated here by its hydrated form, DHA (dihydroxyalanine). This rendering courtesy Dr. R. Krätzner. Figure adapted from Sidhu et al. (2014); copyright IUCr; used with permission.

a nucleophilic attack on the sulfur center of the N-linked sulfate of the substrate (Step 2). The result of the attack is a transition state with a pentavalent sulfur and a covalent linkage of the enzyme and the substrate. The transition state falls apart such that the S-N bond breaks. This is facilitated by the donation of a proton to the leaving amine by an acidic species (A1). This frees the desulfated substrate that can potentially diffuse away, leaving a sulfated enzyme (Step 3).

In the second part, the second hydroxyl group of the hydrated formylglycine is also deprotonated by a base (B2) (Step 4). This results in the formation of a double-bond between the deprotonated hydroxyl oxygen and the $\mathrm{C}_{\beta}$ carbon of FGly70, with the simultaneous breaking of the bond between this $C_{\beta}$ and the other oxygen that bridged it to the sulfur of the sulfate group (Step 5). Thus the enzyme is desulfated, regenerating the formylglycine. It is hydrated, commencing another round of catalysis.

Many candidates have been proposed in PAS for the identity of the acidic 
species A1: Lys375 (PAS numbering), His211, $\mathrm{O}_{\gamma} 2$ via the sulfate group, and a water. It has been plausibly suggested that more than one of these groups could act as acid group A1 depending upon the solution $\mathrm{pH}$ (Boltes et al., 2001). A density functional theory based quantum mechanical study utilizing the coordinates of the crystal structure of PAS (PDB ID 1HDH) suggests that the histidine could serve as the proton donor in that enzyme (Marino et al., 2013). In sulfamidase, the lysine equivalent to the one in PAS is absent; it is replaced by arginine ( $\operatorname{Arg} 282$, sulfamidase numbering), which would be a poor candidate due to its high $\mathrm{pK}_{a}$. However, the histidine is structurally conserved (His 181) and appears to be a good A1 candidate. His181 should also be protonated at lysosomal $\mathrm{pH}$. At a higher $\mathrm{pH}$, a water molecule may also play this role, however. The sulfamidase structure suggests that the residue Asp273 could be a good candidate to act as the base B1.

In analogy with an alternative mechanism that has been proposed for O-sulfatases (Bond et al., 1997), one of the sulfate oxygens on the substrate could attack the FGly70 $\mathrm{C}_{\beta}$ atom. While the mechanism discussed above at length appears to be plausible, the data and structure models presented here do not exclude this alternative mechanism in sulfamidase.

\subsubsection{Glycosylation sites}

Five glycosylation sites have been reported for SGSH at Asn positions 41, 142, 151, 264 and 413 in the protein sequence. Mutagenesis experiments indicate that glycosylations at positions 41 and 151 appeared to be especially important for enzyme stability, lysosomal targeting and function; Asn413 is the least important for function (Di Natale et al., 2001). All of these belong to the consensus sequence Asn-X-Ser/Thr (where $\mathrm{X}$ is any natural amino acid except Pro) that is highly conserved between organisms as diverse as archaea and humans (see Helenius and Aebi, 2001 for a review).

In the present study, electron density corresponding to four glycosylation sites was identified at the solvent-exposed surface next to the side chains of Asn41, Asn151, Asn264 and Asn413. The expected location of the glycosylation sites was confirmed to be the solvent-exposed surface of the molecule. Although ordered density for Asn142 was observed, there was no discernable density corresponding to glycosylation at this site. Possible reasons for the discrepancy include partial glycosylation at this site, a high degree of glycan disorder and genuine differences in glycosylation in the expression systems used in the previous and current studies. 


\subsubsection{Disease-causing mutations}

The structural and mechanistic connection between genotype and phenotype is poorly understood in MPS IIIA (see Yogalingam and Hopwood, 2001 for a review). Genetic, epigenetic and environmental factors contribute to this as well as difficulties assessing signs and symptoms in diagnosis (Beesley et al., 2000; Di Natale et al., 1998; Perkins et al., 1999).

Mutations that have been described to be associated with the disease include nonsense, frameshift and missense mutations. Unless compensated for by the second allele, all of the nonsense and frameshift mutations appear to lead to a severe disease phenotype of MPS IIIA. Approximately 80\% (Pollard et al., 2013) of the mutations in the disease have been found to be missense mutations. Out of the subset of these that have been described, most also lead to a severe disease phenotype in the homozygous or compound heterozygous states. However, some missense mutations are associated with an intermediate or mild (also termed attenuated) phenotype. For instance, some of the most commonly occurring mutations are S66W, R245H, S298P, Q380R and c.1080delC. All of these except S298P are associated with severe disease in homozygous and, as a rule, also in the compound heterozygous form (Valstar et al., 2010, and references therein).

The molecular basis of this variability has been rather poorly understood, however. A major barrier to a better understanding of the effects of the mutations has undoubtedly been that no three-dimensional structure of human, or indeed any, sulfamidase was available. The closest homologues for which the structures had been solved were O-sulfatases. However, they were distant homologues that shared relatively low sequence identity and homology with sulfamidase. Using that structural knowledge to better understand disease mechanism in MPS IIIA was therefore difficult. Interpretation of the effects of mutations based on other sequences and structures was limited to those parts of the sequence that displayed a higher homology. These parts especially included two relatively small signature sulfatase sequence regions (approximately residues 70-80 and 115-124, human sulfamidase numbering).

Table 3.3 lists 80 of the missense mutations associated with the disease. The accompanying figure maps these various mutations to the enzyme structure colorcoded by their described phenotype (Fig. 3.26). The crystal structure of the wildtype glycosylated human enzyme solved in the present study suggests a rational interpretation of the effects of various mutations on enzyme structure and function. Each mutation is classified into a category based on the part of the structure affected (e.g. metal-binding or active site residues, surface or buried residues, etc.). Also listed is the predicted immediate structural effects of the mutation on the enzyme structure as based on the wild-type structure of the enzyme determined in the present study. 
Table 3.3: Missense mutations associated with MPS IIIA

\begin{tabular}{|c|c|c|c|c|c|c|}
\hline Protein & Codon & Phenotype & NASA $(\%)$ & Type & Effect of mutation on structure & Reference \\
\hline M1V & $1 \mathrm{~A}>\mathrm{G}$ & NR & & Signal peptide & Part of signal peptide & 28 \\
\hline L12Q & $35 \mathrm{~T}>\mathrm{A}$ & Mild & & Signal peptide & Part of signal peptide & 26 \\
\hline $\mathrm{A} 30 \mathrm{P}$ & $88 \mathrm{G}>\mathrm{C}$ & NR & 0 & Buried & $\begin{array}{l}\text { Steric clash close to } \mathrm{Ca}^{2+} \text {-binding } \\
\text { site; loss of } \mathrm{H} \text { bond with } \mathrm{Thr} 271\end{array}$ & 28 \\
\hline D32G & $95 \mathrm{~A}>\mathrm{G}$ & Severe & 1 & Metal binding & Disruption of $\mathrm{Ca}^{2+}$ binding & 8 \\
\hline D32E & $96 \mathrm{C}>\mathrm{A} / \mathrm{G}$ & Mild & 1 & Metal binding & Altered $\mathrm{Ca}^{2+}$ binding & 23 \\
\hline G33R & $97 \mathrm{G}>\mathrm{A}$ & NR & 4 & Buried & $\begin{array}{l}\text { Introduces bulky side chain next to } \\
\mathrm{Ca}^{2+} \text {-binding Asp } 32\end{array}$ & 28 \\
\hline Y40N & $118 \mathrm{~T}>\mathrm{A}$ & Interm. & 13 & Surface & $\begin{array}{l}\text { Loss of H bonding to Leu } 294 \text { and } \\
\text { Phe } 60 \text {, and of } \pi \text {-stacking next to } \\
\text { glycosylation site }\end{array}$ & 6 \\
\hline N42K & $126 \mathrm{C}>\mathrm{A}$ & Severe & 6 & Surface & $\begin{array}{l}\text { Loss of } \mathrm{H} \text { bond with Ala } 44, \text { Tyr } 240, \\
\text { Ile45; steric clash }\end{array}$ & 13 \\
\hline A44T & $130 \mathrm{G}>\mathrm{A}$ & Severe & 31 & Surface & Steric clash at surface site & 6,9 \\
\hline S66W & $197 \mathrm{C}>\mathrm{G}$ & Severe & 2 & Buried & Bulky sc in buried position & $2,4,6,7,8,12,25,26,27,28$ \\
\hline $\mathrm{R} 74 \mathrm{C}$ & $220 \mathrm{C}>\mathrm{T}$ & Severe & 0 & Buried & $\begin{array}{l}\text { Disruption of ion pairs or } \mathrm{H} \text { bonds } \\
\text { with } \mathrm{Ca}^{2+} \text { binding residues Asp } 31 \text {, } \\
\text { Asp273, and FGly } 70 \text {; possible in- } \\
\text { terference with disulfide bridge for- } \\
\text { mation }\end{array}$ & $3,4,6,8,9,15,18,23,25,26,27,28$ \\
\hline $\mathrm{R} 74 \mathrm{H}$ & $221 \mathrm{G}>\mathrm{A}$ & Severe & 0 & Buried & $\begin{array}{l}\text { Disruption of ion pairs or } \mathrm{H} \text { bonds } \\
\text { with } \mathrm{Ca}^{2+} \text { binding residues Asp31, } \\
\text { Asp273, and FGly70 }\end{array}$ & 3,12 \\
\hline T79P & $235 \mathrm{~A}>\mathrm{C}$ & Severe & 0 & Buried & $\begin{array}{l}\text { Disruption of } \mathrm{H} \text { bonding with } \\
\text { Ala75, Ser76, Leu81 }\end{array}$ & $4,8,28$ \\
\hline $\mathrm{H} 84 \mathrm{Y}$ & $250 \mathrm{C}>\mathrm{T}$ & Severe & 1 & Buried & $\begin{array}{l}\text { Loss of } \mathrm{H} \text { bonds to Ser } 364 \text { and } \\
\text { Thr } 475 \text {; steric clash in buried posi- } \\
\text { tion }\end{array}$ & 8 \\
\hline Q85R & $254 \mathrm{~A}>\mathrm{G}$ & Severe & 3 & Buried & Steric clash & 7,12 \\
\hline M88T & $263 \mathrm{~T}>\mathrm{C}$ & NR & 0 & Buried & $\begin{array}{l}\text { Destabilizes van der Waals interac- } \\
\text { tions in buried position; clash }\end{array}$ & 21 \\
\hline G90R & $268 \mathrm{G}>\mathrm{A}$ & Severe & 0 & Buried & $\begin{array}{l}\text { Gain of bulky sc in buried position; } \\
\text { change of } \phi / \psi \text { angles }\end{array}$ & 3,25 \\
\hline S106R & $318 \mathrm{C}>\mathrm{A}$ & Mild & 0 & Buried & $\begin{array}{l}\text { Loss of } \mathrm{H} \text { bonds to Leu109, } \\
\text { Val131; clash possibly accommo- } \\
\text { dated within longer loop that is } \\
\text { partly surface-exposed }\end{array}$ & 18 \\
\hline $\mathrm{T} 118 \mathrm{P}$ & $352 \mathrm{~A}>\mathrm{C}$ & NR & 0 & Buried & $\begin{array}{l}\text { Loss of } \mathrm{H} \text { bonds to Asp135; desta- } \\
\text { bilizes } \beta \text { sheet }\end{array}$ & 24 \\
\hline G122R & $364 \mathrm{G}>\mathrm{A}$ & Interm. & 1 & Buried & $\begin{array}{l}\text { Introduction of bulky side chain in } \\
\text { buried position; Gly } \phi / \psi \text { angles }\end{array}$ & $3,6,8,26,28$ \\
\hline P128L & $383 \mathrm{C}>\mathrm{T}$ & Mild & 22 & Surface & $\begin{array}{l}\text { Favorably surface-exposed to min- } \\
\text { imize steric clash; in loop with } \\
\text { FGly-binding His } 125 \text { and Lys } 123\end{array}$ & 6,16 \\
\hline V131M & $391 G>A$ & Severe & 1 & Buried & $\begin{array}{l}\text { Introduces bulky sc in buried posi- } \\
\text { tion; destabilizes loop with FGly- } \\
\text { binding residues }\end{array}$ & 4 \\
\hline $\mathrm{T} 139 \mathrm{M}$ & $416 \mathrm{C}>\mathrm{T}$ & Severe & 1 & Buried & $\begin{array}{l}\text { Introduces bulky sc in buried posi- } \\
\text { tion; loss of } \mathrm{H} \text { bond with Glu141 }\end{array}$ & 4 \\
\hline
\end{tabular}




\begin{tabular}{|c|c|c|c|c|c|c|}
\hline Protein & Codon & Phenotype & NASA $(\%)$ & Type & Effect of mutation on structure & Reference \\
\hline L146P & $437 \mathrm{~T}>\mathrm{C}$ & Severe & 11 & Surface & $\begin{array}{l}\text { Loss of } \mathrm{H} \text { bond with Ser144; some } \\
\text { clash at surface; destabilizes he- } \\
\text { lix } \alpha 5 \text {; close to glycosylation site } \\
\text { (Asn151) }\end{array}$ & 6 \\
\hline R150W & $448 \mathrm{C}>\mathrm{T}$ & Severe & 1 & Buried & $\begin{array}{l}\text { Introduction of bulky aromatic sc; } \\
\text { loss of ion pair with Asp179, H } \\
\text { bonding with His } 181\end{array}$ & 8,12 \\
\hline R150Q & $449 \mathrm{G}>\mathrm{A}$ & Severe & 1 & Buried & $\begin{array}{l}\text { Loss of ion pair with Asp179, H } \\
\text { bonding with His181; next to gly- } \\
\text { cosylated Asn151 }\end{array}$ & $3,6,7,12,26$ \\
\hline L163P & $488 \mathrm{~T}>\mathrm{C}$ & Severe & 8 & Buried & $\begin{array}{l}\text { Disruption of hydrophobic interac- } \\
\text { tions, H bond to Val159; clash; } \\
\text { destabilizes helix } \alpha 6\end{array}$ & 18 \\
\hline D179N & $535 \mathrm{G}>\mathrm{A}$ & Severe & 1 & Buried & $\begin{array}{l}\text { Loss of buried salt bridges with } \\
\operatorname{Arg} 150, \operatorname{Arg} 245\end{array}$ & 6,9 \\
\hline P180L & $539 \mathrm{C}>\mathrm{T}$ & Mild & 0 & Buried & $\begin{array}{l}\text { Some steric clash next to active site } \\
\text { residues Asp31 and His } 181\end{array}$ & 26 \\
\hline $\mathrm{R} 182 \mathrm{C}$ & $544 \mathrm{C}>\mathrm{T}$ & Interm. & 4 & Buried & $\begin{array}{l}\text { Loss of ion pair with Asp235, H } \\
\text { bond with Pro277 close to active } \\
\text { site; possible interference with fold- } \\
\text { ing (Cys) }\end{array}$ & 6,26 \\
\hline G191R & $571 \mathrm{G}>\mathrm{A}$ & Severe & 11 & Surface & $\begin{array}{l}\text { Surface-exposed but steric clash } \\
\text { with sc's of Glu195 and Lys196; } \\
\text { Gly } \phi / \psi \text { angles }\end{array}$ & 18,26 \\
\hline F193L & $579 \mathrm{C}>\mathrm{G}$ & NR & 0 & Buried & $\begin{array}{l}\text { Disrupts } \pi \text {-stacking next to active } \\
\text { site loop (His181) }\end{array}$ & 3,11 \\
\hline R206P & $617 \mathrm{G}>\mathrm{C}$ & Mild & 73 & Surface & $\begin{array}{l}\text { R206 has no backbone amide } H \\
\text { bond to lose; close to glycosylated } \\
\text { Asn151; change in } \phi / \psi \text { angles }\end{array}$ & $7,9,12,20$ \\
\hline P227R & $680 \mathrm{C}>\mathrm{G}$ & Severe & 0 & Buried & $\begin{array}{l}\text { Steric clash from bulky substitution } \\
\text { disrupts packing in buried position }\end{array}$ & 6,9 \\
\hline A234G & $701 \mathrm{C}>\mathrm{G}$ & Severe & 51 & Surface & $\begin{array}{l}\text { Unclear; plausibly destabilization } \\
\text { of helix } \alpha 7\end{array}$ & 4 \\
\hline $\mathrm{D} 235 \mathrm{~N}$ & $703 \mathrm{G}>\mathrm{A}$ & Severe & 1 & Buried & $\begin{array}{l}\text { Loss of buried salt bridge with } \\
\text { Arg } 182 \text {, and of } \mathrm{H} \text { bond acceptor }\end{array}$ & 8,13 \\
\hline $\mathrm{D} 235 \mathrm{~V}$ & $704 \mathrm{~A}>\mathrm{T}$ & NR & 1 & Buried & $\begin{array}{l}\text { Loss of buried salt bridge with } \\
\text { Arg182, and } \mathrm{H} \text { bonds with Thr192, } \\
\text { Thr407 }\end{array}$ & 3 \\
\hline $\mathrm{T} 242 \mathrm{~T}$ & $726 \mathrm{C}>\mathrm{T}$ & NR & 0 & Buried & Unclear & 26 \\
\hline $\mathrm{R} 245 \mathrm{H}$ & $734 \mathrm{G}>\mathrm{A}$ & Severe & 0 & Buried & $\begin{array}{l}\text { Loss of buried salt bridge with } \\
\text { Asp179 and } \mathrm{H} \text { bonds with it and } \\
\text { with Cys } 194 \text {; clash; packing of he- } \\
\text { lix } \alpha 7\end{array}$ & $2,3,4,5,8,18,23,26,27,28$ \\
\hline D247H & $739 \mathrm{G}>\mathrm{C}$ & NR & 1 & Buried & $\begin{array}{l}\text { Loss of } \mathrm{H} \text { bonding with Leu50; } \\
\text { clash }\end{array}$ & 26 \\
\hline G251A & $752 \mathrm{G}>\mathrm{C}$ & Mild & 13 & Surface & $\begin{array}{l}\text { Some clash with sc of His } 49 \text { in } \\
\text { surface-exposed site }\end{array}$ & 23,27 \\
\hline $\mathrm{D} 273 \mathrm{~N}$ & $817 \mathrm{G}>\mathrm{A}$ & Severe & 2 & Metal-binding & Destabilizes $\mathrm{Ca}^{2+}$ binding & 8 \\
\hline Y286S & $857 \mathrm{~A}>\mathrm{C}$ & NR & 4 & Buried & $\begin{array}{l}\text { Disruption of } \mathrm{H} \text { bond with Glu437 } \\
\text { and of } \pi \text {-stacking interactions }\end{array}$ & 11 \\
\hline P288S & $862 \mathrm{C}>\mathrm{T}$ & Severe & 5 & Buried & $\begin{array}{l}\text { Possibly unsatisfied } \mathrm{H} \text { bonding in } \\
\text { sc of Ser in buried position }\end{array}$ & 15 \\
\hline P288L & $863 \mathrm{C}>\mathrm{T}$ & NR & 5 & Buried & Steric clash & 28 \\
\hline E292K & $874 \mathrm{G}>\mathrm{A}$ & Mild & 0 & Buried & $\begin{array}{l}\text { Buried water might offer space to } \\
\text { accommodate larger sc }\end{array}$ & 25 \\
\hline
\end{tabular}




\begin{tabular}{|c|c|c|c|c|c|c|}
\hline Protein & Codon & Phenotype & NASA $(\%)$ & Type & Effect of mutation on structure & Reference \\
\hline P293T & $877 \mathrm{C}>\mathrm{A}$ & NR & 3 & Buried & $\begin{array}{l}\text { Steric clash; loss of Pro from 3- } \\
\text { residue loop }\end{array}$ & 22 \\
\hline P293S & $877 \mathrm{C}>\mathrm{T}$ & Severe & 3 & Buried & $\begin{array}{l}\text { Unclear; loss of Pro in 3-residue } \\
\text { loop }\end{array}$ & 13,28 \\
\hline S298P & $892 \mathrm{~T}>\mathrm{C}$ & Mild & 1 & Buried & $\begin{array}{l}\text { Loss of } \mathrm{H} \text { bonds with Glu300, } \\
\text { His } 301 \text {, but steric clash milder } \\
\text { as buried water offers substitution } \\
\text { space; favorable } \phi / \psi \text { angles (S297, } \\
\text { S298) }\end{array}$ & $3,8,18,23,26,27,28$ \\
\hline E300V & $899 \mathrm{~A}>\mathrm{T}$ & Severe & 48 & Surface & $\begin{array}{l}\text { Loss of surface salt bridge with } \\
\text { Arg23; little steric clash; unclear }\end{array}$ & 19 \\
\hline R304L & $911 \mathrm{G}>\mathrm{T}$ & NR & 7 & Surface & $\begin{array}{l}\text { Loss of surface salt bridge with } \\
\text { Glu355, and of } \mathrm{H} \text { bonds with } \\
\text { Ala351 and Gln307; some steric } \\
\text { clash }\end{array}$ & 22,28 \\
\hline Q307P & $920 \mathrm{~A}>\mathrm{C}$ & Severe & 38 & Surface & $\begin{array}{l}\text { Loss of surface } \mathrm{H} \text { bonds with } \\
\text { Arg304; steric clash; destabiliza- } \\
\text { tion of strand } \beta 8\end{array}$ & 19 \\
\hline A311D & $932 \mathrm{C}>\mathrm{A}$ & NR & 3 & Buried & $\begin{array}{l}\text { Introduction of steric clash, buried } \\
\text { charge, and unsatisfied } \mathrm{H} \text { bonding }\end{array}$ & 28 \\
\hline D317H & $949 \mathrm{G}>\mathrm{C}$ & NR & 4 & Buried & $\begin{array}{l}\text { Steric clash; loss of } \mathrm{H} \text { bonds with } \\
\text { Ser314, Arg346 }\end{array}$ & 28 \\
\hline T321A & $961 \mathrm{~A}>\mathrm{G}$ & NR & 0 & Buried & $\begin{array}{l}\text { Loss of } \mathrm{H} \text { bonds with Asp317, } \\
\text { Leu348 and of van der Waals inter- } \\
\text { actions }\end{array}$ & 3 \\
\hline $\mathrm{I} 322 \mathrm{~S}$ & $965 \mathrm{~T}>\mathrm{G}$ & Mild & 0 & Buried & $\begin{array}{l}\text { Loss of van der Waals interactions, } \\
\text { but I322S can H-bond with Leu318 }\end{array}$ & 8 \\
\hline S347Y & $1040 \mathrm{C}>\mathrm{A}$ & NR & 15 & Surface & $\begin{array}{l}\text { Bulky aromatic in solvent-exposed } \\
\text { position, but with minimal steric } \\
\text { clash; loss of H bonds with Asp324, } \\
\text { Leu349 }\end{array}$ & 26 \\
\hline S347F & $1040 \mathrm{C}>\mathrm{T}$ & Mild & 15 & Surface & $\begin{array}{l}\text { Bulky aromatic in solvent-exposed } \\
\text { position, but with minimal steric } \\
\text { clash; loss of H bonds with Asp324, } \\
\text { Leu349 }\end{array}$ & 14 \\
\hline A354P & $1060 \mathrm{G}>\mathrm{C}$ & Severe & 58 & Surface & $\begin{array}{l}\text { Loss of } \mathrm{H} \text { bond to Pro350; steric } \\
\text { clash with Pro350; change in } \phi / \psi \\
\text { angles }\end{array}$ & 7,12 \\
\hline E355K & $1063 \mathrm{G}>\mathrm{A}$ & Severe & 39 & Surface & $\begin{array}{l}\text { Loss of surface salt bridge with } \\
\text { Arg304, and of } \mathrm{H} \text { bonds with } \\
\text { Ser309, Glu310; charge switch }\end{array}$ & 8 \\
\hline S364R & $1092 \mathrm{C}>\mathrm{G}$ & NR & 1 & Buried & $\begin{array}{l}\text { Loss of } \mathrm{H} \text { bonds with Gln83, His } 84 \text {; } \\
\text { marked steric clash in buried posi- } \\
\text { tion }\end{array}$ & 3 \\
\hline E369K & $1105 \mathrm{G}>\mathrm{A}$ & Severe & 6 & Surface & $\begin{array}{l}\text { Loss of } \mathrm{H} \text { bond with Gln } 400 \text {; } \\
\text { charge switch close to active site }\end{array}$ & $6,9,16,26,28$ \\
\hline Y374H & $1120 \mathrm{~T}>\mathrm{C}$ & Severe & 2 & Buried & Unsatisfied $\mathrm{H}$ bonding; charge & 8 \\
\hline $\mathrm{R} 377 \mathrm{C}$ & $1129 \mathrm{C}>\mathrm{T}$ & Severe & 0 & Buried & $\begin{array}{l}\text { Loss of buried salt bridge with } \\
\text { Asp477, and of } \mathrm{H} \text { bonds with } \\
\text { Ser366, Met376; possible interfer- } \\
\text { ence with disulfide bridge forma- } \\
\text { tion }\end{array}$ & 6,13 \\
\hline $\mathrm{R} 377 \mathrm{H}$ & $1130 \mathrm{G}>\mathrm{A}$ & Severe & 0 & Buried & $\begin{array}{l}\text { Loss of buried salt bridge with } \\
\text { Asp477, and of H bonds with } \\
\text { Ser366, Met376 }\end{array}$ & $3,4,11,26$ \\
\hline
\end{tabular}




\begin{tabular}{|c|c|c|c|c|c|c|}
\hline Protein & Codon & Phenotype & NASA $(\%)$ & Type & Effect of mutation on structure & Reference \\
\hline R377L & $1130 \mathrm{G}>\mathrm{T}$ & NR & 0 & Buried & $\begin{array}{l}\text { Loss of buried salt bridge with } \\
\text { Asp } 477 \text {, and of } \mathrm{H} \text { bonds with } \\
\text { Ser } 366, \operatorname{Met} 376\end{array}$ & 28 \\
\hline Q380R & $1139 A>G$ & Severe & 2 & Buried & $\begin{array}{l}\text { Gain of charge in buried position } \\
\text { close to surface; steric clash may af- } \\
\text { fect } \mathrm{H} \text { bond with Arg } 382\end{array}$ & 4,26 \\
\hline L386R & $1157 \mathrm{~T}>\mathrm{G}$ & Severe & 0 & Buried & $\begin{array}{l}\text { Introduction of charge and steric } \\
\text { clash in buried position; disruption } \\
\text { of hydrophobic interactions }\end{array}$ & 7,12 \\
\hline V387M & $1159 \mathrm{G}>\mathrm{A}$ & NR & 2 & Buried & $\begin{array}{l}\text { Introduction of bulky residue in } \\
\text { buried position }\end{array}$ & 22 \\
\hline N389S & $1166 \mathrm{~A}>\mathrm{G}$ & NR & 0 & Buried & $\begin{array}{l}\text { Loss of buried } \mathrm{H} \text { bonds with } \\
\text { Ala } 434 \text {, Glu437 }\end{array}$ & 28 \\
\hline N389K & $1167 \mathrm{C}>\mathrm{A}$ & NR & 0 & Buried & $\begin{array}{l}\text { Loss of buried } \mathrm{H} \text { bonds with } \\
\text { Ala434, Glu437; steric clash }\end{array}$ & 3,26 \\
\hline L411R & $1232 \mathrm{~T}>\mathrm{G}$ & NR & 1 & Buried & $\begin{array}{l}\text { Introduction of charge in buried } \\
\text { position; clash; disruption of hy- } \\
\text { drophobic interactions }\end{array}$ & 26 \\
\hline $\mathrm{T} 415 \mathrm{P}$ & $1243 \mathrm{~A}>\mathrm{C}$ & NR & 34 & Surface & $\begin{array}{l}\text { Loss of } \mathrm{H} \text { bond with Leu } 411 \text {; steric } \\
\text { clash with Leu } 411 ; \text { kink in helix } \\
\alpha 11 \text { close to glycosylation site }\end{array}$ & 28 \\
\hline $\mathrm{T} 421 \mathrm{R}$ & $1262 \mathrm{C}>\mathrm{G}$ & Mild & 16 & Surface & $\begin{array}{l}\text { Loss of } \mathrm{H} \text { bond with Trp423; sol- } \\
\text { vent exposure accommodates bulky } \\
\text { sc }\end{array}$ & 26 \\
\hline R433W & $1297 \mathrm{C}>\mathrm{T}$ & Severe & 6 & Buried & $\begin{array}{l}\text { Loss of buried } \mathrm{H} \text { bonds with } \\
\text { Asn } 284 \text {, Tyr } 430 \text { and of charge; } \\
\text { steric clash }\end{array}$ & $8,11,12,18,28$ \\
\hline R433Q & $1298 \mathrm{G}>\mathrm{A}$ & Severe & 6 & Buried & $\begin{array}{l}\text { Loss of buried } \mathrm{H} \text { bonds with } \\
\text { Asn } 284 \text {, Tyr } 430 \text { and of charge; } \\
\text { destabilizes packing }\end{array}$ & $12,16,26$ \\
\hline D444G & $1331 \mathrm{~A}>\mathrm{G}$ & Mild & 13 & Surface & $\begin{array}{l}\text { Loss of surface } \mathrm{H} \text { bonds with } \\
\text { Thr } 448 \text {, Gln } 449\end{array}$ & 14 \\
\hline E447K & $1339 \mathrm{G}>\mathrm{A}$ & Severe & 8 & Surface & $\begin{array}{l}\text { Switch of charge in partly buried } \\
\text { position }\end{array}$ & 2,12 \\
\hline Q472H & $1416 \mathrm{G}>\mathrm{C}$ & NR & 6 & Surface & Loss of $\mathrm{H}$ bond with Asp477 & 28 \\
\hline V486F & $1456 \mathrm{G}>\mathrm{T}$ & Severe & 37 & Dimer interface & Disruption of dimer interface & 8 \\
\hline
\end{tabular}

References: 1) Scott et al., 1995; 2) Blanch et al., 1997; 3) Bunge et al., 1997; 4) Weber et al., 1997; 5) Weber et al., 1998; 6) Di Natale et al., 1998; 7) Montfort et al., 1998; 8) Beesley et al., 2000; 9) Esposito et al., 2000; 11) Yogalingam and Hopwood, 2001; 12) Chabás et al., 2001; 13) Lee-Chen et al., 2002; 14) Miyazaki et al., 2002; 15) Emre et al., 2002; 16) Di Natale et al., 2003; 17) Van Hove et al., 2003; 18) Muschol et al., 2004; 19) Bekri et al., 2005; 20) Gabrielli et al., 2005; 21) Fiorentino et al., 2006; 22) Di Natale et al., 2006; 23) Meyer et al., 2008; 24) Zhang and Huiping, 2008; 25) Piotrowska et al., 2009; 26) Valstar et al., 2010; 27) Muschol et al., 2011; 28) Pollard et al., 2013. Some of the references were located from a partial listing at the Human Gene Mutation Database, http://www.hgmd.org/. Abbreviations: H bond(ing): hydrogen bond(ing); Interm.: intermediate; NASA: normalized accessible surface area per atom per residue (as percent of maximum value for any non-terminal residue in the atomic model of SGSH); NR: Not reported; sc: side chain. Table adapted from Sidhu et al. (2014). 
Although approximately a quarter of the missense mutations map to the enzyme surface, half of the 12 mild-phenotype ones map to the surface. In addition, in the case of two others, E292K and S298P, a buried position of the wild-type residue coincides with buried waters next to the side chain, possibly providing the tightly packed enzyme with extra space to accommodate the mutated side chain, presumably by displacing the waters.

The structure clearly identifies the $\mathrm{Ca}^{2+}$-binding residues. Mutations affecting these residues predictably lead to a severe phenotype, as in the case of D32G and D273N. However, in the mutation D32E (Asp32Glu), the $\mathrm{Ca}^{2+}$-binding is apparently conserved due to the conservative nature of the mutation.

\subsubsection{Molecular basis of mutations with a geographical distribution bias}

Some of the mutations occur more frequently in particular regions of the world. These include S66W (Ser66Trp), R74C (Arg74Cys) and R245H (Arg245His). Of these, Ser66Trp is especially common in Italy (33\% of disease alleles) (Di Natale et al., 1998). Ser66 is a buried residue. Its replacement by the bulky aromatic tryptophan would disrupt packing of the wild-type structure of the enzyme. Additionally, this disruption occurs in a 5-residue loop that is followed by the helix $\alpha 2$, which houses active site residues including the crucial FGly 70 .

Arg74Cys is particularly frequent in Poland (56\%), Finland and Estonia (50\%), and Germany (21\%) (Bunge et al., 1997). Arg74 is a buried active site residue that lies in one of the sulfatase signature sequences (residues 70-80). It forms hydrogen bonds or salt bridges with several residues that coordinate $\mathrm{Ca}^{2+}$ in the active site, including FGly70, Asp31 and Asp273 (Fig. 3.27). It is strictly conserved in all 6 O-sulfatases for which the structures are known. The conservation includes $\mathrm{BcPMH}$, in which only 5 of 10 important active site residues are conserved. Mutations affecting Arg74 would be predicted to affect the catalytic activity and likely the stability of sulfamidase. Accordingly, a severe phenotype has been reported for both missense mutations at this site, Arg74Cys (Bunge et al., 1997; Weber et al., 1997; Di Natale et al., 1998; Beesley et al., 2000; Esposito et al., 2000; Emre et al., 2002; Muschol et al., 2004; Meyer et al., 2008; Piotrowska et al., 2009; Valstar et al., 2010; Muschol et al., 2011; Pollard et al., 2013) and Arg74His (Bunge et al., 1997; Chabás et al., 2001).

The mutation $\operatorname{Arg} 245 \mathrm{His}(\mathrm{R} 245 \mathrm{H})$ is observed with a high relative prevalence in the Netherlands, where it occurs with an allele frequency of $44.9 \%$ of all alleles identified in MPS IIIA patients (Valstar et al., 2010). It is also relatively frequent in Germany (35\%), US (21.5\%) and the UK (20\%) (Weber et al., 1997; Beesley et al., 2000; Pollard et al., 2013). The side chain of Arg245 is buried in the middle of the 30-residue long helix $\alpha 7$, forming a salt bridge with Asp179 and hydrogen bonds with it and Cys194. In this position, a histidine would be a poor substitution 

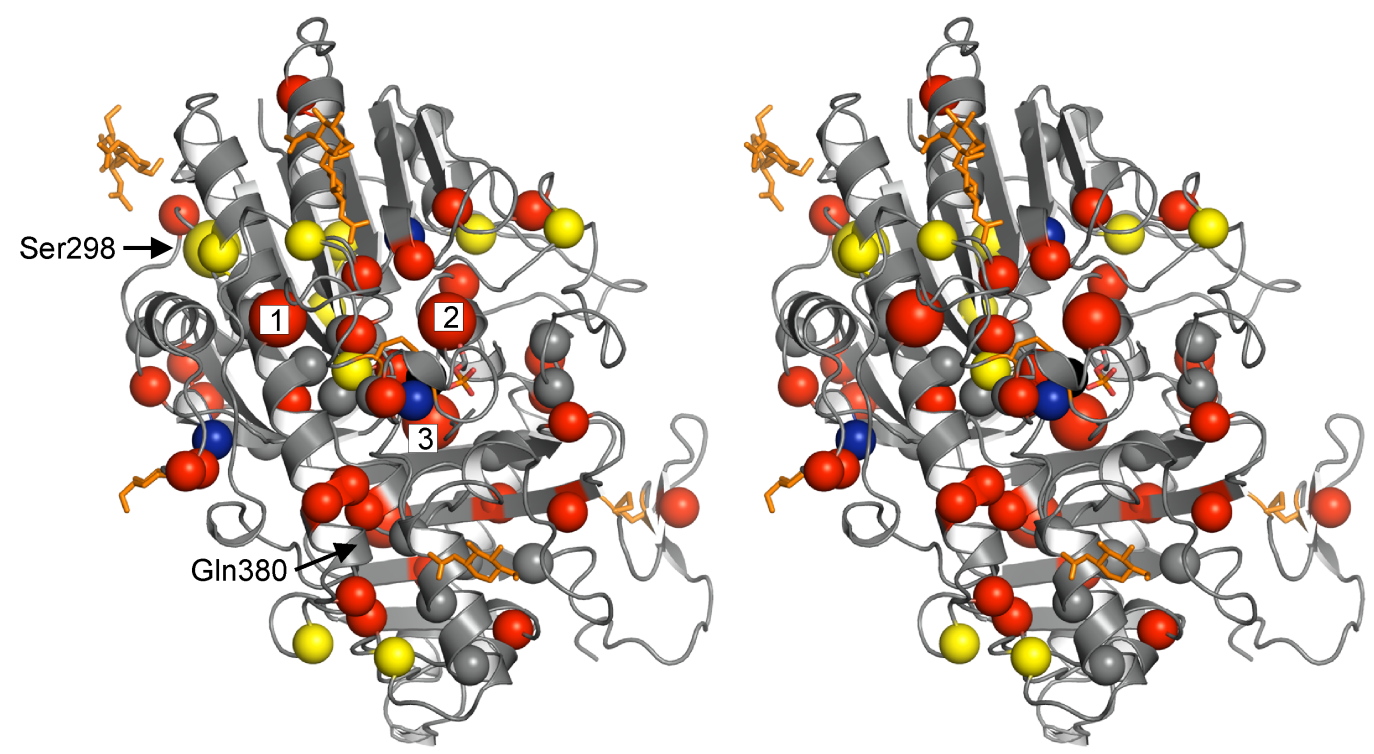

Figure 3.26: Stereo view of disease-causing missense mutations mapped onto the enzyme 3-dimensional structure. The $\mathrm{C} \alpha$ atoms of the mutated residue is shown as a ball. The color coding is as follows: Severe phenotype is denoted by a red ball; intermediate-severity phenotype by a blue ball; mild or attenuated by a yellow one. Where the mutation was reported without reporting a phenotype, a gray ball is shown. FGly70 is displayed as a stick model (with an almost hidden $\mathrm{Ca}^{2+}$ ion as a black ball) for orientation, which is in the front view as defined above. Most mutation sites display a severe phenotype. For two different phenotypes at the same site, the most severe phenotype is typically displayed. Mild mutations (yellow) appear to show a tendency to lie close to the periphery of the molecule. More common mutations are shown as a ball each of a larger size. These are at Ser298, Arg245 (labeled as 1), Arg74 (2), Ser66 (3) and Gln380. Figure published in Sidhu et al. (2014); copyright IUCr; used with permission. 


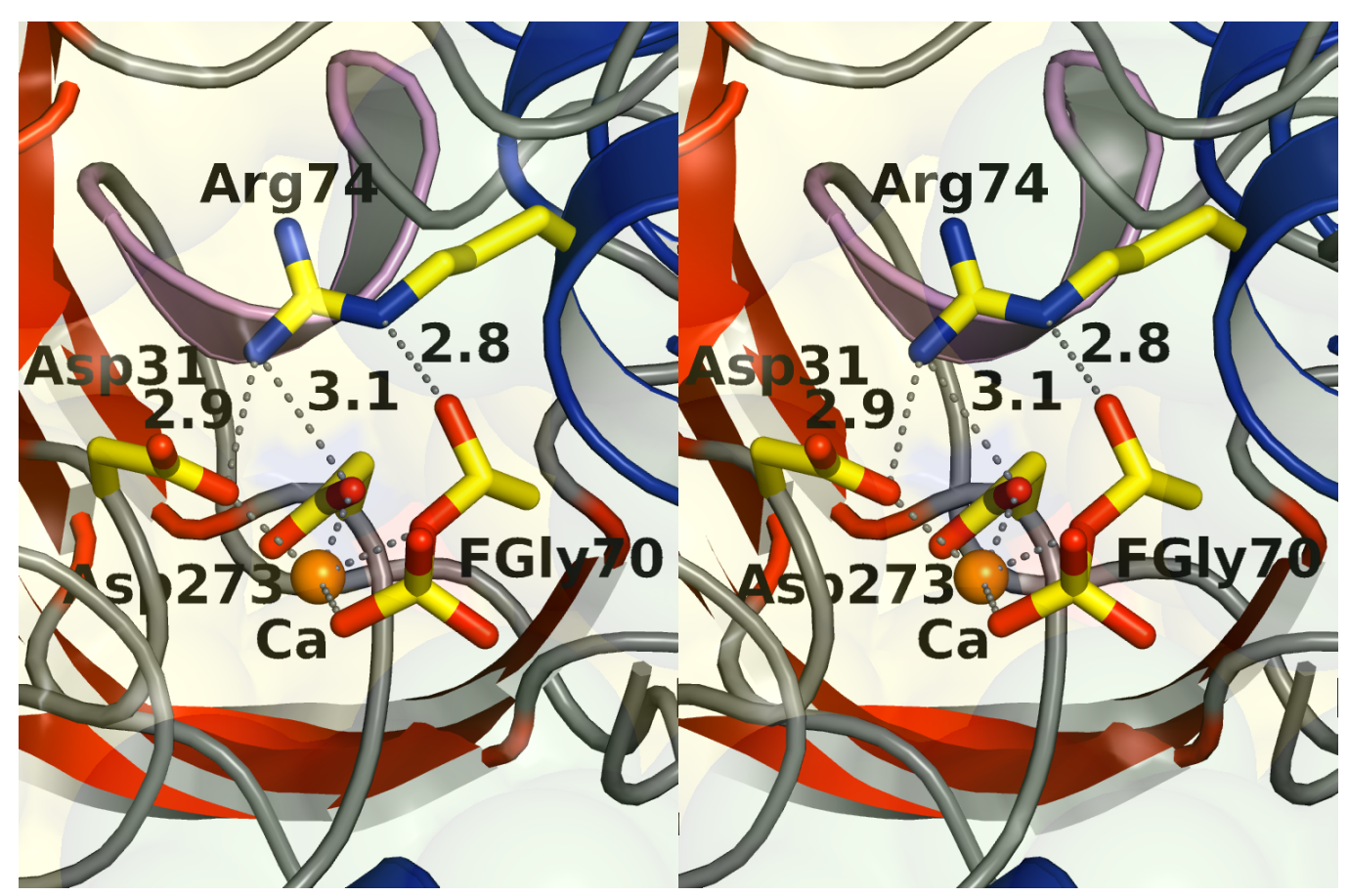

Figure 3.27: Stereo view of the structural environment of $\operatorname{Arg} 74$, the site of a common mutation in MPS IIIA. It forms salt bridges and/or hydrogen bonds with active site residues Asp31, Asp273, and FGly70, all of which bind $\mathrm{Ca}^{2+}$ (labeled $\mathrm{Ca}$ ). Interactions and distances are indicated by dashes and numbers in $\AA$, respectively. Residues are shown as sticks, with carbons in yellow. Colors of secondary structure elements follow the standard colors used in the Homodimer section above. 


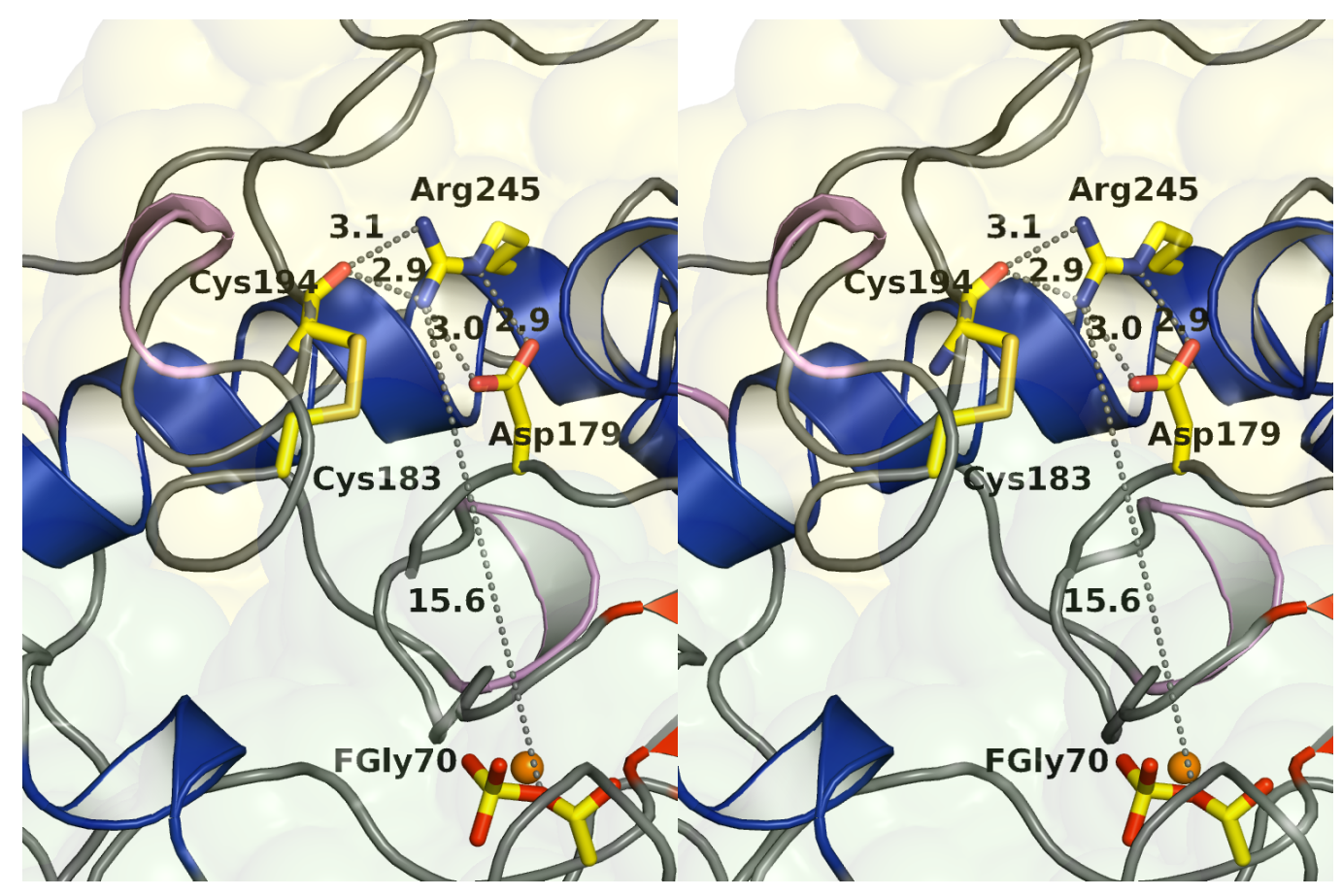

Figure 3.28: Stereo view of the structural environment of $\operatorname{Arg} 245$, the site of a common mutation in MPS IIIA. It is a buried charged residue that forms a salt bridge and/or hydrogen bonds with Asp179 and Cys194, and lies about $15 \AA$ from the active site FGly70. The rest of the representation details are as in Fig. 3.27. A histidine would be disruptive here as it would be too short and too bulky at the base close to the main chain.

for arginine as it would be too bulky close to the main chain to be properly buried and too short to form the appropriate interactions. The switch would thus be predicted to destabilize local structure and packing. Consistent with this view, enzyme activity has been reported to be absent in this mutation (Perkins et al., 1999).

\subsubsection{Mutations affecting the dimer interface}

The structure identifies residues forming the dimer interface. All 10 chains in the two present structures of the enzyme occur as parts of 5 dimers. Active human sulfamidase has been isolated as a dimer (Freeman and Hopwood, 1986; Paschke and Kresse, 1979). Only 1 missense mutation directly affects a residue at the dimer interface, Val486Phe, a mutation causing a severe phenotype apparently due to the bulky replacement at the interface. However, it is likely that other mutations, especially so those affecting neighboring residues, also destabilize the 
dimer interface indirectly.

As pointed out above, nonsense mutations have been linked as a rule to a severe phenotype, suggesting its imprtant functional role in sulfamidase. The structure offers a possible explanation for this observation. Many residues forming the dimer interface lie not only in Domain 2 but also in close proximity to the C-terminus (residue 502) of the enzyme: Leu487, Glu488, Pro497, Leu498 and Asn500. Thus, nonsense mutations may be predicted as a rule to destabilize the dimer interface. Since the second subunit lies close to the entry into the active site pocket, it appears plausible that the destabilization of the dimer interface has a disruptive effect on substrate binding.

\subsubsection{Limitations and prospects}

The structure of the wild-type enzyme provides a rational starting point to understand the molecular basis of disease in MPS IIIA, with limitations, some of which are pointed out as unclear aspects in Table 3.3. Another example is that of the missense mutation S106R (Ser106Arg), which occurs at a buried position. Based on the structure, it might be predicted to be associated with a severe disease phenotype. However, the reported phenotype is a mild one. It appears plausible that the enzyme is able to accommodate the larger side chain through local structural changes in a loop that is partly solvent-exposed. Mutations may cause secondary structural changes to the wild-type structure. Often this would negatively affect function. However, a degree of structural flexibility may allow amelioration of the destabilization caused by a mutation.

For optimal molecular interpretation of the likely consequences of mutations, the present structural study of the wild-type enzyme needs to be complemented by other information, including clinical phenotype information, structural studies of the enzyme with mutations incorporated, and an investigation of dynamics. It is, of course, not possible to fully predict the function of the wild-type enzyme, much less the effects of mutations, based on the crystal structure of the wild-type enzyme alone. Functional interpretations based on the present structure alone need to be made with appropriate care. This is especially so due to the association of many of the known mutations of the enzyme with a clinically manifested human disorder.

A naturally-occurring mouse model of MPS IIIA with the D31N mutation has been described (Gliddon et al., 2004). The study also indicated that human MPS IIIA fibroblasts displaying lysosomal storage phenotype could endocytose recombinant murine sulfamidase through the mannose-6-phosphate receptor, resulting in a correction of the phenotype. Murine sulfamidase shares an $88 \%$ amino acid sequence identity with human sulfamidase. The present structure should therefore help in developing a better homology model for the murine enzyme, which may 
then be used for further research into treatment options for MPS IIIA.

Apart from this, structural work foreseen for future studies with sulfamidase includes testing the role of Arg282 in substrate binding via mutagenesis experiments, including an Arg282Lys mutant. In addition, it would be possible to explore the possibility of solving structures of sulfamidase molecules containing the most common mutations. In addition the enzymatic mechanism could be explored further by solving crystal structures with combinations of substrates, inhibitors, and other divalent metal ions.

It is speculated that the structure should be helpful in designing and testing modifications to the enzyme, e.g. to test improved transport across the blood-brain barrier or its cellular uptake and delivery to the lysosomes, as also for computational binding and other ligand design studies. Thus, it seems that both the $\mathrm{N}$ - and C-termini of sulfamidase are amenable to chimeric modifications that may be of benefit to explore (Sly and Vogler, 2013). Certainly much more research is still needed. The solution of the structure of sulfamidase represents only one step in an effort to better understand the molecular basis of disease in this disorder.

\subsection{Conclusions}

The crystal structure of two crystal forms of mature glycosylated recombinant human sulfamidase was solved and refined against data to $2.00 \AA$ and $2.40 \AA$. This is the first three-dimensional structure of a sulfamidase that has been described in the literature. To the best of my knowledge, it also appears to be the first structure of a hydrolase that is recognized to catalyze the cleavage a sulfate from an S-N linkage in a substrate, which in the case of sulfamidase are the glycosaminoglycans heparin and heparan sulfate.

The structure was solved using molecular replacement using search model fragments derived from a distant bacterial O-sulfatase homologue. The bacterial sulfatase shared a relatively low, approximately $22 \%$ structure-based sequence identity with the sulfamidase sequence, and a relatively high backbone $\mathrm{C}_{\alpha}$ RMSD of $2.2 \AA$ over $71 \%$ of the residues in the final sulfamidase model. However, a rotation-translation solution was obtained using molecular replacement as implemented in ARCIMBOLDO/PHASER. However, the electron density map derived from this solution was essentially uninterpretable initially. However, it was possible to derive an improved solution using the initial solution as a starting point and initiate model rebuilding, successfully refining and solving the sulfamidase structure.

The enzyme possesses a sulfatase fold. It occurs as a homodimer in both crystal forms, with 2 and 8 molecules in the asymmetric unit. The active site lies in a narrow pocket in a surface cleft and contains a formylglycine residue, a divalent metal ion binding site and a sulfate binding site. Nine of 10 important 
catalytic residues are conserved between sulfamidase and most of the five specific O-sulfatase homologues with known structures, suggesting a shared enzymatic mechanism, which is proposed. However, the pocket leading into the active site appears to be variable between sulfatases, apparently allowing different types of substrates to undergo a similar enzymatic reaction. 




\section{Appendix}

\section{Data quality indicators}

$\mathbf{R}_{\text {sym }}$, also known as $\mathbf{R}_{\text {int }}$ (as intensities are used rather than structure factor amplitudes), $\mathrm{R}_{\text {linear }}$ and, at times, $\mathrm{R}_{\text {merge }}$, is defined as

$$
R_{\text {sym }}=\frac{\sum_{\text {uniqueh }}\left(\sum_{i=1}^{N}|I-\bar{I}|\right)}{\sum_{\text {unique } \mathbf{h}}\left(\sum_{i=1}^{N} I\right)},
$$

where $N$ is the number of independent measurements of a reflection $\boldsymbol{h}$ measured more than once, $I$ is the intensity of the reflection and $\bar{I}$ is the mean intensity. Its use is superseded by the following indicators (Einspahr and Weiss, 2012) as it penalizes a greater redundancy in equivalents measured (Weiss and Hilgengeld, 1997) while the following ones do not.

Redundancy-independent merging $\mathrm{R}$-value, $\mathrm{R}_{\text {rim }}$ or $\mathrm{R}_{\text {meas }}$ (Diederichs and Karplus, 1997), is a measure of the precision of unmerged data. It is defined as

$$
R_{\text {rim }}=\frac{\sum_{\text {uniqueh }}\left(\left(\frac{N}{N-1}\right)^{\frac{1}{2}} \sum_{i=1}^{N}|I-\bar{I}|\right)}{\sum_{\text {uniqueh }}\left(\sum_{i=1}^{N} I\right)},
$$

where $N$ is the number of equivalents of a unique reflection $\boldsymbol{h}, I$ is the intensity of the reflection and $\bar{I}$ is the mean intensity.

$\mathrm{R}_{\text {pim }}$ is a measure of the precision of merged data and is defined as

$$
R_{\text {pim }}=\frac{\sum_{\text {uniqueh }}\left((N-1)^{\frac{-1}{2}} \sum_{i=1}^{N}|I-\bar{I}|\right)}{\sum_{\text {unique } \mathbf{h}}\left(\sum_{i=1}^{N} I\right)} .
$$

\section{Additional Software and Programming}

Apart from the software mentioned in the main text, additional software used in preparing the thesis included the following. Some calculations were performed and figures produced using PostScript and $\mathrm{C}++$. Some figures were processed (e.g. figure format conversion, cropping, joining, text addition) using ImageMagick, $<$ http://www.imagemagick.org $>$, and gimp, <www.gimp.org $>$. A few am- 
biguities in journal abbreviations were resolved using the American Chemical Society's CAS Source Index (CASSI) search tool, < cassi.cas.org/search.jsp $>$. This document was typeset using $\mathrm{ITT}_{\mathrm{E}} \mathrm{X}$. Computers used typically ran a Linux-based operating system. 


\section{Literature}

Anderson, B. M. \& Anderson, C. D. (1963). Effect of buffers on nicotinamide adenine dinucleotide hydrolysis. J. Biol. Chem. 238, 1475-1478.

Audette, G. F., Quail, J. W., Hayakawa, K., Bai, C., Chen, R. D. \& Delbaere, L. T. J. (1999). Crystallization and preliminary x-ray diffraction studies of monomeric isocitrate dehydrogenase from Corynebacterium glutamicum. Acta Crystallogr. D55, 1584-1585.

Bai, C., Fernandez, E., Yang, H. \& Chen, R. (1999). Purification and stabilization of a monomeric isocitrate dehydrogenase from Corynebacterium glutamicum. Protein Expr. Purif. 15, 344-348.

Banerjee, S., Nandyala, A., Podili, R., Katoch, V. M., Murthy, K. J. R. \& Hasnain, S. E. (2004). Mycobacterium tuberculosis (MTB) isocitrate dehydrogenases show strong B cell response and distinguish vaccinated controls from TB patients. Proc. Natl. Acad. Sci. USA 101, 12652-12657.

Barrera, C. R. \& Jurtshuk, P. (1970). Characterization of the highly active isocitrate NADP dehydrogenase EC-1.1.1.42 of Azotobacter vinelandii. Biochim. Biophys. Acta 220, 416-429.

Beesley, C. E., Young E. P., Vellodi, A., \& Winchester, B. G. (2000). Mutational analysis of Sanfilippo syndrome type A (MPS IIIA): identification of 13 novel mutations. J. Med. Genet. 37, 704-707.

Bekri, S., Armana, G., De Ricaud, D., Osenda, M., Maire, I., Van Obberghen, E. \& Froissart, R. (2005). Early diagnosis of mucopolysaccharidosis III A with a nonsense mutation and two de novo missense mutations in SGSH gene. J. Inherit. Metab. Dis. 28, 601-602.

Berman, H. M., Westbrook, J., Feng, Z., Gilliland, G., Bhat, T. N., Weissig. H., Shindyalov, I. N. \& Bourne, P. E. (2000). The Protein Data Bank. Nucleic Acids Res. 28, 235-242.

Bernfield, M., Götte, M., Park, P. W., Reizes, O., Fitzgerald, M. L., Lincecum, J., Zako, M. (1999). Functions of cell surface heparan sulfate proteoglycans. Annu. Rev. Biochem. 68, 729-77.

Bielicki, J., Fuller, M., Guo, X.-H., Morris, C. P., Hopewood, J. J. \& Anson, 
D. S. (1995). Expression, purification and characterization of recombinant human N-acetylgalactosamine-6-sulphatase. Biochem. J. 311, 333-339.

Blanch, L., Weber, B., Guo, X.-H., Scott, H. S. \& Hopwood, J. J. (1997). Molecular defects in Sanfilippo syndrome type A. Hum. Mol. Genet. 6, 787-791.

Bolduc, J. M., Dyer, D. H., Scott, W. G., Singer, P., Sweet, R. M., Koshland, D. E. Jr \& Stoddard, B. L. (1995). Mutagenesis and Laue structures of enzyme intermediates: isocitrate dehydrogenase. Science 268, 1312-1318.

Boltes, I., Czapinska, H., Kahnert, A., von Bülow, R., Dierks, T., Schmidt, B., von Figura, K., Kertesz, M. A. \& Usón, I. (2001). 1.3 angstrom structure of arylsulfatase from Pseudomonas aeruginosa establishes the catalytic mechanism of sulfate ester cleavage in the sulfatase family. Structure 9, 483-491.

Bond, C. S., Clements, P. R., Ashby, S. J., Collyer, C. A., Harrop, S. J., Hopwood, J. J. \& Guss, J. M. (1997). Structure of a human lysosomal sulfatase. Structure 5, 277-289.

Bragg, W.H. (1914). X-rays and crystalline structure. Science 40, 795-802.

Brünger, A. T. (1992). Free R value: a novel statistical quantity for assessing the accuracy of crystal structures. Nature (London, $U$. K.) 355, 472-475.

Bunge, S., Ince, H., Steglich, C., Kleijer, W. J., Beck, M., Zaremba, J., van Diggelen, O. P., Weber, B., Hopwood, J. J. \& Gal, A. (1997). Identification of 16 sulfamidase gene mutations including the common R74C in patients with mucopolysaccharidosis type IIIA (Sanfilippo A). Hum. Mutat. 10, 479-485.

Burla, M. C., Carrozzini, B., Cascarano, G. L., Giacovazzo, C. \& Polidori, G. (2000). Solving crystal structures in $P 1$ : an automated procedure for finding an allowed origin in the correct space group. J. Appl. Crystallogr. 33, 307-311.

Capila, I. \& Linhardt, R.J. (2002). Heparin-Protein interactions. Angew. Chem. Int. Ed. 41, 390-412.

Chabás, A., Montfort, M., Martínez-Campos, M., Díaz, A., Coll, M. J., Grinberg, D. \& Vilageliu, L. (2001). Mutation and haplotype analyses in 26 Spanish Sanfilippo syndrome type A patients: possible single origin for 1091delC mutation. Am. J. Med. Genet. 100, 223-228. 
Chen, R. \& Gadal, P. (1990). Structure, functions and regulation of NAD and NADP dependent isocitrate dehydrogenases in higher plants and in other organisms. Plant Physiol. Biochem. 28, 411-427.

Chen, R., Greer, A. \& Dean, A. M. (1995). A highly active decarboxylating dehydrogenase with rationally inverted coenzyme specificity. Proc. Natl. Acad. Sci. USA 92, 11666-11670.

Chen, R., Greer, A. F. \& Dean, A. M. (1997a). Structural constraints in protein engineering: the coenzyme specificity of Escherichia coli isocitrate dehydrogenase. Eur. J. Biochem. 250, 578-582.

Chen, Y., Maguire, T., Hileman, R. E., Fromm, J. R., Esko, J. D., Linhardt, R. J. \& Marks, R. M. (1997b). Dengue virus infectivity depends on envelope protein binding to target cell heparan sulfate. Nature Medicine 3, 866-871.

Chen, R. \& Yang, H. (2000). A highly specific monomeric isocitrate dehydrogenase from Corynebacterium glutamicum. Arch. Biochem. Biophys. 383, 238-245.

Chen, V. B., Arendall, W. B., Headd, J. J., Keedy, D. A., Immormino, R. M., Kapral, G. J., Murray, L. W., Richardson, J. S. \& Richardson, D. C. (2010). Molprobity: all-atom structure validation for macromolecular crystallography. Acta Crystallogr. D66, 12-21.

Chothia, C. \& Lesk, A. M. (1986). The relation between the divergence of sequence and structure in proteins. EMBO J. 5, 823-826.

Chung, A. E. \& Franzen, J. S. (1969). Oxidized triphosphopyridine nucleotide specific isocitrate dehydrogenase from Azotobacter vinelandii. Isolation and characterization. Biochemistry 8, 3175-3184.

Clegg, W. (2006a). Fourier syntheses. In Clegg, W. (Ed.). Crystal Structure analysis: Principles and practice. 2nd edition. Oxford: International Union of Crystallography/Oxford University Press, pp. 103-115.

Clegg, W. (2006b). Patterson syntheses for structure determination. In Clegg, W. (Ed.). Crystal Structure analysis: Principles and practice. 2nd edition. Oxford: International Union of Crystallography/Oxford University Press.

Collaborative Computational Project, Number 4 (1994). The CCP4 suite: pro- 
grams for protein crystallography. Acta Crystallogr. D50, 760-763.

Cowtan, K. (1994). DM: An automated procedure for phase determination by density modification. Jnt. CCP4/ESF-EACBM Newsl. Protein Crystallogr., 31, 34-38.

Cowtan, K. (2006). The Buccaneer software for automated model building. 1. Tracing protein chains. Acta Crystallogr. D62, 1002-1011.

Dang, L., White, D. W., Gross, S., Bennett, B. D., Bittinger, M. A., Driggers, E. M., Fantin, V. R., Jang, H. G., Jin, S., Keenan, M. C., Marks, K. M., Prins, R. M., Ward, P. S., Yen, K. E., Liau, L. M., Rabinowitz, J. D., Cantley, L. C., Thompson, C. B., Vander Heiden, M. G. \& Su, S. M. (2009). Cancer-associated IDH1 mutations produce 2-hydroxyglutarate. Nature (London, U. K.) 462, 739-744.

Daniele, A. \& Di Natale, P. (2001). Heparan N-sulfatase: cysteine 70 plays a role in the enzyme catalysis and processing. FEBS Lett. 505, 445-448.

Davis, I. W., Leaver-Fay, A., Chen, V. B., Block, J. N., Kapral, G. J., Wang, X., Murray, L. W., Arendall, W. B. III, Snoeyink, J., Richardson, J. S. \& Richardson, D. C. (2007). MolProbity: all-atom contacts and structure validation for proteins and nucleic acids. Nucleic Acids Res. 35, W375-W383.

Dean, A. M. \& Koshland, D. E. Jr (1993). Kinetic mechanism of Escherichiacoli isocitrate dehydrogenase. Biochemistry 32, 9302-9309.

Dean, A. M. \& Dvorak, L. (1995). The role of glutamate-87 in the kinetic mechanism of Thermus-thermophilus isopropylmalate dehydrogenase. Protein Sci. 4, 2156-2167.

Dean, A. M., Shiau, A. K. \& Koshland, D. E. Jr (1996). Determinants of performance in the isocitrate dehydrogenase of Escherichia coli. Protein Sci. 5, 341-347.

Dean, A. M. \& Golding, G. B. (1997). Protein engineering reveals ancient adaptive replacements in isocitrate dehydrogenase. Proc. Natl. Acad. Sci. USA 94, 3104-3109.

DeLano, W. L. (2002). PyMOL. http://www.pymol.org.

Diederichs, K. \& Karplus, P.A. (1997). Improved $R$-factors for diffraction data 
analysis in macromolecular crystallography. Nat. Struct. Biol. 4, 269-275.

Dierks, T., Dickmanns, A., Preusser-Kunze, A., Schmidt, B., Mariappan, M., von Figura, K., Ficner, R. \& Rudolph, M. G. (2005). Molecular basis for multiple sulfatase deficiency and mechanism for formylglycine generation of the human formylglycine-generating enzyme. Cell 121, 541-552.

DiMaio, F., Terwilliger, T. C., Read, R. J., Wlodawer, A., Oberdorfer, G., Wagner, U., Valkov, E., Alon, A., Fass, D., Axelrod, H. L., Das, D., Vorobiev, S. M., Iwaï, H., Pokkuluri, P. R. \& Baker, D. (2011). Improved molecular replacement by density-and energy-guided protein structure optimization. Nature (London, U. K.), 473, 540-543.

Di Natale, P., Balzano, N., Esposito, S. \& Villani, G. R. (1998). Identification of molecular defects in Italian Sanfilippo A patients including 13 novel mutations. Hum. Mutat. 11, 313-320.

Di Natale, P., Vanacore, B., Daniele, A. \& Esposito, S. (2001). Heparan NSulfatase: In vitro mutagenesis of potential N-glycosylation sites. Biochem. Biophys. Res. Commun.280, 1251-1257.

Di Natale, P., Villani, G. R., Di Domenico, C., Daniele, A., Dionisi Vici, C. \& Bartuli, A. (2003). Analysis of Sanfilippo A gene mutations in a large pedigree. Clin. Genet. 63, 314-318.

Di Natale, P., Pontarelli, G., Villani, G. R. D. \& Di Domenico, C. (2006). Gene symbol: SGSH. Disease: Sanfilippo type A syndrome, mucopolysaccharidosis IIIA. Hum. Genet. 119, 679.

Eikmanns, B. J., Rittmann, D. \& Sahm, H. (1995). Cloning, sequence analysis, expression, and inactivation of the Corynebacterium-glutamicum icd gene encoding isocitrate dehydrogenase and biochemical characterization of the enzyme. J. Bacteriol. 177, 774-782.

Einspahr, H. M., \& Weiss, M. S. (2012). Quality indicators in macromolecular crystallography: definitions and applications. Int. Tables Crystallogr. F, 64-74.

Emre, S., Terzioglu, M., Tokatli, A., Coskun, T., Ozalp, I., Weber, B. \& Hopwood, J. J. (2002). Sanfilippo syndrome in Turkey: Identification of novel mutations in subtypes A and B. Hum. Mutat. 19, 184-185. 
Emsley, P., Lohkamp, B., Scott, W. G. \& Cowtan, K. (2010). Features and development of Coot. Acta Crystallogr. D66, 486-501.

Esposito, S., Balzano, N., Daniele, A., Villani, G. R. D., Perkins, K., Weber, B., Hopwood, J. J. \& Di Natale, P. (2000). Heparan N-sulfatase gene: two novel mutations and transient expression of 15 defects. Biochim. Biophys. Acta 1501, $1-11$.

Evans, P. \& McCoy, A. (2008). An introduction to molecular replacement. Acta Crystallogr. D64, 1-10.

Fiorentino, F., Biricik, A., Nuccitelli, A., De Palma, R., Kahraman, S., Iacobelli, M., Trengia, V., Caserta, D., Bonu, M. A., Borini, A. \& Baldi, M. (2006). Strategies and clinical outcome of 250 cycles of preimplantation genetic diagnosis for single gene disorders. Hum. Reprod. 21, 670-684.

Fokine, A., Capitani, G., Grütter, M. G. \& Urzhumtsev, A. (2003). Bulksolvent correction for fast translation search in molecular replacement: service programs for AMoRe and CNS. J. Appl. Crystallogr. 36, 352-355.

Freeman, C. \& Hopwood, J. J. (1986). Human liver sulphamate sulphohydrolase. Determinations of native protein and subunit Mr values and influence of substrate agylcone structure on catalytic properties. Biochem. J. 234, 83-92.

Fromm, J. R., Hileman, R. E., Caldwell, E. E., Weiler, J. M. \& Linhardt, R. J. (1995). Differences in the interaction of heparin with arginine and lysine and the importance of these basic amino acids in the binding of heparin to acidic fibroblast growth factor. Arch. Biochem. Biophys. 323, 279-287.

Fukunaga, N., Imagawa, S., Sahara, T., Ishii, A. \& Suzuki, M. (1992). Purification and characterization of monomeric isocitrate dehydrogenase with NADP+specificity from Vibrio parahaemolyticus Y-4. J. Biochem. 112, 849-855.

Gabrielli, O., Coppa, G. V., Bruni, S., Villani, G. R., Pontarelli, G. \& Di Natale, P. (2005). An adult Sanfilippo type A patient with homozygous mutation R206P in the sulfamidase gene. Am. J. Med. Genet. A 133, 85-89.

Garnak, M. \& Reeves, H. C. (1979). Phosphorylation of isocitrate dehydrogenase of Escherichia coli. Science 203, 1111-1112.

Gerstein, M., Lesk, A. M. \& Chothia, C. (1994). Structural mechanisms for 
domain movements in proteins. Biochemistry 33, 6739-6749.

Gerstmeir, R., Wendisch, V. F., Schnicke, S., Ruan, H., Farwick, M., Reinscheid, D. \& Eikmanns, B. J. (2003). Acetate metabolism and its regulation in Corynebacterium glutamicum. J. Biotechnol. 104, 99-122.

Ghosh, D. (2007). Human sulfatases: A structural perspective to catalysis. Cell. Mol. Life Sci. 64, 2013-2022.

Giugliani, R. (2012). Mucopolysaccharidoses: From understanding to treatment, a century of discoveries. Genet. Mol. Biol. 35, 924-931.

Gliddon, B. L., Yogalingam, G. \& Hopwood, J. J. (2004). Purification and characterization of recombinant murine sulfamidase. Mol. Genet. Metab. 83, 239-245.

Gonçalves, S., Miller, S. P., Carrondo, M. A., Dean, A. M. \& Matias, P.M. (2012). Induced fit and the catalytic mechanism of isocitrade dehydrogenase. Biochemistry 51, 7098-7115.

Goujon, M., McWilliam, H., Li, W., Valentin, F., Squizzato, S., Paern, J. \& Lopez, R. (2010). A new bioinformatics analysis tools framework at EMBL-EBI. Nucleic Acids Res. 38, W695-W699.

Grune, T. (2008). mtz2sca and mtz2hkl: facilitated transition from CCP4 to the SHELX program suite. J. Appl. Crystallogr. 41, 217-218.

Hanson, S. R., Best, M. D., \& Wong, C.-H. (2004). Sulfatases: Structure, mechanism, biological activity, inhibition, and synthetic utility. Angew. Chem. Int. Ed. 43, 5736-5763.

Harker, D. (1936). The application of the three-dimensional Patterson method and the crystal structures of proustite, $\mathrm{Ag}_{3} \mathrm{AsS}_{3}$, and pyrargyrite, $\mathrm{Ag}_{3} \mathrm{SbS}_{3} . J$. Chem. Phys. 4, 381-390.

Hauptman, H. A. (1991). The phase problem of x-ray crystallography. Rep. Prog. Phys. 54, 1427-1454.

Hayward, S. \& Berendsen, H. J. C. (1998). Systematic analysis of domain motions in proteins from conformational change: new results on citrate synthase and T4 lysozyme. Proteins: Struct., Funct., Genet. 30, 144-154. 
Helenius, A. \& Aebi, M. (2001). Intracellular functions of N-linked glycans. Science 291, 2364-2369.

Hernandez-Guzman, F. G., Higashiyama, T., Pangborn, W., Osawa, Y. \& Ghosh, D. (2003). Structure of human estrone sulfatase suggests functional roles of membrane association. J. Biol. Chem. 278, 22989-22997.

Hodel, A., Kim, S.-H. \& Brünger, A. T. (1992). Model bias in macromolecular crystal structures. Acta Crystallogr. A48, 851-858.

Hodel, A. E. \& Quiocho, F. A. (2012). Protein-ligand interactions. Int. Tables Crystallogr. F, 755-765.

Howlin, B., Butler, S. A., Moss, D. S., Harris, G. W. \& Driessen, H. P. C. (1993). TLSANL: TLS parameter-analysis program for segmented anisotropic refinement of macromolecular structures. J. Appl. Crystallogr. 26, 622-624.

Hunter, C. A. \& Sanders, J. K. M. (1990). The nature of $\pi-\pi$ interactions. $J$. Am. Chem. Soc. 112, 5525-5534.

Hurley, J. H., Thorsness, P. E., Ramalingam, V., Helmers, N. H., Koshland, D. E. Jr \& Stroud, R. M. (1989). Structure of a bacterial enzyme regulated by phosphorylation, isocitrate dehydrogenase. Proc. Natl. Acad. Sci. USA 86, 86358639.

Hurley, J. H., Dean, A. M., Sohl, J. L., Koshland, D. E. Jr \& Stroud, R. M. (1990). Regulation of an enzyme by phosphorylation at the active-site. Science 249, 1012-1016.

Hurley, J. H., Dean, A. M., Koshland, D. E. Jr \& Stroud, R. M. (1991). Catalytic mechanism of $\mathrm{NADP}^{+}$-dependent isocitrate dehydrogenase: implications from the structures of magnesium-isocitrate and $\mathrm{NADP}^{+}$complexes. Biochemistry 30, 8671-8678.

Hurley, J. H., Chen, R. \& Dean, A. M. (1996). Determinants of cofactor specificity in isocitrate dehydrogenase: structure of an engineered $\mathrm{NADP}^{+} \longrightarrow \mathrm{NAD}^{+}$ specificity-reversal mutant. Biochemistry 35, 5670-5678.

Imabayashi, F., Aich, S., Prasad, L. \& Delbaere, L. T. J. (2006). Substrate-free structure of a monomeric NADP isocitrate dehydrogenase: an open conformation 
phylogenetic relationship of isocitrate dehydrogenase. Proteins: Struct., Funct., Bioinf. 63, 100-112.

Imada, K., Tamura, T., Takenaka, R., Kobayashi, I., Namba, K. \& Inagaki, K. (2008). Structure and quantum chemical analysis of $\mathrm{NAD}^{+}$-dependent isocitrate dehydrogenase: hydride transfer and co-factor specificity. Proteins: Struct., Funct., Bioinf. 70, 63-71.

Iozzo, R. V. (1998). Matrix proteoglycans: From molecular design to cellular function. Annu. Rev. Biochem. 67, 609-652.

Ishii, A., Ochiai, T., Imagawa, S., Fukunaga, N., Sasaki, S., Minowa, O., Mizuno, Y. \& Shiokawa, H. (1987). Isozymes of isocitrate dehydrogenase from an obligately psychrophilic bacterium, Vibrio sp. strain ABE-1: purification, and modulation of activities by growth conditions. J. Biochem. 102, 1489-1498.

Jackson, R. L., Busch, S. J. \& Cardin, A. D. (1991). Glycosaminoglycans: Molecular properties, protein interactions, and role in physiological processes. Physiol. Rev. 71, 481-539.

Kabsch, W. (1976). A solution for the best rotation to relate two sets of vectors. Acta Crystallogr. A32, 922-923.

Kabsch, W. \& Sander, C. (1983). Dictionary of protein secondary structure: pattern recognition of hydrogen-bonded and geometrical features. Biopolymers $22,2577-2637$.

Kabsch, W. (2010). XDS. Acta Crystallogr. D66, 125-132.

Kadono, S., Sakurai, M., Moriyama, H., Sato, M., Hayashi, Y., Oshima, T. \& Tanaka, N. (1995). Ligand-induced changes in the conformation of 3-isopropylmalate dehydrogenase from Thermus thermophilus. J. Biochem. 118, 745-752.

Karageorgos, L. E., Guo, X.-H., Blanch, L., Weber, B., Anson, D. S., Scott, H. S. \& Hopwood, J. J. (1996). Structure and sequence of the human sulphamidase gene. DNA Res. 3, 269-271.

Karle, J. \& Hauptman, H. (1956). A theory of phase determination for the four types of non-centrosymmetric space groups $1 P 222,2 P 22,3 P_{1} 2,3 P_{2} 2$. Acta Crystallogr. 9, 635-651. 
Karlström, M., Stokke, R., Steen, I. H., Birkeland, N.-K. \& Ladenstein, R. (2005). Isocitrate dehydrogenase from the hyperthermophile Aeropyrum pernix: $\mathrm{x}$-ray structure analysis of a ternary enzyme-substrate complex and thermal stability. J. Mol. Biol. 345, 559-577.

Karplus, P. A. \& Diederichs, K. (2012). Linking crystallographic model and data quality. Science 336, 1030-1033.

Karpova, E. A., Voznyi, Y. V., Keulemans, J. L., Hoogeveen, A. T., Winchester, B., Tsvetkova, I. V. \& van Diggelen, O. P. (1996). A fluorimetric enzyme assay for the diagnosis of Sanfilippo disease type A (MPS IIIA). J. Inherit. Metab. Dis. 19, 278-285.

Klyne, W. \& Prelog, V. (1960). Description of steric relationships across single bonds. Experientia 16, 521-523.

Kornberg, H. L. \& Krebs, H. A. (1957). Synthesis of cell constituents from C2 units by a modified tricarboxylic acid cycle. Nature (London, U. K.) 179, 988-991.

Kornberg, H. L. (1966). Role and control of glyoxylate cycle in Escherichia coli: first Colworth medal lecture. Biochem. J. 99, 1-11.

Krebs, H. A. \& Johnson, W. A. (1937). The role of citric acid in intermediate metabolism in animal tissues. Enzymologia 4, 148-156.

Krissinel, E. \& Henrick, K. (2007). Inference of macromolecular assemblies from crystalline state. Pre-review of manuscript accepted in J. Mol. Biol.

Krivov, G. G., Shapovalov, M. V. \& Dunbrack, R. L. Jr (2009). Improved prediction of protein side-chain conformations with SCWRL4. Proteins: Struct., Funct., Bioinf. 77, 778-795.

LaPorte, D. C. \& Koshland, D. E. Jr (1982). A protein with kinase and phosphatase-activities involved in regulation of tricarboxylic-acid cycle. Nature (London, U. K.) 300, 458-460.

Lee, G. D. \& Van Etten, R. L. (1975). Purification and properties of a homogeneous aryl sulfatase A from rabbit liver. Arch. Biochem. Biophys. 166, 280-294.

Lee-Chen, G. J., Lin, S. P., Ko, M. H., Chuang, C. K., Chen, C. P., Lee, H. H., Cheng, S. C., Shen, C. H., Tseng, K. L. \& Li, C. L. (2002). Identification 
and characterization of mutations underlying Sanfilippo syndrome type A (mucopolysaccharidosis type IIIA). Clin. Genet. 61, 192-197.

Leyland, M. L. \& Kelly, D. J. (1991). Purification and characterization of a monomeric isocitrate dehydrogenase with dual coenzyme specificity from the photosynthetic bacterium Rhodomicrobium vannielii. Eur. J. Biochem. 202, 8593.

Linhardt, R. J. (2003). 2003 Claude S. Hudson Award address in carbohydrate chemistry. Heparin: Structure and activity. J. Med. Chem. 46, 2551-2564.

Lovell, S. C., Davis, I. W., Arendall, W. B. III, de Bakker, P. I. W., Word, J. M., Prisant, M. G., Richardson, J. S. \& Richardson, D. C. (2003). Structure validation by $\mathrm{C} \alpha$ geometry: $\phi, \psi$ and $\mathrm{C} \beta$ deviation. Proteins: Struct., Funct., Genet. 50, 437-450.

Lowry, O. H., Passonneau, J. V. \& Rock, M. K. (1961). Stability of pyridine nucleotides. J. Biol. Chem. 236, 2756-2759.

Lukatela, G., Krauss, N., Theis, K., Selmer, T., Gieselmann, V., von Figura, K. \& Saenger, W. (1998). Crystal structure of human arylsulfatase A: The aldehyde function and the metal ion at the active site suggest a novel mechanism for sulfate ester hydrolysis. Biochemistry 37, 3654-3664.

Madsen, D., Johansson, P. \& Kleywegt, G. J. (2002). Indonesia. $<$ http://xray.bmc.uu.se/dennis/>.

Main, P. (2006). Least-squares fitting of parameters. In Clegg, W. (Ed.). Crystal Structure analysis: Principles and practice. 2nd edition. Oxford: International Union of Crystallography/Oxford University Press, pp. 155-167.

Marino, T., Russo, N. \& Toscano, M. (2013). Catalytic mechanism of the arylsulfatase promiscuous enzyme from Pseudomonas aeruginosa. Chemistry 19, 2185-2192.

McCoy, A. J. (2004). Liking Likelihood. Acta Crystallogr. D60, 2169-2183.

McCoy, A. J., Grosse-Kunstleve, R. W., Adams, P. D., Winn, M. D., Storoni, L. C. \& Read, R. J. (2007). Phaser crystallographic software. J. Appl. Crystallogr. 40, 658-674. 
McKinney, J. D., zu Bentrup, K. H., Muñoz-Elías, E. J., Miczak, A., Chen, B., Chan, W., Swenson, D., Sacchettinik, J. C., Jacobs, W. R. Jr \& Russell, D. G. (2000). Persistence of Mycobacterium tuberculosis in macrophages and mice requires the glyoxylate shunt enzyme isocitrate lyase. Nature (London, U. K.) 406, 735-738.

Mesecar, A. D., Stoddard, B. L. \& Koshland, D. E. Jr (1997). Orbital steering in the catalytic power of enzymes: small structural changes with large catalytic consequences. Science 277, 202-206.

Meyer, A., Kossow, K., Gal, A., Steglich, C., Muhlhausen, C., Ullrich, K., Braulke, T. \& Muschol, N. (2008). The mutation p.Ser298Pro in the sulphamidase gene (SGSH) is associated with a slowly progressive clinical phenotype in mucopolysaccharidosis type IIIA (Sanfilippo A syndrome). Hum. Mutat. 29, 770.

Miyazaki, T., Masuda, N., Waragai, M., Motoyoshi, Y., Kurokawa, K. \& Yuasa, T. (2002). An adult Japanese Sanfilippo A patient with novel compound heterozygous S347F and D444G mutations in the sulphamidase gene. J. Neurol. Neurosurg. Psychiatry 73, 777-778.

Montfort, M., Vilageliu, L., Garcia-Giralt, N., Guidi, S., Coll, M. J., Chabás, A. \& Grinberg, D. (1998). Mutation 1091delC is highly prevalent in Spanish Sanfilippo syndrome type A patients. Hum. Mutat. 12, 274-279.

Müller, P., Köpke, S. \& Sheldrick, G. M. (2003). Is the bond-valence method able to identify metal atoms in protein structures? Acta Crystallogr. D59, 32-37.

Murshudov, G. N., Vagin, A. A. \& Dodson, E. J. (1997). Refinement of macromolecular structures by the maximum-likelihood method. Acta Crystallogr. D53, 240-255.

Murshudov, G. N., Skubák, P., Lebedev, A. A., Pannu, N. S., Steiner, R. A., Nicholls, R. A., Winn, M. D., Long, F. \& Vagin, A. A. (2011). REFMAC5 for the refinement of macromolecular crystal structures. Acta Crystallogr. D67, 355-367.

Muschol, N., Storch, S., Ballhausen, D., Beesley, C., Westermann, J. C., Gal, A., Ullrich, K., Hopwood, J. J., Winchester, B. \& Braulke, T. (2004). Transport, enzymatic activity, and stability of mutant sulfamidase (SGSH) identified in patients with mucopolysaccharidosis type III A. Hum. Mutat. 23, 559-566.

Muschol, N., Pohl, S., Meyer, A., Gal, A., Ullrich, K. \& Braulke, T. (2011). 
Residual activity and proteasomal degradation of p.Ser298Pro sulfamidase identified in patients with a mild clinical phenotype of Sanfilippo A syndrome. Am. J. Med. Genet. A 155, 1634-1639.

Myette, J. R., Soundararajan, V., Behr, J., Shriver, Z., Raman, R. \& Sasisekharan, R. (2009). Heparin/Heparan sulfate N-sulfamidase from Flavobacterium heparinum: structural and biochemical investigation of catalytic nitrogen-sulfur bond cleavage. J. Biol. Chem. 284, 35189-35200.

Nardini, M. \& Dijkstra, B. W. (1999). $\alpha / \beta$ hydrolase fold enzymes: the family keeps growing. Curr. Opin. Struct. Biol. 9, 732-737.

Navaza, J. (1994). AMoRe: an automated package for molecular replacement. Acta Crystallogr. A50, 157-163.

Neufeld, E. F. \& Muenzer, J. (2001). The mucopolysaccharidoses. In The metabolic and molecular bases of inherited disease. 8th edn. New York: McGraw Hill. pp. 3421-3452.

Ochiai, T., Fukunaga, N. \& Sasaki, S. (1979). Purification and some properties of two $\mathrm{NADP}^{+}$-specific isocitrate dehydrogenases from an obligately psychrophilic marine bacterium, Vibrio sp., strain ABE-1. J. Biochem. 86, 377-384.

Oppenheimer, N. J. (1987). Chemical stability and reactivity of pyridine nucleotide coenzymes. In Dolphin, D., Avramovic, O. \& Poulson, R. (Eds.). Pyridine Nucleotide Coenzymes, Part A. New York: Wiley. pp. 323-365.

Painter, J. \& Merritt, E. A. (2006). TLSMD web server for the generation of multi-group TLS models. J. Appl. Crystallogr. 39, 109-111.

Palatinus, L. \& van der Lee, A. (2008). Symmetry determination following structure solution in P1. J. Appl. Crystallogr. 41, 975-984.

Paschke, E. \& Kresse, H. (1979). Multiple Forms of 2-deoxy-D-glucoside-2sulphamate sulfohydrolase from human-placenta. Biochem. J. 181, 677-684.

Patterson, A.L. (1934). A Fourier series method for the determination of the components of interatomic distances in crystals. Phys. Rev. 46, 372-376.

Patterson, A.L. (1935). A direct method for the determination of the components of interatomic distances in crystals. Z. Kristallogr. A90, 517-542. 
Pei, J., Kim, B.-H. \& Grishin, N. V. (2008). PROMALS3D: a tool for multiple protein sequence and structure alignments. Nucleic Acids Res. 36, 2295-2300.

Peng, Y., Zhong, C., Huang, W. \& Ding, J. (2008). Structural studies of Saccharomyces cerevesiae mitochondrial NADP-dependent isocitrate dehydrogenase in different enzymatic states reveal substantial conformational changes during the catalytic reaction. Protein Sci. 17, 1542-1554.

Perkins, K. J., Byers, S., Yogalingam, G., Weber, B. \& Hopwood, J. J. (1999). Expression and characterization of wild type and mutant recombinant human sulfamidase: Implications for Sanfilippo (mucopolysaccharidosis IIIA) syndrome. $J$. Biol. Chem. 274, 37193-37199.

Piotrowska, E., Jakóbkiewicz-Banecka, J., Tylki-Szymańska, A., Czartoryska, B., Wegrzyn, A. \& Wegrzyn, G. (2009). Correlation between severity of mucopolysaccharidoses and combination of the residual enzyme activity and efficiency of glycosaminoglycan synthesis. Acta Paediatr. 98, 743-749.

Pollard, L. M., Jones, J. R. \& Wood, T. C. (2013). Molecular characterization of 355 mucopolysaccharidosis patients reveals 104 novel mutations. J. Inherit. Metab. Dis. 36, 179-187.

Ramakrishnan, C. \& Ramachandran, G. N. (1965). Stereochemical criteria for polypeptide and protein chain conformations. II. Allowed conformations for a pair of peptide units. Biophys. J. 5, 909-933.

Read, R.J. (1986). Improved Fourier coefficients for maps using phases from partial structures with errors. Acta Crystallogr. A42, 140-149.

Reinscheid, D. J., Eikmanns, B. J. \& Sahm, H. (1994). Characterization of the isocitrate lyase gene from Corynebacterium glutamicum and biochemical analysis of the enzyme. J. Bacteriol. 176, 3474-3483.

Rivera-Colón, Y., Schutsky, E. K., Kita, A. Z. \& Garman, S. C. (2012). The structure of human GALNS reveals the molecular basis for mucopolysaccharidosis IV A. J. Mol. Biol. 423, 736-751.

Rodríguez, D. D., Grosse, C., Himmel, S., González, C., de Ilarduya, I. M., Becker, S., Sheldrick, G. M. \& Usón, I. (2009). Crystallographic ab initio protein structure solution below atomic resolution. Nat. Methods 6, 651-653. 
Rodríguez, D., Sammito, M., Meindl, K., de Ilarduya, I. M., Potratz, M., Sheldrick, G. M. \& Usón, I. (2012). Practical structure solution with ARCIMBOLDO. Acta Crystallogr. D68, 336-343.

Rossmann, M. G. \& Blow, D. M. (1962). The detection of sub-units within the crystallogrpahic asymmetric unit. Acta Crystallogr. 15, 24-31.

Rossmann, M. G., Moras, D. \& Olsen, K. W. (1974). Chemical and biological evolution of a nucleotide-binding protein. Nature (London, U. K.) 250, 194-199.

Rossmann, M. G. (1990). The molecular replacement method. Acta Crystallogr. A46, 73-82.

Rossmann, M. G. (2001). Molecular Replacement: historical background. Acta Crystallogr. D57, 1360-1366.

Rupp, B. (2010). Biomolecular crystallography: Principles, practice, and application to structural biology. New York: Garland Science.

Sammito, M., Millán, C., Rodríguez, D. D., de Ilarduya, I. M., Meindl, K., De Marino, I., Petrillo, G., Buey, R. M., de Pereda, J. M., Zeth, K., Sheldrick, G. M. \& Usón, I. (2013). Exploiting tertiary structure through local folds for crystallographic phasing. Nat. Methods 10, 1099-1101.

Sanfilippo, S. J., Podosin, R., Langer, L. \& Good, R. A. (1963). Mental retardation associated with acid mucopolysacchariduria (heparitin sulfate type). $J$. Pediatr. (N. Y., NY, U. S.) 63, 837-838.

Schomaker, V. \& Trueblood, K. N. (1968). On rigid-body motion of molecules in crystals. Acta Crystallogr. B24, 63-76.

Scott, H. S., Blanch, L., Guo, X.-H., Freeman, C., Orsborn, A., Baker, E., Sutherland, G. R., Morris, C. P. \& Hopwood, J. J. (1995). Cloning of the sulphamidase gene and identification of mutations in Sanfilippo A syndrome. Nat. Genet. 11, 465-467.

Sheldrick, G. M. (1990). Phase annealing in SHELX-90: Direct methods for larger structures. Acta Crystallogr. A46, 467-473. 
Sheldrick, G. M. \& Gould, R. O. (1995). Structure solution by iterative peaklist optimization and tangent expansion in space group P1. Acta Crystallogr. B51, 423-431.

Sheldrick, G. M. (2002). Macromolecular phasing with SHELXE. Z. Kristallogr. 217, 644-650.

Sheldrick, G. M. (2008). A short history of SHELX. Acta Crystallogr. A64, 112-122.

Sheldrick, G. M. (2010). Experimental phasing with SHELXC/D/E: combining chain tracing with density modification. Acta Crystallogr. D66, 479-485.

Sheldrick, G. M. (2012). XPREP (program). Bruker AXS Inc., Madison, Wisconsin, USA.

Sheldrick, G. M. (2013). XPRO (program). University of Goettingen.

Sherwood, D. \& Cooper, J. (2011). Crystals, x-rays and proteins: comprehensive protein crystallography. Oxford: Oxford University Press, pp. 432-433.

Shiio, I. \& Ozaki, H. (1968). Concerted inhibition of isocitrate dehydrogenase by glyoxylate plus oxalacetate. J. Biochem. 64, 45-53.

Sidhu, N. S., Delbaere, L. T. J. \& Sheldrick, G. M. (2011). Structure of a highly $\mathrm{NADP}^{+}$-specific isocitrate dehydrogenase. Acta Crystallogr. D67, 856869.

Sidhu, N. S., Schreiber, K., Pröpper, K., Becker, S., Usón, I., Sheldrick, G. M., Gärtner, J., Krätzner, R. \& Steinfeld, R. (2014). Structure of sulfamidase provides insight into the molecular pathology of mucopolysaccharidosis IIIA. Acta Crystallogr. D70, 1321-1335.

Sivia, D. S. \& Skilling, J. (2006). Data analysis: a Bayesian tutorial. 2nd edition. Oxford: Oxford University Press.

Sly, W. S. \& Vogler, C. (2013). The final frontier: crossing the blood-brain barrier. EMBO Mol. Med. 5, 655-657.

Steen, I. H., Madsen, M. S., Birkeland, N. \& Lien, T. (1998). Purification and characterization of a monomeric isocitrate dehydrogenase from the sulfate- 
reducing bacterium Desulfobacter vibrioformis and demonstration of the presence of a monomeric enzyme in other bacteria. FEMS Microbiol. Lett. 160, 75-79.

Steinfeld, R., Steinke, H. B., Isbrandt, D., Kohlschütter, A. \& Gärtner, J. (2004). Mutations in classical late infantile neuronal ceroid lipofuscinosis disrupt transport of tripeptidyl-peptidase I to lysosomes. Hum. Mol. Genet. 13, 2483-2491.

Stenlund, P., Lindberg, M. J. \& Tibell, L. A. (2002). Structural requirements for high-affinity heparin binding: alanine scanning analysis of charged residues in the C-terminal domain of human extracellular superoxide dismutase. Biochemistry 41, 3168-3175.

Stoddard, B. L., Dean, A. \& Koshland, D. E. Jr (1993). Structure of isocitrate dehydrogenase with isocitrate, nicotinamide adenine dinucleotide phosphate, and calcium at 2.5 - $\AA$ resolution: a pseudo-Michaelis ternary complex. Biochemistry $32,9310-9316$.

Stoddard, B. L. \& Koshland, D. E. Jr (1993). Structure of isocitrate dehydrogenase with $\alpha$-ketoglutarate at 2.7 - $\AA$ resolution: conformational changes induced by decarboxylation of isocitrate. Biochemistry 32, 9317-9322.

Stoddard, B. L., Cohen, B. E., Brubaker, M., Mesecar, A. D. \& Koshland, D. E. Jr (1998). Millisecond Laue structures of an enzyme-product complex using photocaged substrate analogs. Nat. Struct. Biol. 5, 891-897.

Sundaralingam, M. (1969). Stereochemistry of nucleic acids and their constituents. Allowed and preferred conformations of nucleosides, nucleoside mono-, di-, tri-, tetraphosphates, nucleic acids and polynucleotides. Biopolymers 7, 821860 .

Taylor, A. B., Hu, G., Hart, P. J. \& McAlister-Henn, L. (2008). Allosteric motions in structures of yeast $\mathrm{NAD}^{+}$-specific isocitrate dehydrogenase. J. Biol. Chem. 283, 10872-10880.

Terwilliger, T. C. (2000). Maximum-likelihood density modification. Acta Crystallogr. D56, 965-972.

Terwilliger, T. C. (2004). Using prime-and-switch phasing to reduce model bias in molecular replacement. Acta Crystallogr. D60, 2144-2149. 
Thorn, A. \& Sheldrick, G. M. (2013). Extending molecular-replacement solutions with SHELXE. Acta Crystallogr. D69, 2251-2256.

Valstar, M. J., Ruijter, G. J. G., van Diggelen, O. P., Poorthuis, B. J. \& Wijburg, F. A. (2008). Sanfilippo syndrome: A mini-review. J. Inherit. Metab. Dis. $31,240-252$.

Valstar, M. J., Neijs, S., Bruggenwirth, H. T., Olmer, R., Ruijter, G. J., Wevers, R. A., van Diggelen, O. P., Poorthuis, B. J., Halley, D. J. \& Wijburg, F. A. (2010). Mucopolysaccharidosis Type IIIA: Clinical spectrum and genotypephenotype correlations. Ann. Neurol. 68, 876-887.

Van Hove, J. L. K., Wevers, R. A., Van Cleemput, J., Moerman, P., Sciot, R., Matthijs, G., Schollen, E., de Jong, J. G. N., Carey, W. F., Muller, V., Nicholls, C., Perkins, K. \& Hopwood, J. J. (2003). Late-onset visceral presentation with cardiomoypathy and without neurological symptoms of adult Sanfilippo A syndrome. Am. J. Med. Genet. 118A, 382-387.

van Loo, B., Jonas, S., Babtie, A. C., Benjdia, A., Berteau, O., Hyvönen, M. \& Hollfelder, F. (2010). An efficient, multiply promiscuous hydrolase in the alkaline phosphatase superfamily. Proc. Natl. Acad. Sci. USA 107, 2740-2745.

von Bülow, R., Schmidt, B., Dierks, T., von Figura, K. \& Usón, I. (2001). Crystal structure of an enzyme-substrate complex provides insight into the interaction between human arylsulfatase A and its substrates during catalysis. J. Mol. Biol. 305, 269-277.

Wang, Z., Brämer, C. \& Steinbüchel, A. (2003). Two phenotypically compensating isocitrate dehydrogenases in Ralstonia eutropha. FEMS Microbiol. Lett. 227, 9-16.

Weber, B., Guo, X.-H., Wraith, J. E., Cooper, A., Kleijer, W. J., Bunge, S. \& Hopwood, J. J. (1997). Novel mutations in Sanfilippo A syndrome: implications for enzyme function. Hum. Mol. Genet. 6, 1573-1579.

Weber, B., van de Kamp, J. J., Kleijer, W. J., Guo, X.-H., Blanch, L., van Diggelen, O. P., Wevers, R., Poorthuis, B. J. \& Hopwood, J. J. (1998). Identification of a common mutation $(\mathrm{R} 245 \mathrm{H})$ in Sanfilippo A patients from The Netherlands. J. Inherit. Metab. Dis. 21, 416-422.

Weiss, M. S. \& Hilgengeld, R. (1997). On the use of the merging $R$ factor as 
a quality indicator for X-ray data. J. Appl. Crystallogr. 30, 203-205.

Wicken, J. S., Chung, A. E. \& Franzen, J. S. (1972). Isocitrate dehydrogenase from Azotobacter vinelandii: order of substrate addition and product release. Biochemistry 11, 4766-4778.

Wierenga, R. K., De Maeyer, M. C. H. \& Hol, W. G. J. (1985). Interaction of pyrophosphate moieties with alpha-helixes in dinucleotide binding-proteins. Biochemistry 24, 1346-1357.

Winn, M. D., Isupov, M. N. \& Murshudov, G. N. (2001). Use of TLS parameters to model anisotropic displacements in macromolecular refinement. Acta Crystallogr. D57, 122-133.

Winn, M. D., Ballard, C. C., Cowtan, K. D., Dodson, E. J., Emsley, P., Evans, P. R., Keegan, R. M., Krissinel, E. B., Leslie, A. G. W., McCoy, A., McNicholas, S. J., Murshudov, G. N., Pannu, N. S., Potterton, E. A., Powell, H. R., Read, R. J., Vagin, A. \& Wilson, K. S. (2011). Overview of the CCP4 suite and current developments. Acta Crystallogr. D67, 235-242.

Xu, X., Zhao, J., Xu, Z., Peng, B., Huang, Q., Arnold, E. \& Ding, J. (2004). Structures of human cytosolic NADP-dependent isocitrate dehydrogenase reveal a novel self-regulatory mechanism of activity. J. Biol. Chem. 279, 33946-33957.

Yang, B., Zhong, C., Peng, Y., Lai, Z. \& Ding, J. (2010). Molecular mechanisms of "off-on switch" of activities of human IDH1 by tumor-associated mutation R132H. Cell Res. 20, 1188-1200.

Yasutake, Y., Watanabe, S., Yao, M., Takada, Y., Fukunaga, N. \& Tanaka, I. (2002). Structure of the monomeric isocitrate dehydrogenase: evidence of a protein momomerization by a domain duplication. Structure 10, 1637-1648.

Yasutake, Y., Watanabe, S., Yao, M., Takada, Y., Fukunaga, N. \& Tanaka, I. (2003). Crystal structure of the monomeric isocitrate dehydrogenase in the presence of $\mathrm{NADP}^{+}$: insight into the cofactor recognition, catalysis, and evolution. $J$. Biol. Chem. 278, 36897-36904.

Yogalingam, G. \& Hopwood, J. J. (2001). Molecular genetics of mucopolysaccharidosis type IIIA and IIIB: Diagnostic, clinical, and biological implications. Hum. Mutat. 18, 264-281. 
Zhang, K. Y. J., Cowtan, K. D. \& Main, P. (2012). Phase improvement by iterative density modification. Int. Tables Crystallogr. F, pp. 385-400.

Zhang, W. \& Huiping, S. (2008). Hum. Genet. 124, 323. Gene symbol: SGSH. Disease: Sanfilippo syndrome type A. Hum. Genet. 124, 323.

Zhang, B., Wang, B., Wang, P., Cao, Z., Huang, E., Hao, J., Dean, A. M. \& Zhu, G. (2009). Enzymatic characterization of a monomeric isocitrate dehydrogenase from Streptomyces lividans TK54. Biochimie 91, 1405-1410.

Zheng, J. \& Jia, Z. (2010). Structure of the bifunctional isocitrate dehydrogenase kinase/phosphatase. Nature (London, U. K.) 465, 961-965. 